\title{
The behavioural phenotype in two dynamic mutation disorders: fragile $X$ syndrome and myotonic dystrophy
}

Citation for published version (APA):

Steyaert, J. G. P. L. E. (2002). The behavioural phenotype in two dynamic mutation disorders: fragile $X$ syndrome and myotonic dystrophy. [Doctoral Thesis, Maastricht University]. Universiteit Maastricht. https://doi.org/10.26481/dis.20021121js

Document status and date:

Published: 01/01/2002

DOI:

10.26481/dis.20021121js

Document Version:

Publisher's PDF, also known as Version of record

\section{Please check the document version of this publication:}

- A submitted manuscript is the version of the article upon submission and before peer-review. There can be important differences between the submitted version and the official published version of record.

People interested in the research are advised to contact the author for the final version of the publication, or visit the DOI to the publisher's website.

- The final author version and the galley proof are versions of the publication after peer review.

- The final published version features the final layout of the paper including the volume, issue and page numbers.

Link to publication

\footnotetext{
General rights rights.

- You may freely distribute the URL identifying the publication in the public portal. please follow below link for the End User Agreement:

www.umlib.nl/taverne-license

Take down policy

If you believe that this document breaches copyright please contact us at:

repository@maastrichtuniversity.nl

providing details and we will investigate your claim.
}

Copyright and moral rights for the publications made accessible in the public portal are retained by the authors and/or other copyright owners and it is a condition of accessing publications that users recognise and abide by the legal requirements associated with these

- Users may download and print one copy of any publication from the public portal for the purpose of private study or research.

- You may not further distribute the material or use it for any profit-making activity or commercial gain

If the publication is distributed under the terms of Article $25 \mathrm{fa}$ of the Dutch Copyright Act, indicated by the "Taverne" license above, 
The behavioural phenotype in two dynamic mutation disorders:

Fragile X Syndrome and Myotonic Dystrophy 



\title{
The behavioural phenotype in two dynamic mutation disorders:
}

\section{Fragile X Syndrome and Myotonic Dystrophy}

\section{PROEFSCHRIFT}

\author{
ter verkrijging van de graad van doctor aan \\ de Universiteit Maastricht, \\ op gezag van de Rector Magnificus, \\ Prof. Dr. A.C. Nieuwenhuijzen Kruseman \\ volgens het besluit van het College van Decanen \\ in het openbaar te verdedigen op \\ donderdag 21 november 2002 om 16.00 uur.
}

door

Jean Steyaert 
Promotores:

Prof.dr. J.-P. Fryns

Prof.dr. J.P.M. Geraedts

Prof.dr. W.M.A. Verhoeven (Erasmus Universiteit Rotterdam)

Beoordelingscommissie:

Prof.dr. J.J. van Os (voorzitter)

Prof.dr. M. Danckaerts (Katholieke Universiteit Leuven)

Dr. C. E.M. de Die-Smulders

Prof.dr. G. Neri (Università Cattolica del Sacro Cuore, Roma)

Prof.dr. R. Vlietinck 
To the patients and their families. 



\section{Contents:}

Part 1. Introduction and literature review $\quad 9$

Chapter I Introduction 11

Chapter 2 Introductory concepts 15

Chapter 3 Psychiatric genetics: the case of single gene disorders 27

Chapter 4 Fragile X syndrome 43

Chapter 5 The behavioural phenotype in Myotonic Dystrophy 55

Part II. Studies in Fragile X Syndrome and in Myotonic Dystrophy 77

Chapter 6 Cognitive Profile in Adult, Normal Intelligent Female Fragile X Carriers 79

Chapter 7 Molecular-Intelligence Correlation in Young Fragile X Males With a Mild CGG Repeat Expansion in the FMRI-Gene

Chapter $8 \quad$ A distinct neurocognitive phenotype in female fragile $\mathrm{X}$ premutation carriers assessed with visual attention tasks.

Chapter 9 A study of the cognitive and psychological profile in 16 children with congenital or juvenile myotonic dystrophy

Chapter 10 Emotional and Behavioural profile and Child Psychiatric Diagnosis in the Childhood Type of Myotonic Dystrophy.

Part III. General Discussion $\quad 135$

Chapter $11 \quad$ Single gene disorders and behavioural phenotype 136

$\begin{array}{lll}\text { Chapter } 12 \text { Dynamic mutation disorders } & 139\end{array}$

Part IV. References\& Summaries $\quad 143$

$\begin{array}{ll}\text { References } & 145\end{array}$

$\begin{array}{ll}\text { Summary } & 161\end{array}$

$\begin{array}{ll}\text { Samenvatting } & 165\end{array}$

$\begin{array}{ll}\text { Dankwoord } & 169\end{array}$

$\begin{array}{ll}\text { Curriculum Vitae } & 170\end{array}$

Publicaties 

Part I. INTRODUCTION AND

LITERAT URE REVIEW 

Chapter 1 Introduction 


\section{General remarks}

The genetics or heredity of behaviour characteristics has puzzled manhood since ancient times. It became a part of our culture and popular knowledge, in expressions like "it's in the blood". Pseudo-scientific knowledge in this area led to well-known excesses in the 20th century. In the second half of the 20 th century more thorough scientific research in this field was performed. This led to the birth of a new science, behavioural genetics. Initially, scientific research in this field started from different sides. In quantitative genetics, behavioural scientists tried to tease apart the role of heredity in psychiatric disorders and in normal psychological characteristics, e.g. intelligence or personality traits. Molecular geneticists looked for genes that could underlie behavioural characteristics or disorders. Clinical geneticists wanted to understand the pathways between genetic diseases and the impact that some of them have on intelligence, development and other aspects of behaviour. The ultimate goal has always been to find treatment opportunities that might reverse negative effects of genes on behaviour. In recent years, the three disciplines have become much closer as shown in organisations and conferences like the International Society of Psychiatric Genetics [ISPG, 2002] and the Society for the Study of Behavioural Phenotypes [SSBP, 2002].

What the last 50 years of research have most clearly taught is the extreme complexity of the pathways between genes and behaviour [Rutter et al., 1999b]. While relatively simple organisms like Drosophila (fruit fly) may show quite straightforward associations between a gene mutation and a particular behaviour [Pflugfelder, 1998], this is rather the exception than the rule in much studied mammalians like mice and men. Another common observation is that the effect of identifiable genetic defects on behaviour varies widely. This is the phenomenon of phenotypical heterogeneity. Genetic heterogeneity on the other hand, implies that different genetic disorders may cause a similar developmental disorder. This is seen in non-specific mental retardation.

In the field of neuropsychiatric genetics, research has shown that heredity is important in autism, schizophrenia, bipolar disorder, Attention Deficit Hyperactivity Disorder and Tourette syndrome. Nevertheless, for none of these well-studied disorders a single gene defect has been found that could explain at least a substantial part of the cases. Different alternatives for the one-gene-one-disorder model have been proposed. A first model is that of the additive effect of different genes: several gene defects add up to cause the clinical disorder. Each of 
them may have a different weight in the pathogenesis of the phenotype. A second model is that separate gene defects may cause the disorder phenotype, independently from each other. According to a third model, some neuropsychiatric disorders may be associated with functional variants (polymorphisms) of particular genes, instead of being associated with gene defects. Theoretically, these polymorphic genes may act independently from each other, or combine and have additive effects. This model is supposed to cause the variation in normal behaviour traits, like intelligence and temperament traits. However, studies, which show associations between particular gene polymorphisms and behavioural traits or psychiatric disorders, also demonstrate that these associations generally only explain a modest part of the variation of the phenotype (for a review, see [McGue and Bouchard, 1998]).

Genetic causes that may affect behaviour can roughly be classified in four categories: (1) single gene defects; (2) defects or absence of multiple genes, as observed in (cryptic) chromosomal abnormalities; (3) variations in single genes (polymorphism); (4) combinations of polymorphism in multiple genes. The first two categories are more manageable to research, as in a number of cases the genes involved are known. Even then, the pathway between gene and behaviour has proven to be complex. It can be influenced by molecular mechanisms at the locus of the gene defect, by other genes, by the environment, and by stochastic effects. The simplest model one can study is the behavioural effect of identifiable molecular defects in single genes. The influence of other genes, environment and chance are left out. Even so, this model may in itself explain a wide variance in the phenotype of several diseases. We have no idea of the proportion of neuropsychiatric disorders that are caused by single gene disorders. Yet, they offer us a particular opportunity to understand one aspect of the variability in the phenotype of neuropsychiatric disorders.

\section{Aims of this thesis:}

The aim of our research has been to explore the behavioural effects of two single gene disorders: Fragile X Syndrome and Myotonic Dystrophy (Steinert's Disease). The causal mechanism in both diseases is an unstable trinucleotide repeat expansion, or dynamic mutation. Dynamic mutations are of particular interest. Firstly, several disorders of the central nervous system are caused by this recently discovered kind of mutation. Secondly, a dynamic mutation consists of a range of expansion lengths that each may affect the phenotype to a different degree, or even in a qualitatively different way. Thirdly, dynamic mutations have a measurable variance at the molecular level, which can be correlated with variance at the phenotypical level. 
The molecular mechanism in Fragile X Syndrome and Myotonic Dystrophy is at least partially understood. The most common clinical aspects of both diseases have been well described. We have focused on two issues: (1) some less well-known aspects of the behavioural phenotype of both diseases, and (2) how variations at the molecular level differentially affect the behavioural phenotype.

\section{Outline of this thesis}

Part I consists of reviews and theoretical considerations on the behavioural phenotype in single gene disorders, in particular in Fragile X Syndrome and Myotonic Dystrophy. In Chapter 2, we outline a number of concepts and findings that are essential to understand the subsequent research. Chapter III is an overview of possible causes of phenotypical variation in single gene disorder. Chapter IV and V are literature reviews on Fragile $\mathrm{X}$ syndrome and Myotonic Dystrophy, with special interest for the behavioural phenotype of these disorders.

Part II brings together a number of publications on the behavioural phenotype in Fragile X syndrome and Myotonic Dystrophy, studied by the research groups in Leuven and Maastricht in the period 1992-2002. The studies in Fragile X Syndrome describe (1) neurocognitive characteristics in female fragile $\mathrm{X}$ full mutation carriers of normal intelligence (Chapter 6), (2) neurocognitive characteristics in female fragile $\mathrm{X}$ premutation carriers (Chapter 8 ) and (3) the effect of incomplete hypermethylation of the fragile $\mathrm{X}$ full mutation in boys (Chapter 7). In the studies in Myotonic Dystrophy we focused on the behavioural phenotype of adolescents and young adults with the juvenile onset type of the disease, in a stage of the disease process where behavioural problems prove to be more disabling than the mild or almost absent muscle symptoms. This research highlights the effect of the Myotonic Dystrophy gene mutation on the central nervous system. Chapter 9 presents mainly the cognitive and behavioural characteristics in juvenile Myotonic Dystrophy. In Chapter 10 the findings on behaviour characteristics in childhood onset Myotonic Dystrophy are analysed further in a new group of subjects.

In Part III, conclusion remarks and comments are made.

\section{References:}

All references have been compiled at the end of the thesis 
Chapter 2 Introductory concepts 


\section{Behavioural phenotype}

\subsection{Definition}

When Langdon-Down first described Down's syndrome, he described particular behavioural characteristics like cheerfulness and musicality in the subjects with this syndrome (LangdonDown, 1866, cited in [O'Brien, 1992]). Nyhan was probably the first to use the term "behavioural phenotype" in the context of behaviour associated with genetic diseases in man, when he described the compulsive self-injurious behaviour that is consistently found in subjects with Lesch-Nyhan syndrome [Nyhan, 1972].

Since then, the term has acquired different meanings and interpretations. In their very restrictive definition, Flint and Yule require two criteria to apply the term "behavioural phenotype" to the association between a particular behaviour and a genetic disorder [Flint and Yule, 1994]. The first is that there must be "a distinctive behaviour that occurs in almost every case of the condition and rarely (if at all) in other conditions". The second criterion is that a genetic anomaly should be demonstrated, which has a direct relationship with the observed phenotype. Both criteria are met in a very limited number of genetic disorders, like Lesch-Nyhan syndrome. However, for the majority of genetic disorders in which more or less characteristic behavioural features have been described, the first condition will never be met. Hyperexcitability and several other behavioural characteristics are present in many but by far not all boys with the fragile-X syndrome. The same symptoms may occur in other subjects with a moderate mental retardation without the fragile- $X$ syndrome. Consequently, this symptom does not meet the first criterion of Flint and Yule. The second criterion, of a demonstrable genetic anomaly, is met for a growing number of genetic disorders, following advances in molecular biology. This definition of the term behavioural phenotype is obviously too narrow. It should be noted that a similar definition would also be of no use in the categorisation of somatic phenotypes, where variability in the phenotype is more often the rule than the exception.

At the other end of the spectrum of interpretations lie the too global ones. Complex behavioural or psychiatric syndromes, e.g. non-verbal learning disability or autism, have been described as "the" behavioural phenotype of a particular genetic disorder. More than a decade ago, it was claimed that fragile X syndrome was the underlying cause in up to $25 \%$ of children with autism [Gillberg and Wahlstrom, 1985]. This view was refuted in a metaanalysis including 5601 males with mental retardation and 1006 males with autism, all tested 
for fragile X [Fisch, 1992]. The study showed that the prevalence of autism in males with fragile $\mathrm{X}$ is not significantly higher than in males with the same level of mental retardation without fragile X. An important problem in this kind of errors is that statements on behavioural phenotypes are often made on small samples of subjects with a genetic syndrome [Flint, 1998]. This is partially understandable as the prevalence of many genetic syndromes is very low. Collaboration between several research centres can circumvent this problem. Another common error results from the way in which the behavioural phenotype is measured. Firstly, with the best intentions classical behaviour questionnaires or neuropsychological tests are used in groups of subjects with a genetic syndrome and mental retardation (MR). Children with MR have on average a threefold prevalence of behaviour problems compared with individuals with normal intelligence [Einfeld and Tonge, 1996]. Many neuropsychological tests correlate with intelligence. The use of instruments that have only been validated for a normal population will inevitably result in an abnormal prevalence of problems. Instruments that are appropriate for the cognitive level of the subjects and/or control groups should be used. Secondly, in some genetic syndromes, a subset of symptoms may be observed that are also observed in a particular behaviour disorder, while the other characteristics of this behaviour disorder are not present in subjects with the genetic syndrome under study. This may erroneously lead to the conclusion that the behavioural phenotype of the genetic disorder is a particular behaviour syndrome. Boys with fragile $\mathrm{X}$ have speech and language delays, show repetitive behaviours, signs of hyperexcitability, like toe-walking and hand-flapping, and abnormal eye contact. All these symptoms are often seen in autism [Reiss and Freund, 1992]. However, most subjects with fragile $\mathrm{X}$ have normal emotion recognition and social empathy, while deficiencies in these abilities are core problems in autism [Maes et al., 1993; Simon and Finucane, 1996]. Sometimes there is a stronger association between a genetic disorder and a complex behaviour syndrome: $25-50 \%$ of subjects with tuberous sclerosis have all the core symptoms of autism [Bolton P.F. and Griffiths, 1997; Smalley, 1998].

Finally, when using classical questionnaires, one may overlook some typical aspects of the behavioural phenotype. Many boys with fragile X syndrome bite their thumb root when excited, subjects with 17p-deletion (Smith-Magenis) syndrome show self-hugging and poikiloembolomania (the habit of inserting objects in body orifices), and subjects with PraderWilli syndrome often pick their skin. These rare symptoms are not items of the usual behaviour questionnaires. They require careful naturalistic observation of a number of subjects with a particular genetic syndrome in order to develop specific questionnaires, with 
which research on the behavioural phenotype of that syndrome can be conducted on a larger scale.

Somewhere between a too restrictive and a too global definition of the term behavioural phenotype, we can define the term behavioural phenotype of a genetic disorder as the range of behaviour characteristics that are significantly associated with the disorder. In fact, this approach of the behavioural phenotype is quite similar to that of the somatic phenotype. Which is not surprising, as in both cases gene defects are the underlying cause of the phenotype? The pathways between molecular defects and the resulting observed somatic or behavioural phenotype are different, or at least partially different. Indeed, there may be intertwinement of both at different levels. In Noonan syndrome, feeding problems are caused by early orofacial dyspraxia inducing phobic avoidance [OBrien, 1992]. In velo-cardio-facial syndrome, anomalies and dysfunction of the soft palate may underlie feeding problems in infancy [Swillen et al., 2000].

We will clarify three aspects of the proposed definition of the behavioural phenotype behaviour, range, and significantly associated.

In this context, the term behaviour should be used in its broadest sense and include: (1) specific and/or developmental delays, e.g. mental retardation, language development disorder; (2) personality characteristics and general dimensions of behaviour, e.g. amiable, shy, aggressive, hyperactive; (3) complex clusters of pathological behaviour like psychiatric syndromes, e.g. cycloid psychosis in Prader-Willi syndrome (PWS) [Verhoeven et al., 1998, 2002a]; (4) other characteristic behaviours that cannot be classified in a psychiatric syndrome and that may be syndrome-specific, e.g. gaze-avoidance in fragile $\mathrm{X}$ syndrome, skin-picking in Prader-Willi syndrome. Developmental delays, personality characteristics and behavioural dimensions should be assessed with psychometrical instruments; psychiatric syndromes with structured psychiatric interviews and standardised observation instruments; and unclassifiable symptoms by means of observation lists based on earlier naturalistic observation [Dykens, 1995].

The concept of a range of behaviours is delicate. It is based on what sound research teaches on the individual variability of behaviour in genetic syndromes. In a large group of individuals with a particular genetic disorder, statistical analysis and use of a control group may show that a number of behaviour characteristics are associated with the syndrome. Some subjects will display all these characteristics, while others will only partially exhibit the full spectrum. The studies on fragile $\mathrm{X}$ syndrome and myotonic dystrophy in this thesis are examples of this phenomenon. Other examples include studies on the behavioural phenotype 
of velo-cardio-facial syndrome (VCFS) [Swillen et al., 1999; Verhoeven et al., 2002b; Vogels et al., 2002]. In Chapter 3 we will review causes of this variability.

The aspect significantly associated means that the characteristics and boundaries of a particular behavioural phenotype have been obtained by quantitative and statistical analysis in sufficiently large groups of subjects, where possible with the use of control groups.

\subsection{Behavioural phenotype and psychiatric syndromes}

As we learned from the hapless story of the supposed association between autism and fragile $\mathrm{X}$ syndrome, a cautionary note is required when psychiatric syndromes are found to be part of a behavioural phenotype. Our knowledge of the pathophysiological mechanisms underlying psychiatric syndromes and the reasons why symptoms cluster together in such syndromes is still limited. Until we have a complete understanding of their causes, the observed clusters of symptoms remain syndromes and not diseases. Research on gene-behaviour relationships may even show that the currently accepted clustering into syndromes and the boundaries between them is rather arbitrary and should be re-evaluated in the future [Rutter et al., 1999b] (see also Chapter 3).

When a significant number of subjects with a particular genetic disorder display a cluster of symptoms that can be categorised as a psychiatric syndrome, we can say that this psychiatric syndrome is part of the behavioural phenotype. In such cases, the use of the term "psychopathological phenotype" is appropriate, as proposed by Verhoeven and co-workers [Verhoeven et al., 2000]. A major advantage is that affected individuals will receive the necessary medical and psychological attention for the psychiatric problems. However, a number of subjects with the genetic disorder may only display a number of the symptoms and thus not fulfil the criteria for the psychiatric syndrome. It is then cautionary not to see these symptoms as a pars pro toto and diagnose the psychiatric syndrome for the erroneous reason that this psychiatric syndrome was described as a part of the behavioural phenotype of that genetic disorder. Moreover, genetic disorders may be associated with a set of psychiatric symptoms that do not match well with existing categories. Consequently, different investigators may come to disparate diagnoses in the same genetic disorder, depending on their diagnostic perspectives [Verhoeven and Tuinier, 2001; Wang et al., 2000]. In this respect, the psychopathological profile of VCFS is rather unique and lacks key symptoms to meet the criteria for schizophrenia or for bipolar disorder and the term velo-cardio-facial psychiatric syndrome has been proposed [Verhoeven et al., 2000; Vogels et al., 2002]. 
Psychiatric syndromes observed in genetic syndromes are an epiphenomenon and developmental product of the underlying genetic defect. Probably, when genetic defects that are not associated with a known genetic disorder underlie a psychiatric syndrome, the types of mechanisms between gene and behaviour are not fundamentally different from those when a psychiatric syndrome is associated with genetic disorder and is a part of its phenotype: e.g. panic and phobic disorders had been known since long when it was discovered that a polymorphic genomic duplication on chromosome 15, not associated with distinct dysmorphic signs, is a susceptibility factor for these disorders [Gratacos et al., 2001].

\section{Mental retardation}

Mental retardation (MR) is probably the most common behavioural feature associated with genetic disorders affecting the central nervous system. The essential feature of MR is significant sub-average general intellectual functioning that is accompanied by significant limitations in adaptive functioning in several skill areas: communication, self-care, home living, social skills, functional academic skills, work, and other. The onset must occur before the age of 18 years, and generally much earlier [American Psychiatric Association, 1994]. General intellectual functioning is defined by intelligence quotient (IQ), obtained by assessment with standardized intelligence tests. Significant sub-average general intellectual functioning is defined as an IQ of at least two standard deviations below the average, which corresponds with an IQ of 70 or less. The presenting symptoms are generally impairments in adaptive functioning [American Psychiatric Association, 1994]. Adaptive functioning refers to the way in which individuals cope with normal life demands and how well they meet the standards of personal independence. Impairment in adaptive functioning can be measured with scales, like the Vineland Adaptive Behaviour Scales [Sparrow et al., 1984]. In some individuals, e.g. subjects with autism, there may be an important difference between intellectual functioning and adaptive behaviour [Schatz and Hamdan-Allen, 1995]. Both aspects should be taken into account when assessing an individual with developmental delays. Different degrees of mental retardation can be specified, ranging from mild to profound mental retardation, reflecting the level of intellectual impairment.

The prevalence of mental retardation is difficult to measure in large populations, but has declined in Western countries from several percent to approximately $0.6 \%-1 \%$ [Richardson, 1989; Stromme and Valvatne, 1998; Szymanski and King, 1999]. Better nutrition is considered to have contributed to this decline in prevalence. 
The causes of mental retardation are manifold: genetic causes, environmental causes like malnutrition or toxins. Genetic causes are thought to account for approximately one third of all detectable aetiologies [Szymanski and King, 1999]. The prevalence of detectable genetic causes increases with the severity of the MR. These genetic causes can be divided in different subgroups (see Table 1) [Chiurazzi, 2001a; Pronk et al., 1999].

Table 1 Genetic cause of MR and other neuropsychiatric developmental disorders.

\begin{tabular}{|c|c|c|}
\hline Category & Subcategory & $\begin{array}{l}\text { Examples, associated with mental retardation } \\
\text { or other developmental disorders. }\end{array}$ \\
\hline \multicolumn{3}{|l|}{$\begin{array}{l}\text { Chromosomal } \\
\text { abnormalities }\end{array}$} \\
\hline \multirow[t]{2}{*}{ Aneuploidies } & Autosomes & Trisomy 21/Down syndrome \\
\hline & Sex-chromosomes & $\begin{array}{l}\text { 47,XXY-Klinefelter syndrome; } 45, X \text {-Turner } \\
\text { syndrome }\end{array}$ \\
\hline Mosaicism & & Tetrasomy $12 \mathrm{p}$-Pallister-Killian syndrome \\
\hline \multirow{3}{*}{$\begin{array}{r}\text { Deletions, duplications, } \\
\text { and re-arrangements }\end{array}$} & $\begin{array}{l}\text { Partial trisomies \& } \\
\text { duplications }\end{array}$ & $\begin{array}{l}\text { Distal 3p trisomy; inverted } 8 \mathrm{p} \text { duplication } \\
\text { syndrome }\end{array}$ \\
\hline & $\begin{array}{l}\text { Deletions \& } \\
\text { microdeletions }\end{array}$ & del $(8 p)$; del (22q)-velo-cardio-facial syndrome \\
\hline & $\begin{array}{l}\text { Cryptic translocations / } \\
\text { deletions / duplications }\end{array}$ & $\begin{array}{l}\text { Familial cases of MR with multiple congenital } \\
\text { anomalies }\end{array}$ \\
\hline \multicolumn{3}{|l|}{ Single gene disorders } \\
\hline & Autosomal recessive & Phenylketonuria; essential microcephaly \\
\hline & Autosomal dominant & $\begin{array}{l}\text { Neurofibromatosis type 1, SPCHI-gene mutation } \\
\text { with dysarthry, myotonic dystrophy }\end{array}$ \\
\hline & $\mathrm{X}$-linked & $\begin{array}{l}\text { Fragile X syndrome; diverse syndromic and } \\
\text { non-syndromic forms of X-linked MR }\end{array}$ \\
\hline & mitochondrial & Myoclonic epilepsy with ragged-red fibbers. \\
\hline Polygenic inheritance & & Non-specific familial MR \\
\hline
\end{tabular}

MR with a genetic origin can be subdivided according to the associated physical, neurological and biological signs (see Table 2).

In specific or syndromal MR, clinical, biochemical or other phenotypical findings are associated with MR. The MR is then part of a broader phenotype. Some genetic anomalies are associated with multiple congenital anomalies (MCA), among which facial and other dysmorphic signs. This is the case in e.g. Down syndrome and velo-cardio-facial syndrome. In phenylketonuria and other metabolic disorders, the MR is associated with biochemical 
findings, without visible clinical signs. In MASA-syndrome [Schrander-Stumpel et al., 1995; Weller and Gartner, 2001], Rett syndrome [Amir et al., 1999] and X-linked infantile spasms [Claes et al., 1997; Rugtveit, 1986], MR is associated with characteristic neurological signs. This subdivision is somewhat arbitrary, however, as some genetic syndromes present with signs from different subcategories. In Prader-Willi syndrome and Down syndrome, MR is associated with MCA's and a neurological symptom, muscle hypotonia.

In non-specific or non-syndromal MR, the MR is the only clinical finding and there are no dysmorphic or other clinical signs. It is often thought that non-specific MR is either caused by non-genetic factors, or, if genetic, by polygenic inheritance [Plomin et al., 2000; Rutter et al., 1996]. In a number of families with non-specific MR, a monogenic heredity has been found, and the underlying gene defect could be identified. On the X-chromosome, at least 33 such genes have already been cloned [Chiurazzi et al., 2001]. Autosomal forms of non-specific MR, like 3p25-ter syndrome [Higgins et al., 2000], seem more seldom than X-linked MR (XLMR) or are more difficult to identify.

Table 2 Clinical classifications of MR with a genetic origin, and a few examples.

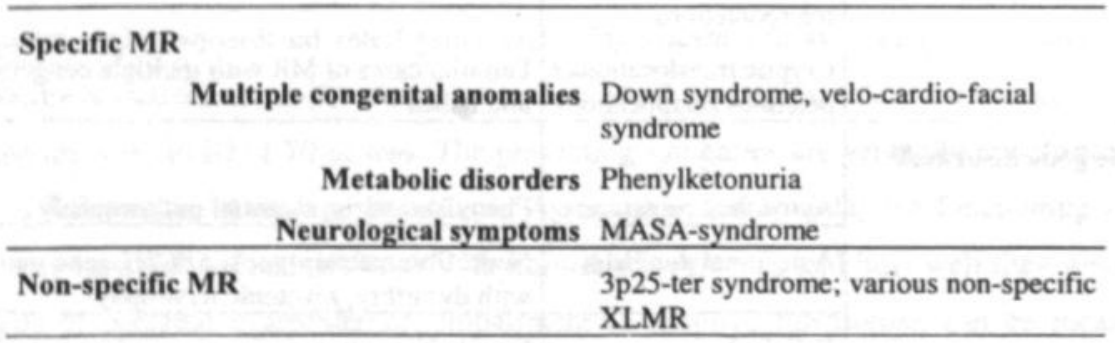

The classification in specific and non-specific MR is also ambiguous: more in-depth analysis of the phenotype of some so-called non-specific genetic causes of MR may eventually show that more subtle phenotypical signs are associated with demonstrable causes of isolated MR, but not with others. Such phenotypical signs may be specific neurocognitive deficits or other neuropsychiatric symptoms. It has been suggested that MR due to a mutation in a gene of the IL-receptor family located at $\mathrm{Xq} 22$, which is highly expressed in the hippocampus, might be associated with specific problems of short-term memory [Carrie et al., 1999]. Findings like this underscore the importance of thorough analysis and classification of not only the somatic phenotype, but also of the behavioural phenotype. 


\section{Other developmental disorders}

Developmental disorders are chronic conditions where there is a disturbance in the acquisition of cognition, language, motor, or social skills [Harris, 1998a]. Beside mental retardation, these include a variety of neuropsychiatric conditions: e.g. autism, language developmental disorder, motor coordination developmental disorder, and dyslexia. The taxonomy of these developmental disorders is based on the observed phenotype, and not on causal mechanisms [American Psychiatric Association, 1994]. During the last three decades, there has been an increasing interest in the causal mechanisms of these disorders, as testified in textbooks on developmental neuropsychiatry [Harris, 1998b] and psychopathology [Cicchetti and Cohen, 1995]. The role of genetics in the aetiology of developmental disorders is becoming increasingly clear [Rutter et al., 1990; Rutter, 1994; Rutter et al., 1999a].

Basically, the same broad classification of causes can be made as for mental retardation. Syndromal forms of autism exist. The autistic behaviour is then part of a broader clinical phenotype that is associated with a genetic defect. Various chromosomal defects have been found in individuals with autism [Gillberg, 1998]. The autism phenotype is also associated with some monogenic disorders, e.g. tuberous sclerosis [Smalley, 1998]. Non-syndromal autism can be caused by monogenic inheritance, as has been suggested in a number of families with multiple probands [Jamain et al., 2002]. In other subjects, a polygenic inheritance most probably underlies the disorder [Bolton P. et al., 1994]. A similar classification can be applied to the findings in other neuropsychiatric developmental disorders. However, the relative importance of the different causes of these developmental disorders is unknown and might be different from one disorder to the other.

\section{Dynamic mutations}

The term "dynamic mutation" was introduced to distinguish the unique properties of expanding, unstable DNA repeat sequences from other types of mutation [Richards R.I., 2001] (see also Chapter 3, section 5). In 1991, this mechanism was discovered in fragile $X$ syndrome [Verkerk et al., 1991] and spino-bulbar muscular atrophy [La Spada et al., 1991]. In 1992, a similar mechanism was found in myotonic dystrophy [Aslanidis et al., 1992; Harley et al., 1992a]. So far, at least 13 different dynamic mutations have been described, all affecting the central nervous system [Richards R.I., 2001]. Amongst these are Huntington's chorea, Friedreich ataxia, and a rare form of major psychosis associated with a dynamic mutation in the $S C A 8$ gene [Vincent J.B. et al., 2000]. 
Repeat sequences of two, three, four and sometimes more base pairs are common in the genome. They are generally stable in the population. For unclear reasons these repeat sequence sometimes become unstable and expand. The mechanism of the expansion is far from being elucidated. Trinucleotide repeats, in particular the pairs CCG/CGG and CAG/CTG are particularly prone to this phenomenon. Instability can occur from one generation to another (gametic instability), and/or within the same individual (somatic instability). In myotonic dystrophy both forms of instability occur, while in Huntington's chorea only gametic instability has been observed. Instability can consist of an increase of the number of repeats e.g. $(\mathrm{CGG}) \mathrm{n} \rightarrow(\mathrm{CGG}) \mathrm{n}+\mathrm{m}$, as well as a decrease, e.g. (CTG)n $\rightarrow(\mathrm{CTG}) \mathrm{n}-\mathrm{m}$. An increase (amplification) is more frequently observed. Repeat number and composition of the repeat (perfect repeat of the same trinucleotide, versus interruptions with other trinucleotides) influence instability of the repeat sequence. In general, alleles with higher repeat numbers and alleles that are free of interruptions are more unstable than those with a lower copy number and/or that contain interruptions [Richards R.I., 2001]. In some dynamic mutations, there is a parental gender bias in the amplification of the trinucleotide repeat from one generation to the next. In fragile $X$ syndrome, the repeat number is stable in paternal inheritance, and often unstable in maternal inheritance. In Huntington's chorea paternal inheritance on average leads to larger expansions than maternal inheritance [Norremolle et al., 1995].

Dynamic mutations are distributed across a range of relatively stable repeat lengths in the normal population. Beyond a certain repeat number, the repeat becomes unstable and is then associated with pathology in most of these diseases. This is then called a mutation. The relationship between repeat length and phenotype varies from disease to disease. (1) There may be a gradual correlation between repeat number and disease onset and/or severity. In Huntington's chorea the disease will develop in individuals who have more then 37 CAG repeats at the mutation site, and longer repeats are associated with earlier disease onset [Duyao et al., 1993]. (2) In some other dynamic mutation diseases, there is a bimodal distribution of the phenotype. In fragile X syndrome, expansions between 55 and 200 CGG repeats are unstable while subjects have a mostly normal phenotype. This is the fragile $\mathrm{X}$ premutation. The fragile $\mathrm{X}$ full mutation consists of amplifications of more than 200 repeats and is associated with the classical fragile $\mathrm{X}$ phenotype. In the $S C A 8$-gene expansions between 107-127 CTG repeats are associated with spinocerebellar ataxia, while expansions of more than 600 repeats are associated with major psychosis [Vincent J.B. et al., 2000].

Several pathogenic pathways between gene and phenotype have been described in dynamic mutations [Richards R.I., 2001]. In fragile X syndrome (see Chapter 4) and Friedreich's 
ataxia, the full mutation is associated with loss of gene expression and consequent lack of protein production. In myotonic dystrophy (see Chapter 5) there is evidence supporting an effect of the mutation on the functioning of other genes [Tapscott and Thornton, 2001]. Increased messenger RNA production has been suggested as a pathogenic mechanism in the fragile X premutation [Tassone et al., 2000c].

Once a trinucleotide repeat has reached a critical length and becomes unstable in one individual, it will remain unstable in the consequent generations, thus creating a "founder" effect [Laberge, 1989].

\section{X-linked mental retardation}

At the end of the 19th century, it was suggested that the prevalence of mental retardation is higher in males than in females [Johnson G.E., 1897]. The finding was confirmed in a survey of institutionalised mentally retarded persons in the U.K. [Penrose, 1938]. Lehrke [Lehrke, 1974] noted that this difference might be due to the absence of a second X-chromosome in males. Genes on the X-chromosome affecting brain development or functioning could explain why males were affected, while females could benefit from the homologue gene on the other X-chromosome. Years earlier, Martin and Bell had described sex-linked inheritance of mental retardation in a pedigree [Martin and Bell, 1943]. In this family, several males had moderate mental retardation, while females were not or only mildly affected. In 1969, Lubs described a fragile site on the long arm of the $\mathrm{X}$ chromosome, the marker $\mathrm{X}$ chromosome, to be associated with sex-linked mental retardation [Lubs, 1969]. Using improved cytogenetic procedures, Sutherland [Sutherland, 1978] demonstrated that the fragile site on the X-chromosome is present in only a number of families with X-linked mental retardation (XLMR). This helped to identify families with fragile-X syndrome (FXS) and to differentiate them from families with other forms of X-linked mental retardation [Howard-Peebles et al., 1979; HowardPeebles, 1982]. At re-examination, Martin and Bell's original pedigree proved to carry the fragile X chromosome [Richards B.W. et al., 1981].

Differentiating FXS from other forms of XLMR helped to specify the FXS phenotype [Fryns J. P., 1984; Fryns J. P. et al., 1984a; Hagerman R.J. et al., 1983]. The identification of FXS was an important step in the acceptance that $\mathrm{X}$-linked genes are responsible for at least a part of the excess of males in MR. Other X-linked syndromes associated with mental retardation have been delineated since [Chelly and Mandel, 2001].

XLMR can be associated with particular phenotypical characteristics other than the mental retardation. These are called specific or syndromic XLMR (MRXS). E.g., Rett syndrome is 
associated with a MECP2 gene mutation, X-linked hydrocephalus/MASA syndrome with ar LICAM-gene mutation, and Lesch-Nyhan disease with an HPRT-gene mutation. Other type: of XLMR are not associated with specific phenotypical signs and are called non-specific on non-syndromic XLMR (MRX). Examples of MRX are: the Oligophrenin-I gene, and the FMR2/FRAXE gene mutation [Chelly and Mandel, 2001]. A recent count of MRXS and MRX showed that 209 clinical forms of XLMR have been delineated [Chiurazzi, 2001b; Chiurazz et al., 2001; Frints et al., 2002; Schwartz and Stevenson, 2002]. A hundred and eighteen o these have been mapped, and 34 genes have been cloned.

Table 3 Total counts of XLMR, specific (MRXS), and non-specific (MRX).

\begin{tabular}{llccc}
\hline & & Total count & Mapped & Cloned \\
\hline MRXS & & & & \\
Recressive disorders. & & & & \\
& $\begin{array}{l}\text { MCA* } \\
\text { Syndromes }\end{array}$ & 81 & 36 & 7 \\
& Neuromuscular & 39 & 20 & 6 \\
& Metabolic & 12 & 2 & 10 \\
Dominant disorders & & 8 & 3 & 4 \\
Total disorders & & 140 & 61 & 27 \\
\hline MRX & & 69 & 57 & 7 \\
\hline Total entries & & 209 & 118 & 34 \\
\hline
\end{tabular}

$\bullet M C A=$ Multiple Congenital Anomalies. 


\section{Chapter 3 Psychiatric genetics: the case of single gene disorders}

Jean Steyaert ${ }^{1,2}$, Jean-Pierre Fryns ${ }^{3}$ (2002).

' Centre for Clinical Genetics, University of Maastricht, the Netherlands

2 Department of Child Psychiatry, University of Leuven, Belgium

${ }^{3}$ Center for Human Genetics, University of Leuven, Belgium

Review in: European Child and Adolescent Psychiatry. In Press. 


\section{Abstract}

Quantitative and molecular genetics have made important developments in the last three decades. There is increasing evidence of the role of heredity in the field of neuropsychiatric disorders in children. Sofar, only a few pathways between genes and behaviour have been unravelled.

Quantitative genetics puts polygenic inheritance models forward. Molecular genetic research based on these models seems promising, but until now has provided only a limited explanation for the variance in the studied neuropsychiatric disorders. In these models, the complexity of the expression of a single gene grows exponentially with the number of genes involved. Consequently, research on the gene-phenotype relationships and phenotypical variability in such models is extremely complex.

The candidate gene approach, in which the gene-phenotype pathway of a single gene is studied, is more manageable, and in our opinion essential in understanding multiple gene models. We discuss recent findings in the field and their relevance for neuropsychiatric phenotypes. Single gene defects will only explain a part of the range of neuropsychiatric disorders in children, but the evidence that this approach can generate may help to clarify neuropsychiatric phenotypes. The discovery of single gene disorders in subgroups of subjects with a neuropsychiatric phenotype may result in new perspectives for their treatment. 


\section{Introduction}

Our cultural ancestors had clear ideas about the heredity of behaviour and personality characteristics. Homer attributed to Telemachos the wisdom and godlikeness of his famous father Odysseus [Homer, 1995]. Telemachos however, had never met his father during the first twenty years of his life. Nature was stronger than nurture at that time. Much later Sir Francis Galton [Plomin et al., 2000] approached this phenomenon in a more scientific way by studying the hereditability of cognitive abilities. This was the basis of quantitative genetics. Ever since, and in particular in the past three decades, the heredity of behavioural traits and psychiatric conditions has been widely studied [Gelernter, 1999; Plomin et al., 2000; Rutter et al., 1999b]. This has not only led to interesting findings on the heredity of personality characteristics, cognitive abilities and psychiatric conditions, but also to more accurate descriptions of shared and non-shared environmental effects on development [McGue and Bouchard, 1998; Rutter et al., 1999b]. In adult psychiatry, the heredity of schizophrenia and bipolar disorder has been studied extensively [Nurnberger et al., 1994]. In developmental psychiatry, autism has been a particular focus of research [Rutter, 2000]. This has resulted in valuable information on the heredity and the phenotype of autism, e.g. the concept of a broader phenotype in some non-autistic family members of autistic probands [Le Couteur et al., 1996; Murphy M. et al., 2000]. The development of statistical techniques [Rutter et al., 1999b] has allowed the study of a range of questions like hypotheses about possible mechanisms involved in sex differences, or the number of genes that might play a role in the susceptibility for a child psychiatric disorder.

Several methods can be applied in the search for genes affecting behaviour and/or mental disorder (see Table 4 for an overview). We refer to other articles on this subject for a more comprehensive review of how these methods can be applied in neuropsychiatric research [Rutter et al., 1999b]. Family and genetic epidemiology studies have been accompanied by molecular genetic studies, in search for genes affecting behaviour and psychiatric disorders. Initially, linkage analysis has been used to look for single genes that could cause the heredity of neuropsychiatric disorders. Up to now, successes like the discovery of the gene for Huntington's disease [Gusella, 1984], in which only one gene (defect) is necessary and sufficient for a particular disease phenotype, have not been reproduced in psychiatric conditions. This has different reasons.

Firstly, research suggests that neuropsychiatric disorders are often caused by several interacting genetic loci, or quantitative trait loci (QTL's), rather than by a single major locus. 
E.g., this has been demonstrated in schizophrenia [Risch, 1990], and in autism. In autism the concordance in monozygotic twins is more than twice that in dizygotic twins, suggesting that more than one gene is involved in the heredity of the disorder in these cases [Rutter et al., 1999b]. Linkage analysis searching for such QTL's in autism has shown that probably more than 15 loci are involved [Risch et al., 1999], while not a single gene at these loci has been identified yet. Though there are robust arguments that the phenomenon of QTL's operates in a substantial number of cases, it is a particularly complex field of research, in part because we do not exactly know what to look for. This obstacle can be alleviated by looking in the other direction: instead of going backward and look for QTL's starting from the phenotype, one can focus on the association of candidate genes with a particular neuropsychiatric disorder [Evans et al., 2001]. This has been performed e.g. in Attention Deficit Hyperactivity Disorder (ADHD) [Comings et al., 2000]. However, a problem that remains in a forward genetic approach is that it is not clear how different genes combine to cause a particular neuropsychiatric phenotype. This is not necessarily through several defective genes, but also through particular combinations of polymorphic genes. In the population, different alleles of one gene co-exist. These polymorphisms lead to small differences in the functioning of the gene product. When several such genes are involved, particular combinations may lead to dysfunction, as proposed in the study on the additive effects of polymorphisms of three genes involved in dopamine function in Tourette syndrome [Comings et al., 1996]. Secondly, different and independent genetic errors can cause the same psychiatric disorder. Different chromosomal abnormalities are independently associated with autism [O'Brien and Yule, 1995]. This phenomenon demonstrates the genetic heterogeneity of neuropsychiatric disorders. It is a limitation in linkage analysis based on a QTL model. Such research requires large groups of subjects or families, and these groups are likely to be genetically heterogeneous. In different families, completely independent genes or combinations of genes may cause a similar neuropsychiatric phenotype, blurring linkage analysis. Thirdly, a psychiatric phenotype and the underlying genetic abnormalities may not always coincide [Rutter et al., 1999b]. The same genetic liability seems to underlie conduct disorder and oppositional defiant disorder, which are generally considered as different diagnostic categories. A genetically influenced behaviour trait may be a risk factor for a psychiatric disorder, independently of the genetic liability for the disorder itself. This was demonstrated for the genetically influenced personality trait neuroticism as a risk factor for depressive disorder [Kendler, 1996]. A fourth reason is that, ideally, the definition of a particular disease phenotype should precede the search for causative genes. On the other hand, it often only 
becomes apparent which phenotype is associated with a particular genetic defect or variant once the latter has been identified. Only after it became possible to detect the fragile- $\mathrm{X}$ site $\mathrm{Xq} 27.3$, it became clear that not only males, but also carrier females often have clinical characteristics of the fragile-X syndrome [Fryns J. P., 1984; Hagerman R.J. et al., 1986a]. This paradox complicates research on gene-behaviour relationships when starting from the psychiatric phenotype.

\section{Table 4: Brief overview of main strategies in molecular research for genes affecting} behaviour or mental disorders

\begin{tabular}{|c|c|c|}
\hline Searching for $\rightarrow$ & Single genes & Multiple genes \\
\hline $\begin{array}{l}\text { From behaviour } \\
\text { to genes: }\end{array}$ & $\begin{array}{l}+ \text { Relative routine for linkage } \\
\text { analysis. If single locus exists, there } \\
\text { is a good chance of finding it. }\end{array}$ & $\begin{array}{l}\text { This is most likely closer to the } \\
\text { reality of the genetics of } \\
\text { neuropsychiatric disorders. }\end{array}$ \\
\hline $\begin{array}{l}\text { Backward } \\
\text { approach through } \\
\text { linkage analysis }\end{array}$ & $\begin{array}{l}\text { Good indication when there is a } \\
\text { clear disease phenotype with } \\
\text { monogenic inheritance in a large } \\
\text { pedigree. }\end{array}$ & $\begin{array}{l}\text { + QTL models can be taken into } \\
\text { account } \\
\text { - Long way from finding multiple } \\
\text { loci to finding genes. }\end{array}$ \\
\hline & $\begin{array}{l}\text { - Long way from finding locus to } \\
\text { finding gene. } \\
\text { - No results if no single major gene }\end{array}$ & $\begin{array}{l}\text { - Even if genes are found, there is a } \\
\text { good chance that their pathogenetic } \\
\text { mechanism is unknown. }\end{array}$ \\
\hline & $\begin{array}{l}\text { effect, or if penetrance of major } \\
\text { gene is low. } \\
\text { - Finding a single major gene will }\end{array}$ & $\begin{array}{l}\text { - At molecular level, understanding } \\
\text { the simultaneous effect of multiple } \\
\text { genes is extremely complex. }\end{array}$ \\
\hline
\end{tabular}
often only reflect part of reality in complex disorders.

+ Effect of one gene on the phenotype can be studied in depth.

\section{From genes to} phenotype:

Forward approach through association / candidate gene studies

+ Mechanisms of expression and variability can be studied for that gene, as well as function of the gene product.

+ The procedure may point to other related candidate genes.

- Inherently, this approach does not allow describing combined and quantitative effects of sets of genes.

- Association studies with genes with an unknown function may lead to false positive results if there is another reason for association between the gene and the phenotype (e.g. ethnic group...)
+ Not only individual effect, but also additive effect and interactions of several (candidate) genes on a phenotype can be studied.

+ QTL models can be studied.

- What happens at the molecular level is difficult to study.

- Small or non-significant effect of candidate genes in a complex model may erroneously lead to removing these genes from the model.

- Inherent multiple analysis may lead to erroneously significant effects, which do not reflect an effect at the molecular level.

\footnotetext{
$"+"$-signs indicate advantages of the approach, and "-"-signs limitations. The list of advantages and limitations is not exhaustive. $Q T L=$ Quantitative Trait Loci.
} 
Nevertheless, in some neuropsychiatric disorders, it has been possible to define a phenotype that could be linked to a causative gene. In a single family aggressive behaviour and borderline intellectual functioning were found to be associated with a mutation in the MAO-A gene [Brunner et al., 1993]. In the KE-family, a specific language impairment is associated with the FOXP2 gene [Hurst et al., 1990; Lai et al., 2001]. However, even in a seemingly onegene-one-behaviour situation like Brunner's syndrome, it became clear that the relationship between the gene defect and the behaviour is not so straightforward. The aggressive behaviour of the subjects with a $M A O-A$ gene mutation may be due to distant effects of the gene on the metabolism of other neurotransmitters [Brunner, 1996].

In conclusion, linkage analysis looking for single causative genes in neuropsychiatric disorders has only led to very modest results. Linkage analysis looking for multiple genes is somehow like looking for unknown objects in a haystack. Sometimes this may be a successful endeavour, but it is generally more rewarding to know more about the kind of object one is searching. This consideration is in favour of a forward approach based on multiple (candidate) genes. However, this does not decrease the complexity of the problem. Considering the given arguments and questions, we advocate a forward single gene approach as preliminary step towards more complex models with multiple candidate genes. Understanding the variability and mechanisms influencing the expression of single genes is in our view elementary in dealing with multiple gene models, just like a mason should be acquainted with bricks and mortar before building houses. Moreover, in families where a single gene has a major effect on the occurrence of a neuropsychiatric disorder, it may help to understand why there such variability in the phenotype remains.

\section{Aim of this review}

Variability in phenotypic expression is rather the rule than the exception in single gene disorders, e.g. Fragile X syndrome or neurofibromatosis typel. A similar variability may be expected for genes that have an effect in the pathogenesis of a particular neuropsychiatric disorder. We reviewed a number of the mechanisms that cause variability, to gain a better understanding of the difficulties occurring in our research with single candidate genes in neuropsychiatric disorders, in particular mental retardation and autism. These difficulties are at least multiplied in similar research with multiple candidate genes. We publish this review, as it may help to understand why for many years to come child psychiatrists will have to deal with a discrepancy between general knowledge about the hereditability of neuropsychiatric disorders and what can actually be demonstrated in an affected individual or family. 


\section{Methods}

Literature on gene/behaviour research was selected from more than 6000 Medline references on the topic. One group of articles covers the past four decades, and consists mainly of review articles by influential authors, and articles that have been seminal in the field of gene/behaviour research. The second group of selected articles are specific research findings on molecular mechanisms in single gene disorders. For obvious reasons, we favoured papers on single gene disorders with an associated behavioural phenotype, though this was not a strict limitation. Another bias was that we favoured papers on psychiatric and genetic conditions that are subject of research in our centre, e.g. autism, mental retardation, dynamic mutations, Velo-Cardio-Facial Syndrome (VCFS).

\section{Gene/behaviour relationships in single-gene disorders with a behavioural phenotype}

Behaviour researchers often assume that single gene disorders have a dichotomous effect on development, with a clear-cut phenotype when the gene defect is present versus normality when it is not [Plomin et al., 2000]. While this may be true in Huntington's chorea, the dichotomy between normality and impairment is not always so clear in other conditions. In Neurofibromatosis type 1 (NF1), even when pathognomonic physical signs are present, the behaviour of the subject may not be affected, while other subjects manifest marked learning problems and/or behavioural impairment like poor social abilities and poor impulse control. In a family, some affected members may only show minor physical signs, while others, with exactly the same $N F 1$ gene mutation have marked behavioural impairment [Kayl and Moore, 2000]. The same phenomenon occurs in females with the fragile $X$ full mutation, of which at least $25 \%$ have no cognitive or behavioural impairment. It is also seen in VCFS caused by a microdeletion of a small group of genes on chromosome 22q11 (22q11-deletion). Some of these subjects have marked cognitive impairment, a higher than expected number of them has psychotic disorders, but others have no marked behavioural impairment. This phenomenon is independent of cardiac malformations that also occur with great variability in VCFS [Swillen et al., 1999]. In tuberous sclerosis, some subjects have severe autism and/or epilepsy, while others are normal [Hunt and Shepherd, 1993].

These examples demonstrate that disorders of single genes that are expressed in the central nervous system, do not necessarily have a dichotomous effect on behaviour, but rather lead to 
a spectrum of both quantitative and qualitative impairment. In some single gene disorders, the impairment will be severe and look like a dichotomy. Only a small minority of fragile-X males have a cognitive level in the normal range [Steyaert et al., 1996], while the majority have moderate mental retardation. In other single gene disorders, e.g. NF1, this dichotomy is not present and the phenotype is a continuum ranging from impairment to normality. These genes have a reduced penetrance in a number of individuals, and these carriers transmit the disease through their pedigree while they are clinically unaffected. Thus, in a number of families with several members with a neuropsychiatric disorder, a single gene anomaly can be associated with a distinct phenotype, while in other families the same phenotype is caused by additive effect of multiple genes. For example, in a sample of subjects with schizophrenia, a small but higher than expected number have 22q11-deletion [Bassett et al., 1998], while in other subjects, other genetic or non-genetic factors underlie the disorder. We do not know how often single gene defects or variants occur in a large population of subjects with a particular psychiatric phenotype: a large number of single gene disorders is still unknown, and so is their prevalence. These conditions are not necessarily very rare: $22 \mathrm{q} 11$-deletion and the $N F$ l-gene mutations are present in 1 in 4000 individuals. Moreover, single gene disorders are not always associated with a recognisable physical phenotype: in X-linked mental retardation (XLMR) at least 33 genes on the X chromosome are associated with moderate or mild mental retardation in males, without being associated with a particular physical phenotype [Chiurazzi et al., 2001]. It is possible that these so-called non-specific XLMR-genes are actually associated with specific behavioural phenotypes [Fisch, 2000].

To understand the possible effects of single genes on behaviour, it is important to understand the mechanisms that can explain the large variability in phenotype. History has shown that discovering such a mechanism in one gene can help to discover the gene-phenotype pathways in other genes in which a similar mechanism operates. When intergenerational amplification of a trinucleotide repeat was discovered as the underlying molecular mechanism in fragile-X syndrome, this helped elucidate the phenomenon of anticipation' in several neurological diseases [Petronis and Kennedy, 1995]. The different impact of a single gene on the phenotype can be explained by some of the mechanisms discussed below, though this overview is certainly not exhaustive.

\footnotetext{
'Anticipation means that a particular hereditary disease occurs a younger age and has a more severe course in offspring than in parents.
} 


\subsection{Phenotypical differences and genotypical heterogeneity}

One reason why the phenotype of a clinical disorder can differ in severity between subjects is the fact that the disorder may have different, though often related, genetic causes. In earlyonset Alzheimer's disease (EOAD), at least three different genes coding for enzymes in the biochemical pathway of amyloid $\beta$ peptide lead to a similar neuropsychiatric disorder, though the average age of onset of EOAD may differ depending on which of the genes is mutated [Cruts and Van Broeckhoven, 1998].

Another form of genetic heterogeneity is when different mutations of a disease gene coexist in the population, each of them with a different impact on the phenotype. In the autosomal recessive metabolic disorder phenylketonuria (PKU), a deficit of the enzyme phenylalanine hydroxylase results in elevated levels of phenylalanine and a varying degree of mental retardation when untreated. Different mutations in this gene result in varying degrees of elevated phenylalanine and mental retardation [Guttler and Guldberg, 2000]. In cystic fibrosis (CF), an autosomal recessive disorder with severe pulmonary and pancreatic involvement, at least 300 different mutations in the CFTR-gene have been described [Claustres et al., 2000]. These different mutations have a different impact on the phenotype: the typical severe CFphenotype is associated with a "severe" mutation on each of both alleles, while "milder" mutations on both alleles or a severe mutation on one allele combined with a normal allele, are associated with bilateral absence of the vas deferens in males, but not with CF.

\subsection{Dynamic repeats, anticipation, variable expression}

In the genome many forms of particular sequences of two, three or four base pairs occur. These di- tri- or tetranucleotide repeats are generally stable. The term "dynamic mutations" is used for these polynucleotide repeats that may become unstable and expand over generations [Richards R.I., 2001]. A CGG-trinucleotide repeat was first described in the Fragile X Mental Retardation gene (FMRI-gene) [Yu et al., 1991], and later found to be the cause of the fragileX phenotype [Verkerk et al., 1991]. Healthy subjects have less than 50 CGG repeats, and this number is stable from parents to offspring. Normal individuals can however also be carrier of a so-called premutation, with 50 to 200 CGG repeats. This number of CGG repeats becomes unstable when transmitted by a female. Once the expansion exceeds 200 CGG repeats, the full mutation is present. Translation of the FMRI-gene is impaired, resulting in absence of the FMR1-protein [Feng et al., 1995], expressed in the fragile-X phenotype. This mechanism may take several generations before the syndrome occurs. In fragile- $\mathrm{X}$ syndrome, the phenotype varies in a rather dichotomous way: males with the premutation do not have the fragile- $\mathrm{X}$ 
phenotype, while males with the mutation do. Dynamic mutations are the cause of at least seven disorder phenotypes (see Table 5) [OMIM, 2002; Richards R.I., 2001]. In some of these disorders, e.g. Friedreich's Ataxia and SCA, there is additional genetical and phenotypical heterogeneity. It is striking that all the dynamic mutations that have been described until now primarily affect the central nervous system. In trinucleotide repeat disorders, the severity of the phenotype may depend on the expansion length of the triplet repeat, and there are often threshold effects. In Huntington's disease the disease occurs above a threshold of 37 CAG repeats in the gene, and above that threshold longer repeats are associated with an earlier age of onset and a more malignant disease process [Brandt et al., 1996]. It has been suggested that also in multiplex families with major psychoses an expanding trinucleotide repeat might explain the finding that the disease process can get worse in consecutive generations (anticipation) [Petronis and Kennedy, 1995]. This hypothesis has not been confirmed sofar.

Table 5 Overview of central nervous system disorders associated with dynamic mutations.

\begin{tabular}{ll}
\hline Disorder & Mutation \\
\hline Fragile X syndrome & (CCG)n repeat expansion in promoter region of FMR \\
& gene; locus Xq27.3 \\
Myotonic Dystrophy & (CTG)n repeat expansion in untranslated region of \\
& DMPK gene; locus 19q13.3 \\
Huntington's chorea & (CAG)n repeat expansion in huntingtin gene; locus \\
& $4 \mathrm{pl6.3}$
\end{tabular}

Spinocerebellar ataxias type 1-17 (SCA1- At least 17 different genes. In at least 7 of these, the SCA17). mutation is a dynamic (CAG)n repeat expansion.

Friedreich's ataxia (FRDA) (GAA)n repeat expansion in frataxin gene; locus $9 \mathrm{q} 13$. Other gene (frataxin-2) at locus $9 \mathrm{p} 23$.

Dentatorubral pallidoluysian atrophy

(CAG)n repeat expansion in atrophin-1 gene; locus 12 p13.31

Spino-bulbar muscular atrophy (Kennedy (CAG)n repeat expansion in androgen receptor gene*; disease) locus Xq11-q12

The data are based on "Online Mendelian Inheritance in Man"-database [OMIM, 2002]. This overview does not include disorders that have only been found in a very small mumber of subjects. - Smaller than average repeat lengths at this site possibly result in a higher risk for prostate cancer [Irvine et al., 1995].

Another aspect of trinucleotide expansions is that we do not know much about the phenotype of expansions that exceed the normal range but which are not associated with the classical phenotype of that disorder. Some findings suggest that in some genes, intermediate repeat lengths could be associated with different phenotypes. The fragile- $\mathrm{X}$ premutation, an 
expansion of 55 to 200 CGG repeats near the FMRI-gene is not associated with the fragile-X phenotype. However, there is strong evidence that female carriers of the premutation are at risk for premature ovarian failure [Allingham-Hawkins et al., 1999], and there are suggestions that a number of them have a different cognitive development [Steyaert et al., 2002; Tassone et al., 2000c]. In the SCA8 gene, an expansion between 107 and 127 CTG-repeats is associated with Spinocerebellar Ataxia, while much longer repeats are associated with major psychosis in some individuals, and not with Spinocerebellar Ataxia [Vincent J.B. et al., 2000]. It is clear that the importance of trinucleotide repeats in neuropsychiatric disorders should be studied further.

\subsection{Sex effects}

An obvious reason for phenotypical variation is sex effect in X-linked disorders. Females can benefit from the compensatory effect of the homologue gene on their second X-chromosome. In some conditions, e.g. colour blindness, females will be clinically unaffected carriers of the gene. In other conditions, e.g. fragile-X, females manifest a broad phenotypical spectrum, ranging from complete normality to moderate mental retardation. However, more indirect sex effects also exist in the expression of genes associated with particular phenotypes. Genetic factors play an important role in the autism phenotype. Autism is strikingly more frequent in males than in females, but no data have been found in favour of the location of "autism" genes on the $\mathrm{X}$ chromosome. Therefore, it is assumed that pre-natal hormonal effects may play a role in the expression of the autism phenotype [Manning et al., 2001].

\subsection{Effects of other genes}

Other genes can affect the outcome in a single gene disorder. The effect of a defective gene can depend on the functioning of another gene. In a number of metabolic pathways, poor or absent expression of two genes may be necessary before any phenotypical effect is present. This is of course the case in autosomal recessive disorders, where the presence of one normal copy of the gene is sufficient for normal functioning, while absence of both alleles causes disease. In addition, more subtle mechanisms exist: in some monogenic mental retardation disorders, the mutated gene has such an impact on cognitive function that the level of mental retardation is independent of other factors. In other disorders, though the affected subjects have a marked cognitive impairment, the intelligence quotient correlates with the cognitive level of the subject's parents. This was demonstrated in VCFS (22q11-deletion) [Swillen et al., 1997]. It is assumed that in VCFS "background" genes still influence intelligence, while they have no effect in Fragile $\mathrm{X}$ syndrome. 
It becomes even more complicated when mutations in several different genes are necessary for the disease to occur, as suggested in the theory of polygenic inheritance of disorders. Few examples in molecular genetics support this. One example is that of Bardet-Biedl syndrome (BBS), an autosomal recessive disorder including pigmentary retinal dystrophy, polydactyly, obesity, developmental delay and renal defects. Two different genes for BBS have been found, BBS2 and BBS6 and it was expected that subjects with two mutated BBS2 or BBS6 genes would manifest the BBS phenotype [Katsanis et al., 2001]. However, in the studied pedigrees subjects were found with two mutated BBS2 genes and a normal phenotype, while an additional mutation in one of the BBS6 genes is necessary to manifest the BBS phenotype.

\subsection{Distant effects of gene defects}

Gene defects can have molecular consequences that extend far beyond the obvious pathway of gene, gene product and the metabolic function of the gene product. Firstly, through feedback mechanisms, poor or absent expression of a gene may increase translation to messenger RNA (mRNA). Eventually this specific mRNA will accumulate. There is evidence that in Myotonic Dystrophy, the myopathy is caused by the mutant mRNA, while cardiac conduction defects and cataracts are caused by a deficiency in the DMPK protein, the gene product of one of the two known genes responsible for Myotonic Dystrophy [Tapscott and Thornton, 2001]. In carriers of the fragile-X premutation the mRNA levels of the FMRI-gene are much higher than in normal controls [Tassone et al., 2000b], and it is thougth that this may play a role in the phenotypical anomalies found in some fragile- $\mathrm{X}$ premutation carriers (see above).

Secondly, the absence of a particular gene product may have positive or negative feed back effect on the translation of other genes. In mice without FMRI-gene (knock-out fragile-X mice), the expression of at least 200 genes has changed quantitatively [Gantois and Kooy, 2002; Gantois et al., 2002].

Thirdly, in neurotransmitter metabolism changes in a coding gene may alter the efficiency of an enzyme, resulting in other metabolic equilibriums and consequently different concentration of the neurotransmitter. In Brunner's syndrome [Brunner et al., 1993] the MAO-A deficiency has complex effects on the function of several neurotransmitters and it is not clear which of these changes affect behaviour [Brunner, 1996].

\subsection{Chance}

Randomness is a fundamental characteristic of probabilistic events and consequently these are difficult to describe. However, stochastic effects (the cumulative effect of probabilistic events) play a role in the development of a complex organism. This is one of the reasons why 
monozygotic twins (MZT) are not completely similar and may have different anatomical defects although they share the genetic susceptibility for this defect. Individuals with trisomy 21 or 22q11-deletion often have atrioventricular septum defects (AVSD). However, MZT pairs with these genetic syndromes may be discordant for cardiac defects [Vincent M.C. et al., 1999]. Even in one individual, a gene defect may affect one side of the body and not the other, as seen in the Bardet-Biedl syndrome (see above), where the polydactyly may only affect one hand and not the other. This phenomenon of asymmetry due to probabilistic events can also be observed in normal development: e.g. selective breeding shows that the average length of the white leg markings in horses is hereditary, but the differences of these white markings between left and right legs are probabilistic and not determined by genes [Woolf, 1995]. To demonstrate the stochastic basis of closure of the atrioventricular septum, a computer model simulating the growth of the septum between the two endocardial cushions during embryonic development was made [Korenberg and Kurnit, 1995]. The septum closes through cell division, migration and adhesion. The computer model allowed for skewing of (genetically determined) parameters for cell division, migration and adhesion between cells, as seems to be the case in trisomy 21 . Consecutive runs of the computer program with an identical skewing resulted in non-closure of the atrioventricular septum in a number of runs, but not in others. Thus, changing these genetically determined parameters resulted in an increased chance to develop an AVSD, but did not predict normal or abnormal development in a dichotomous way. This is exactly what happens in trisomy 21 , where some children with Down syndrome are born with AVSD and others with a normal heart, even in monozygotic twins. This shows that stochastic effects can yield significant phenotypic variability among individuals with identical genotypes and environments.

\section{Discussion}

Quantitative behaviour genetics investigate to what extent a particular behavioural trait or disorder can be genetically determined and which mechanisms may be involved in its heredity. Molecular behaviour genetics investigate which genes can be involved in behaviour and psychiatric disorders and by which mechanisms these genes operate. In the past few decades, quantitative and molecular behaviour genetics have yielded important insights in developmental psychiatry and valuable new techniques in both fields. Though psychiatric disorders are often strongly genetically determined, they cannot be seen as one-gene-onedisorder diseases [Plomin et al., 1994]. Their heredity generally does not follow classic 
Mendelian rules, and more complex forms of heredity have been suggested. Quantitative genetics has provided strong arguments in favour of polygenic causality. According to this model, a large number of genes, or quantitative trait loci, in interaction with environment factors, have an additive effect contributing to the psychiatric phenotype. These studies necessitate large samples of patients and families, which are inevitably genetically heterogeneous. The examples given in this review compel us to assume that a large sample with a particular phenotype, e.g. schizophrenia, consists of at least a subgroup in which the additive effect of a particular set of genes is responsible for the phenotype, and a subgroup in which a number of isolated genes, or very few genes cause the phenotype. One argument in favour of the existence of this mono- or paucigenic group is that some single gene disorders and isolated chromosomal defects are associated with a psychiatric phenotype. A second argument is that the few molecular studies on polygenic mechanisms have showed so far that even when the additive effect of a large number of genes is analysed, this only explains a modest part of the variance, even in disorders with a high hereditability [Comings et al., 2000]. To complicate matters, the polygenic group may in its turn consist of several subgroups in each of which a different set of genes contributes to the phenotype. However, there are no simple clinical means to separate the polygenic and the mono- or paucigenic groups, and we do not know their relative importance and contribution.

Differentiation of the phenotype may be a rewarding technique to obtain different and genetically more homogeneous subgroups in these samples, which in their turn would facilitate the search for the involved genes. By differentiating the phenotype "early onset Alzheimer's Disease", this method led to the discovery of genes that play a role in Alzheimer's disease. However, we have only dim cues on how to achieve such a redefinition of phenotypes. Ideally, it should be based on genetic characteristics, and that is exactly what we are looking for and do not have yet. Eventually, research based on other phenotypic categories might lead to the conclusion that the relation between genes and phenotype is often too complex and indirect, or should be studied in a different way.

Considering these methodological difficulties in the study of gene-behaviour pathways using a backward approach starting from the phenotype side, it is prudent also to study what we can learn when starting from the side of molecular biology, or forward approach. Disorders involving only one or very few genes can have a phenotypic expression that is much more variable than initially expected, as we have illustrated above. In the past 10 years, in-depth study of the moleculat biology of some of these disorders has revealed formerly unknown mechanisms, which operate in more genes and disorders. Simultaneous effect of other genes, 
distant effect of gene defects, dynamic mutations, probalistic mechanisms, and yet undiscovered mechanisms explain the broad phenotypic variance of single gene disorders and their seemingly non-Mendelian patterns of inheritance. They illustrate how complex the genebehaviour pathway already is when the genetic causes are comparatively simple, and give us a faint idea of the complexity of the problem when a large number of genes are involved. The rule of parsimony in research suggests studying first how single gene may affect neuropsychiatric phenotypes. We do not know the prevalence of single gene disorders in a population with a particular psychiatric phenotype, but can learn a lot from the study of phenotypes associated with single gene disorders. First, it gives insight in the pathway between that particular gene and behaviour. Secondly, through showing which behaviour is influenced by a particular gene, it may help to redefine the phenotype of neuropsychiatric disorders, and thus open new alleys in gene-behaviour research of these disorders. Thirdly, it may lead to previously unknown mechanisms in molecular biology. One way to do this is to study individual patients or families in whom a neuropsychiatric disorder is associated with a demonstrable gene defect. This is the case in chromosomal rearrangements, microdeletions, or microduplications. In chromosomal rearrangements, the genes disrupted at the breakpoints can be identified. In microdeletions, the effect of deleted genes can be analysed, as is the case in the association of schizophrenia with 22 -deletion. In microduplications, we can study the effect of redundant genes, as in the recently discovered association between anxiety disorders and a microduplication on chromosome 15 [Gratacos et al., 2001]. Fourthly, better knowledge of the building blocks may improve our understanding of how multiple genes operate together. Finally, focussing on single genes in an array of QTL-genes underlying a neuropsychiatric disorder, may open new alleys to treatment, as was illustrated by the finding that children with $\mathrm{ADHD}$-a polygenic disorder- have different therapeutic responses to methylphenidate depending on which alleles of the dopamine receptor D2 genes they have [Winsberg and Comings, 1999].

Clarifying pathways and hopefully phenotypes using this molecular genetic method will probably lead to new approaches in quantitative genetics. This may allow new insights into the genetics of polygenic neuropsychiatric phenotypes, and will certainly bring both fields of research even closer to each other. 

Chapter 4 Fragile $X$ syndrome 


\section{Introduction}

Fragile-X syndrome (FXS) is the most frequent form of hereditary MR in males. Its prevalence in the general population is estimated between $1 / 1000$ and 1/4000 males [Turner et al., 1996]. More recent prevalence estimations are lower than the earlier ones. The syndrome has an X-linked semi-dominant heredity pattern. It can affect several subjects in one or more branches of a family in which MR had not occurred earlier. The syndrome and its particular pattern of heredity are caused by a dynamic mutation: an unstable expansion of a trinucleotide (CGG)n repeat sequence in the promoter region of the FMRl-gene, located at $\mathrm{Xq} 27.3$. This mechanism was discovered in 1991 [Verkerk et al., 1991]. Before the mutation could be detected by molecular techniques, the diagnosis of FXS was made by demonstrating the fragile site on the X-chromosome in a lymphocyte culture using an adapted medium [Sutherland, 1977].

There are two levels of CGG-repeat amplification (see this chapter, section 5). In the premutation, with expansions of less than 200 repeats, the gene is still expressed and the individual does not have the classical fragile-X phenotype. These males and females transmit the fragile $\mathrm{X}$ gene to the next generation. In the full mutation, the expansion exceeds 200 repeats, the FMRI-gene is not expressed anymore and the classical fragile-X phenotype occurs. Males with the full mutation are usually significantly affected. In females with the full mutation, the degree of impairment varies widely.

\section{Clinical phenotype}

The classical phenotype of FXS is found in affected adult males. The prominent clinical features are moderate mental retardation, associated with macro-orchidism and typical craniofacial features: long and narrow face with prominent forehead and lower jaw, and large ears [Fryns J. P. et al., 1984a]. Other characteristics include relative macrocephaly, joint hyperlaxity, muscle hypotonia, arched palate, mid-face hypoplasia with sunken eyes, and seemingly benign mitral valve prolapse [Laxova, 1994]. Several of these signs have been attributed to a connective tissue disorder [Opitz et al., 1984; Sreeram et al., 1989].

\subsection{Mental retardation}

The most salient aspect of the behavioural phenotype is MR. Adult males have an average mental level in the moderately mentally retarded range. Some have a severe MR and exceptionally a mild MR. Pre-pubertal boys, and in particular pre-schoolers, have a better 
average mental age than adolescents and adults [Borghgraef et al., 1987]. Longitudinal analysis demonstrated that individual subjects show a decline in cognitive level and adaptive behaviour skills [Borghgraef et al., 1995; Fisch et al., 1996; Hagerman R.J. et al., 1989]. The decline occurs in all tested cognitive areas: quantitative skills, verbal reasoning. visual/abstract abilities and short-term memory [Fisch et al., 1996]. It is not clear whether this decline is limited to the period of development from childhood to adolescence, or whether it continues far into adulthood. The latter was suggested in a cross-sectional study [Maes et al., 1994] but not confirmed by longitudinal studies.

\subsection{Social contact}

Initial shyness and gaze aversion are often present. In most fragile $\mathrm{X}$ males, social skills are in accordance with the cognitive level. Some subjects with FXS may have autistic features, in particular when their mental handicap is severe. Once it was thought that FXS could be the cause of many cases of autism [Brown et al., 1982; Hagerman R.J. et al., 1986b]. The latter view was refuted by showing that autism does not occur more frequently in FXS than in other subjects with the same level of MR [Fisch, 1992].

\subsection{Hyperactivity and attention deficits}

Attention-deficit and Hyperactivity Disorder (ADHD) symptoms are a part of the behavioural phenotype in most boys with FXS [Borghgraef et al., 1987; Fryns J. P. et al., 1984a; Hagerman R. et al., 1985]. These symptoms tend to decline with age, but many adult males with FXS are still quite active. Treatment with stimulant medication has proven to be successful [Hagerman R.J. et al., 1988] in a number of cases. Observations of subjects with FXS suggest that the symptoms of $\mathrm{ADHD}$ may at least partly be due to sensory hyperreactivity and lack of stimulus inhibition [Boccia and Roberts, 2000; Miller et al., 1999]. This was also observed in knockout mouse models of FXS [Chen and Toth, 2001].

\subsection{Language}

Articulation is poor, and language is repetitive and characterized by short and fast utterances [Borghgraef et al., 1987]. Cluttering is often present [Hanson et al., 1986]. Language acquisition is slow and in adult FXS males it tends to plateau at a level of about 4 years [Fisch et al., 1999b]. Their vocabulary level is similar or sometimes better than that of subjects of the same mental level [Maes et al., 1994]. 


\subsection{Other characteristics:}

Hand biting, hand flapping, and fast rocking are often observed [Bardoni et al., 2000]. These symptoms occur in particular when subjects are excited or over-stimulated, and are probably a sign of their hyperreactivity.

A benign form of epilepsy is present in approximately one quarter of young males with FXS [Musumeci et al., 1991; Sabaratnam et al., 2001]. It disappears after adolescence in half of the affected subjects.

\section{Females with FXS}

In females with the full mutation, physical features resemble those observed in males with FXS [Hull and Hagerman, 1993; Riddle et al., 1998]: long face, prominent forehead and jaw, joint hyperlaxity. They present a higher variability of the physical phenotype, due to individual differences in the activation ratio of the $\mathrm{X}$-chromosome carrying the mutated FMRI-gene.

Early studies already found cognitive impairment in a number of heterozygous carriers of the fragile X chromosome [Fryns J. P. et al., 1984b; Fryns J. P., 1986; Nielsen et al., 1981; Uchida and Joyce, 1982]. Studies that are more recent show that at least 50 to $60 \%$ of women with the full mutation are cognitively impaired. In these subjects, cognitive impairment correlates with the activation ratio of the fragile X chromosome [Abrams et al., 1994; Willemsen et al., 2000], rather than with the length of the amplification. A higher activation ratio of the fragile $\mathrm{X}$ chromosome is associated with lower intelligence. Like in males with FXS, a cognitive decline in full mutation fragile $\mathrm{X}$ females has been observed [Fisch et al., 1999a].

Before the fragile mutation was found and female pre-mutation carriers could be delineated from full mutation carriers, it was demonstrated that heterozygotes carrying the fragile $\mathrm{X}$ chromosome and with an IQ in the normal range (full scale IQ higher than 85 ) have more specific cognitive problems than expected (see also 0) [Brainard et al., 1991; Kemper et al., 1986; Miezejeski et al., 1986; Steyaert et al., 1992; Wolff et al., 1988]. A significant increase of low academic performance was found in these heterozygote carriers with normal intelligence [Steyaert et al., 1992; Wolff et al., 1988]. This finding became clearer after 1991, when DNA analysis of the fragile $\mathrm{X}$ mental retardation gene (FMRI) became possible. The presence of cognitive deficits in full mutation carriers was confirmed by several studies [Franke et al., 1998; Thompson et al., 1994]. The specific neurocognitive problems found beside mental retardation are: impairment of executive functions [Bennetto et al., 2001; 
Franke et al., 1999; Thompson et al., 1994], attention deficits [Franke et al., 1999], and impairment of visuo-spatial functions [Bennetto et al., 2001; Cornish et al., 1999].

Several studies describe excessive shyness, avoidant disorder, social phobia or selective mutism in girls and women with the full mutation [Franke et al., 1998; Freund et al., 1993; Hagerman R.J. et al., 1992; Hagerman R.J. et al., 1999]. Other behavioural characteristics include schizotypal personality characteristics [Sobesky et al., 1994a] and a so-called fakinggood profile on a personality questionnaire [Sobesky et al., 1994b; Steyaert et al., 1994]. Women with the full mutation have a significantly higher lifetime prevalence of major depressive disorder than mothers of similarly handicapped children without FXS [Thompson et al., 1996].

\section{Premutation carriers}

The case of premutation is more equivocal. Initially, the premutation was thought not to affect their cognitive functioning [Mazzocco and Holden, 1996; Reiss et al., 1993]. Recent research in children carrying the premutation led to the same conclusion [Myers et al., 2001]. This view was challenged gradually as minor anthropomorphic anomalies were described in female and male premutation carriers [Hull and Hagerman, 1993; Loesch et al., 1994; Riddle et al., 1998].

Female premutation carriers have a significant increase of premature ovarian failure (POF) [Allingham-Hawkins et al., 1999], while this is not a characteristic of full mutation carriers [Murray et al., 2000]. The neurocognitive findings in female premutation carriers are equivocal: while some researchers [Franke et al., 1999; Mazzocco and Holden, 1996] did not find neurocognitive deficits in female premutation carriers, others found moderate cognitive deficits in two out of six premutation carriers [Tassone et al., 2000c]. Some authors have found mild emotional impairment in females with a "long premutation" between 100 and 200 repeats, compared with females with shorter premutations [Johnston et al., 2001]. The authors interpreted the findings as a mild manifestation of the classical phenotype in females with the full mutation. Others found no cognitive impairment in female premutation carriers [Franke et al., 1999]. These studies are difficult to compare, as they used different approaches in assessing the group of subjects. Some delineated different subgroups, while others clustered all premutation carriers. In some studies, only a decline in IQ was considered as relevant, while others took specific neurocognitive differences into account. A study by our research group (see Chapter 7), using the latter approach, suggests that some female carriers of the premutation have distinct cognitive characteristics, which are qualitatively different from the 
findings in female carriers of the full mutation [Steyaert et al., 2002]. Like the finding of POF in premutation carriers and not in full mutation carriers, the latter neurocognitive finding supports the hypothesis that there are two different phenotypes.

There is less information regarding male premutation carriers, or transmitting males. In some cases, learning disabilities in males in the upper premutation size range have been observed [Hagerman R.J. et al., 1996]. It is not clear whether the findings of these case studies are applicable to the whole group of transmitting males.

\section{Fragile $X$ syndrome: molecular biology}

\subsection{The FMRI-gene}

The fragile X Mental Retardation gene $(F M R I)$ is located on Xq27.3. It spans about $40 \mathrm{~kb}$ and is composed of 17 exons, encoding a messenger RNA (mRNA) of $3.9 \mathrm{~kb}$ [Bardoni et al., 2000]. There is a cytosine-guanine-guanine (CGG) repeat of varying length at the 5' untranslated region of the gene, in the first exon of the FMRI-gene (Figure 1). It is part of a CpG island that extends upstream of the transcription initiation site (promoter region) and plays an important role in FMRI-expression. Most individuals carry 6 to 54 CGG repeats at this site [Fu et al., 1991]. Approximately 1 in 510 X chromosomes carries a fragile X premutation: a FMRI-gene with 55 to 200 repeats [Reiss et al., 1994a; Rousseau et al., 1995]. Transcription of the gene carrying this premutation is normal, though translation may be impaired in the upper premutation repeat size range [Feng et al., 1995]. There is an overlap or grey zone between the CGG repeat lengths found in the general population (6-54) and the repeat lengths found in premutation carriers in FXS families (50-200) [Fisch et al., 1994].

Methylation of the CpG island occurs when the CGG repeat expansion exceeds 200 repeats and results in silencing of the FMRI-gene [Hansen et al., 1992]. Even when hypermethylation is not present, there is diminished translation of mRNA transcript containing a repeat in the upper premutation range, close to 200 repeats [Feng et al., 1995]. When the expansion exceeds 200 repeats, $40 \mathrm{~S}$ ribosomal subunits stall and translation is strongly diminished.

While alleles with a normal repeat length are stable in the general population, alleles with a premutation size repeat are unstable and tend to expand from one generation to the next through maternal inheritance, but not through paternal inheritance [Smits et al., 1992]. Present knowledge indicates that the expansion to longer repeat lengths is a prezygotic event, which probably occurs in oogenesis [Moutou et al., 1997], whereas a selection for cells expressing FMRP, or for a shorter CGG repeat, occurs in fetal spermatogonia [Malter et al., 1997]. 
Figure 1 The FMRI-gene

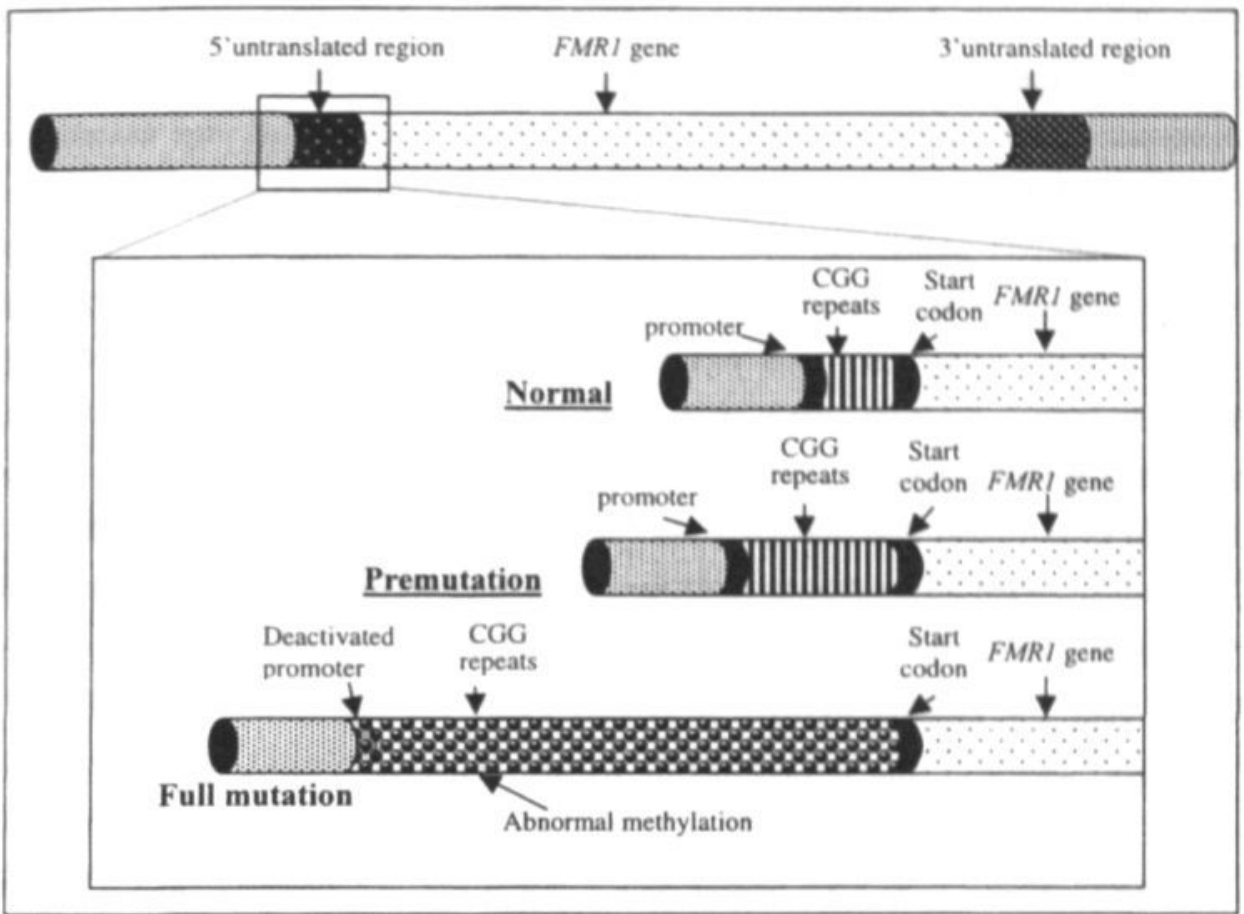

The trimucleotide expansion responsible for fragile $X$ syndrome lies in an unexpressed part of the FMRI gene. The gene itself (top) divides into 17 exons spread over 38 kilobases. Its first and last exons include regions transcribed into messenger RNA that are not represented in the final translated protein. In turn, the 5 , untranslated region includes sequences of CGG repeats. Normally, the tract is polymorphic, ranging from 7 to 50 repeats. In a premutation, the number is 55 to 200 . In a full mutation, the number is more than 200 . When the repeat number exceeds 230, the entire region is hypermethylated (insert), receiving a methyl group at the $C$ in each CG dinucleotide along both strands of the DNA double helix. The gene 's promoter is deactivated, and the gene becomes silent. Between 200 and 230 repeats, hypermethylation may be incomplete. Adapted from [Warren, 1997].

The CGG repeat length may vary in somatic cells of one individual. Mosaicism of mutation and premutation in one tissue occurs in as much as $41 \%$ of FXS males [Nolin et al., 1994] and different mutation lengths in one tissue are frequent. The latter causes a smearing phenomenon on Southern blot analysis of DNA with the FXS mutation. The phenomenon also occurs in male premutation carriers, who display both contractions and expansion of the repeat within the premutation range, in both sperm and somatic tissue [Nolin et al., 1999]. The repeat length may vary within one tissue, but there is only limited variation in the proportion of different repeat lengths in different tissues [Reyniers et al., 1999; Tassone et al., 1999a]. Relative homogeneity of the distribution of repeat lengths in different tissues is in accordance with the theory that expansion of the repeat is a very early phenomenon in development. Methylation mosaicism may also occur in FXS: in different cells the repeat may 
be methylated, while the same repeat length remains unmethylated in other cells [Genc et al., 2000; Steyaert et al., 1996; Tassone et al., 2000a; Wohrle et al., 1992]. The phenomenon occurs in subjects with the full mutation, and has been described in subjects with a premutation [Tassone et al., 1999b]. These two kinds of mosaicism may cause some differences in repeat lengths and methylation status between different tissues. Assessing repeat length and methylation status in brain tissue by measuring these parameters in peripheral lymphocytes, as is mostly done, may thus seem imprecise. With some exceptions [Dobkin et al., 1996], most cases reported, and in particular post mortem case studies including brain tissue, have shown strong similarity of the mutation size and methylation status between tissues [Reyniers et al., 1999; Tassone et al., 1999a]. The technique has been accepted to be reliable. This is supported by clinical research, e.g. the finding of a significant correlation between methylation status in peripheral lymphocytes and the level of cognitive impairment [Hagerman R.J. et al., 1994; Steyaert et al., 1996].

\subsection{The gene product: FMRP}

The FMRI-gene transcript is subjected to alternative splicing. Five or six isoforms are produced and are collectively termed Fragile X Mental Retardation Protein (FMRP) [Bardoni et al., 2000]. FMRP is an RNA binding protein that is part of a ribonucleoprotein particle associated with actively translating polyribosomes, and which can shuttle between nucleus and cytoplasm. Two highly homologous human proteins, FXR1P and FXR2P, share the same domain structure as FMRP, and probably have similar functions. The properties of FMRP suggested that it is involved in nuclear export, cytoplasmic transport, and/or translational control of target mRNAs. In particular, it may play a role in regulation of protein synthesis at postsynaptic sites of dendrites, and in maturation of dendritic spines [Bardoni et al., 2001; Braun and Segal, 2000]. Efforts are underway to identify the putative specific mRNA targets of FMRP, and to study the effect of the absence of FMRP on the corresponding proteins. In mice without FMRl-gene (knock-out fragile-X mice), the level of expression of at least 200 genes has changed [Gantois et al., 2002]. Other approaches have led to the identification of proteins that interact with FMRP. Some of them discriminate between FMRP and the homologous FXR 1/2P proteins, and may thus be important for defining unique functions of FMRP that are deficient in Fragile X patients [Bardoni et al., 2001]. There is evidence that FMRP causes dose-dependent translation inhibition of some proteins expressed in the brain [Li et al., 2001]. 
The gene is widely expressed, though not everywhere, and in different quantities. FMRImRNA and FMRP are particularly abundant in neurons, in particular in the hippocampus and the cerebellum [Abitbol et al., 1993; Bardoni et al., 2000]. High concentrations of FMRP are also present in epithelial tissues and in spermatogonia [Bachner et al., 1993]. In the mouse embryo, FMRI-expression is high during early development and decreases progressively afterwards. In human embryos, there is high expression of the FMRI-gene in proliferating. migrating and differentiated neurons. This suggests that FMRP plays an important role in early brain development [Agulhon et al., 1999].

The FXS is associated with lack of expression of the FMRI-gene and absence of FMRP [Ashley et al., 1993; de Vries et al., 1998]. In individuals carrying the premutation expression of FMRP is mainly normal. In subjects with 100 to 200 CGG repeats, normal levels of FMRP are associated with increased levels of FMRI-mRNA [Tassone et al., 2000b]. The increased levels of FMRI-mRNA are caused by increased transcription of the FMRI-gene. This is probably a mechanism that compensates for diminished translational efficiency of varying degree throughout the whole or a part of the premutation repeat size range. The increase of FMRI-mRNA in the cell may have an effect on the expression of proteins other than FMRP. This phenomenon has been observed in Myotonic Dystrophy, where intracellular accumulation of mRNA transcripted from the mutated gene causes some symptoms of the disease [Tapscott and Thornton, 2001].

\section{Patterns of heredity}

Once the full mutation is present in a family, the pattern of heredity is typically that of an Xlinked (semi-)dominant disorder (Figure 2). All males who carry the full mutation are affected, while females are variably affected. Random but individually different inactivation of fragile $\mathrm{X}$ chromosome in brain tissue is probably the cause of the variation of severity in female carriers of the full mutation. 
Figure 2 Four generations in a fragile $\mathrm{X}$ pedigree

Female mutation carrier

Male mutation carrier
- Female premutation carrier

- Male premutation carrier
Normal female

Normal male

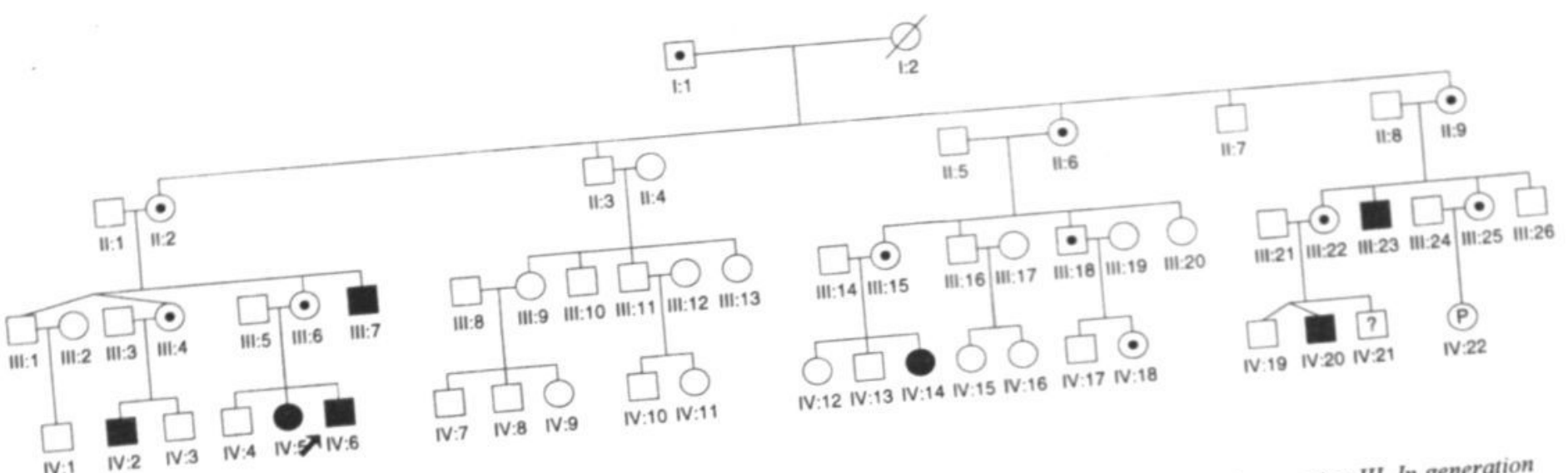

IV:

Subject IV 6 is the index patient. Note the absence of affected subect
$I V$, all branches with a carrier parent include affected patients. 
It is not clear what events affect transition from the normal size allele to the premutation size allele. When the premutation is present in a family, male and female carriers may transmit it to their children. When male premutation carriers, or normal transmitting males, transmit the premutation to their daughters, as a rule there is no expansion of the mutation. Reduction of the number of repeats occurs occasionally [Fisch et al., 1995]. When female premutation carriers transmit it to their daughters or sons, expansion of the premutation may occur, and result in a longer premutation or in a full mutation. Female full mutation carriers have a normal fertility and offspring who inherit their fragile $\mathrm{X}$ chromosome will similarly have a full mutation. In males with the full mutation, sperm may contain a variety of expansion lengths, including contractions to shorter alleles [Nolin et al., 1999]. This phenomenon is probably caused by embryonic selection for cells with a short repeat length amongst the spermatogonia (see above).

\section{Brain imaging}

Subjects with FXS have relative macrocephaly from early childhood [Fryns J.P., 1989]. Underlying this, there are anomalies of total brain volume, and of different brain structures. Magnetic resonance imaging (MRI) and quantitative morphometry showed that subjects with an FMRI-mutation have increased total brain volume, and relatively increased volume of the caudate nucleus and, in males, the lateral ventricle [Reiss et al., 1995]. Both caudate and lateral ventricular volumes are correlated with IQ. Caudate volume is also correlated with the methylation status of the FMR-I gene. Cerebellar vermis is decreased in males and females with FXS and fourth ventricle volume is increased [Reiss et al., 1991a; Reiss et al., 1991b]. Hippocampal volume is larger [Kates et al., 1997; Reiss et al., 1994b]

A recent study in female premutation carriers demonstrated anatomical and metabolic changes in this group [Murphy D.G. et al., 1999]. Remarkably, the anatomical changes were not or only partially similar to those found in full mutation subjects. Some findings where even the opposite of what was found in subjects with a full mutation. Compared with controls, fragile $\mathrm{X}$ premutation carriers had a significant decrease in volume of whole brain, and caudate and thalamic nuclei bilaterally, and increase in volume of hippocampus, peripheral CSF bilaterally, and third ventricle. These findings add to the hypothesis of a separate phenotype in the fragile $\mathrm{X}$ premutation.

To our knowledge, functional brain imaging has not been performed in males with FXS. In females with the full mutation, functional MRI was used to examine visuo-spatial working 
memory [Kwon et al., 2001]. This study showed that subjects with fragile $\mathrm{X}$ syndrome are unable to modulate activation in the prefrontal and parietal cortex in response to an increasing working memory load, and these deficits are related to a lower level of FMRP expression in FXS. This finding is in accordance with purely neurocognitive research that shows that executive functions and visuo-spatial abilities are impaired in females with FXS (see above).

\section{Causes of variation in the phenotype}

Though there are striking similarities in the behaviour and cognitive level of many individuals with FXS, there is also a remarkable variation in the phenotype. Obvious differences are caused by sex difference: as a rule female carriers of the full mutation are less affected than male carriers, as they can benefit from activation of the normal FMRI-gene in a number of cells. It is equivocal whether genetic background plays a role in the severity of the mental impairment. One study suggested that in female full mutation carriers, the cognitive impairment correlates with the activation ratio of the fragile $\mathrm{X}$ chromosome, but not with the cognitive level of their non-carrier sisters [de Vries et al., 1996]. A more recent study suggests that background genes do play a modest but significant role in the cognitive development females with the full mutation, but have no significant effect in full mutation males [DyerFriedman et al., 2002].

Methylation status of the $\mathrm{CpG}$ island in the FMRI-promoter region has a strong effect on the level of cognitive impairment. Males with similar repeat lengths, but with a different methylation status, have strongly dissimilar IQ's, dependent on their methylation status [Steyaert et al., 1996].

Environmental factors also influence cognitive outcome to a small extend [Dyer-Friedman et al., 2002]. 


\section{Chapter 5 The behavioural phenotype in Myotonic Dystrophy}

Steyaert J. (2002)

In: Gene Fisch (ed.) Genetics and Genomics of Neurobehavioral Disorders. Humana Press, NY. In press. 


\section{Introduction}

Myotonic dystrophy (Dystrophia Myotonica, DM) is a heritable, slowly progressive muscle disorder with involvement of multiple organ systems. Not only the muscles are affected, but also the eye, heart, gastro-intestinal tract, skin, immune system, endocrine system, skull, kidneys [Thornton, 1999] and brain [Ashizawa, 1998]. Moreover, these systems may be affected differentially; the age of onset is variable, as well as the course of the disease. The severity of the disease tends to increase from one generation to the next, while the age of onset decreases. This phenomenon is called anticipation. The underlying genetic mechanism of myotonic dystrophy is that of dynamic mutations, which it shares with fragile-X syndrome, Huntington's disease and almost 20 other diseases affecting the central nervous system. The molecular mechanisms of myotonic dystrophy have been partly elucidated in the last 10 years. All these characteristics make myotonic dystrophy "perhaps the most variable of human disorders" [Harper, 2001].

Myotonic dystrophy affects the brain, and patients with the disorder have a wide range of behavioural symptoms. Perhaps the most common symptom is daytime sleepiness. Children with myotonic dystrophy have mild to moderate developmental delays, or may have more specific behaviour problems. In this chapter, after a review of the clinical and genetic features of the disease, I will focus on the effects of myotonic dystrophy in brain and what is known about the consequent cognitive and behavioural problems.

\section{History}

Myotonic disorders are characterized by myotonia, the stiffness of a muscle after contraction. The first description of this rare clinical sign was made in 1876 by Dr. Julius Thomsen, a German neurologist who was affected himself. He described a benign hereditary myotonia beginning in early childhood in his family: Thomsen's disease or myotonia congenita. In the following years, it became clear that a variant of this clinical entity existed. The new variant started later in life, and was accompanied by progressive muscle weakness and muscle wasting. In 1909, Steinert, another German neurologist, delineated this new hereditary myotonia as a separate entity, which was later named after him. Steinert was the first to describe clinical signs in systems other than the muscles. Later, the disease was called dystrophia myotonica, referring to the combination of muscle wasting and myotonia.

Since then, considerable progress has been made on the clinical and genetic aspects of this disease. Research in myotonic dystrophy can be arbitrarily divided in three phases: an early 
phase, approximately from 1909 to the 1970 s, during which most of the symptoms were described by experienced clinicians who reported case studies or small groups of patients. The second phase of research in myotonic dystrophy is from the 1970s to 1992, when the discovery of the gene and the mechanism of the mutation were published [Aslanidis et al., 1992; Buxton et al., 1992; Harley et al., 1992a]. This phase is characterized by systematic clinical studies on one hand and a hunt for the myotonic dystrophy gene on the other. In this "middle phase", it became clear how variable the phenotype of myotonic dystrophy could be. Unfortunately, knowledge of the different subtypes was not universally shared. Consequently, many high quality studies contain heterogeneous groups of subjects and are difficult to compare with each other. The present phase of research on myotonic dystrophy began with the discovery of the mutation, after which the diagnosis could easily be made through a DNA test. This phase is characterized by fundamental research on the pathway between the mutation and phenotype, clinical studies on different aspects of the disease, and in specific forms of myotonic dystrophy. Several clinical studies have looked for correlations between clinical characteristics and length of the mutation (see section 4.2 , this chapter). One of the consequences of the easy and definite diagnosis through DNA testing is that subjects with a mild form of the disease, or at a very early stage of the disease, can be included. For instance, this has led to the finding that in a particular form of the disease, namely the juvenile onset myotonic dystrophy, behavioural symptoms can precede neuromuscular symptoms [de DieSmulders C., 2000; Steyaert et al., 2000].

\section{Clinical aspects}

\subsection{Prevalence}

Myotonic dystrophy is the most common of myotonic diseases. Its prevalence is estimated at around one in 8000 persons [Wieringa, 1994]. However, this may be an underestimation as it is based on the prevalence of the congenital and classical or adult type of the disease, and probably does not include patients who were not diagnosed because they had not yet manifested significant symptoms.

\subsection{The classical type of myotonic dystrophy}

The classical form of myotonic dystrophy begins in late adolescence or adulthood, generally in the third or fourth decade of life (see Table 6 for overview of symptoms). Retrospectively, it is often difficult to pinpoint the time when symptoms first occur. Patients may have had vague cognitive or gastro-intestinal complaints well before any neuromuscular sign appeared. 
Clinicians who are not very familiar with the disease may not interpret this as possible early symptoms of myotonic dystrophy. Before the first complaints of muscle weakness, the disease often begins with myotonia: muscles that relax only gradually after an effort. This phenomenon can impair smooth and precise manual activities. It becomes more severe in cold weather, and is most pronounced after forceful movements have been made, hand movements in particular. Most patients experience this only as a minor discomfort and rarely seek medical attention for the problem. Tongue fasciculations, fine and rapid uncontrolled movements of the tongue muscles, are another sign of myotonia.

Muscle weakness generally begins in the face, jaw, neck and forearms. Patients typically exhibit little facial expression, and have progressive ptosis of the eyelids, wasting of the temporal and jaw muscles, articulation and swallowing problems. Gradually, the muscle wasting and weakness worsens and patients may eventually become wheel chair bound with severe respiratory problems, partly due to weakness of the diaphragm and other respiratory muscles.

One of the most common complaints in adult myotonic dystrophy patients is somnolence during the day. Patients can fall asleep anywhere and at any time. Besides these prominent and progressive muscle problems and typical daytime sleepiness, other organ systems are involved to a varying degree.

A frequent non-muscular symptom of myotonic dystrophy is progressive cataract: the eye lens becomes gradually more opaque and sight is impaired. In some patients with myotonic dystrophy, cataract may be the only symptom. These will often occur in members of the older generations in families with myotonic dystrophy, while members of younger generations are more severely affected, evincing symptoms in several organ systems. This phenomenon puzzled early researchers on myotonic dystrophy (Fleisher, (1918) cited by Harper [2001]) and could be explained once the molecular mechanism of a dynamic mutation (see this chapter, section 4.1) was found to cause myotonic dystrophy.

Myotonic dystrophy not only affects skeletal muscles, but smooth muscle tissue also. Smooth muscles are responsible for the motility of the gastro-intestinal tract from the pharynx and the oesophagus to the anal sphincter. Smooth muscles control the functions of the urinary bladder, as well as the working of the iris and the lens in the eye. In myotonic dystrophy, the smooth muscles of the gastro-intestinal tract are generally the most affected and this leads to a wide range of complaints and symptoms. Involvement of the pharynx and oesophagus can lead to swallowing problems and frequent tracheal aspiration. Problems of the colon range from vague complaints due to motility problems, to more impairing dysfunctions. Some 
patients have chronic diarrhoea, while others have constipation, spastic colon, laxity of the anal sphincter and faecal soiling. Among patients who have only minimal impairment of skeletal muscle function, gastro-intestinal problems can be the first real impairment and reason to seek medical attention.

Cardiac rhythm problems are another complication of myotonic dystrophy. Due to an abnormality in the conduction fibres of the heart, its electrical activity can be disrupted, leading to slow and/or irregular heart rhythm. Abnormalities of the electrocardiogram are frequent in myotonic dystrophy patients, but only a limited number of them have clinically significant cardiac problems. In the worst case, sudden death occurs. It is unclear how many premature deaths in myotonic dystrophy are due to cardiac arrhythmias.

Another frequent symptom of myotonic dystrophy is early balding, which is more pronounced in males than females. The combination of muscle wasting of the jaw and temporal muscles, lack of facial expression and ptosis of the eyelids, and early balding, give the typical facial appearance of the adult patient with myotonic dystrophy. Patients with myotonic dystrophy frequently have an otherwise seldom benign epithelioma of the hair follicles.

These clinical symptoms are the most prominent and well known. Other problems of myotonic dystrophy include endocrine dysfunctions, disorders of the immune system, of the liver, gallbladder and kidney and of the peripheral nerves. The endocrine dysfunctions consist of insulin resistance that may influence brain metabolism [Annane et al., 1998], abnormal secretion of growth hormone, testicular atrophy and others. Infertility is frequent in patients with myotonic dystrophy, particularly in males.

Behavioural changes in adult patients, due to involvement of the central nervous system, have been known since early research in myotonic dystrophy. In addition to daytime sleepiness, other cognitive and personality changes have been described [Harper, 2001]. These will be reviewed in section 5, this chapter. Myotonic dystrophy is a progressive degenerative disorder and, as such, problems become worse with age. Though the variability in symptoms and degree of impairment varies widely in myotonic dystrophy, the disease can be a cause of early death. The mean age at death in patients with the classic form of myotonic dystrophy is around 54 years [de Die-Smulders C.E. et al., 1998], the main causes of which are due to respiratory and cardiac problems. 
Table 6 Principal clinical manifestations in myotonic dystrophy.

\begin{tabular}{|c|c|}
\hline System affected & Symptoms* \\
\hline \multirow[t]{5}{*}{ Skeletal muscles } & $\begin{array}{l}\text { - Myotonia, in particular in adult and mild forms, absent in congenital } \\
\text { form. }\end{array}$ \\
\hline & $\begin{array}{l}\text { - Progressive wasting \& weakness, beginning in face, head and neck, } \\
\text { distal limb muscles, respiratory muscles }\end{array}$ \\
\hline & - Speech problems. \\
\hline & - In adult and mild form: myotonia. \\
\hline & - In congenital form: severe muscle hypotonia, clubfoot \\
\hline Gastrointestinal & $\begin{array}{l}\text { - Swallowing problems, oesophageal dysfunction, constipation, } \\
\text { megacolon, laxity of anal sphincter, diarrhoea, vague complaints due to } \\
\text { motility problems }\end{array}$ \\
\hline Heart & - Conduction problems leading to arrhythmia, cardiomyopathy \\
\hline Respiratory system & $\begin{array}{l}\text { - Weakness of respiratory muscles and poor regulation of respiratory } \\
\text { rhythm due to central nervous system problems; ensuing poor } \\
\text { ventilation and lung infections }\end{array}$ \\
\hline Endocrine & $\begin{array}{l}\text { - Testicular atrophy and infertility; insulin resistance with diabetes } \\
\text { mellitus }\end{array}$ \\
\hline Eye & $\begin{array}{l}\text { - Ptosis of the eyelids, cataract, slow reactions of the pupil muscles, slow } \\
\text { eye movements }\end{array}$ \\
\hline Skin & - Early balding, hair follicle tumors \\
\hline Immune system & - Reduced levels of immunoglobulins \\
\hline Peripheral nerve & - Polyneuropathy \\
\hline \multirow[t]{4}{*}{ Central nervous system } & $\begin{array}{l}\text { - Excessive daytime sleepiness, excessive nighttime sleep, apathy, } \\
\text { specific memory problems, possible personality changes }\end{array}$ \\
\hline & - Mental retardation in congenital myotonic dystrophy. \\
\hline & - Learning problems in childhood onset myotonic dystrophy. \\
\hline & - Other behaviour problems in children: social functioning, ADHD. \\
\hline
\end{tabular}

*It should be noted that the presence and degree of these symptoms vary widely (adapted from Thornton [1999]).

\subsection{The congenital type of myotonic dystrophy}

In 1960, Vanier gave the first description of myotonic dystrophy in infants [Vanier, 1960]. This was much later than the descriptions of the classical type of myotonic dystrophy in adults. Frequent neonatal deaths in families with myotonic dystrophy had previously probably not been recognized as children with a severe form of the disease. Alternatively, they may have been attributed to maternal problems due to myotonic dystrophy. Indeed, children with congenital myotonic dystrophy generally inherit the disorder from their mother (see section 
5.1, this chapter), while patients with the classical adult form may inherit it from either parent. Only when children with congenital myotonic dystrophy could be kept alive by means of improved neonatal resuscitation techniques did it become clear that a congenital form of myotonic dystrophy existed. Symptoms are already present before birth, as these children show reduced foetal movements. Neonates have severe muscle weakness, with potentially fatal feeding and respiratory problems. If they survive this initial phase of extreme hypotonia, muscle strength improves and the child will develop slowly. Muscle weakness is pronounced, but improves with age. Unlike patients with adult onset myotonic dystrophy, these children have no marked muscle wasting. Muscle weakness is particularly evident in the face, with an open mouth and tented upper lip as clinical hallmarks of congenital myotonic dystrophy. Motor development of these children is delayed, and many will need physiotherapy to learn to walk. A number of infants are born with clubfeet (talipes). Myotonia is markedly absent in the congenital form.

Cognitıve development in childaren with congenitai myotonic dystrophy is deiayed. They have an average mental age in the mildly retarded range (see section 5.1, this chapter). Due to peripheral muscle hypotonia and to developmental problems in the central nervous system, speech and language development are delayed [Harper, 2001].

As in the classic form of myotonic dystrophy, gastro-intestinal symptoms occur in early life. Other systemic features of the classical type of myotonic dystrophy may be present in patients with the congenital form. Longitudinal research looking at the various systemic problems in this form of myotonic dystrophy is scarce, and it is still unclear whether these symptoms are already present in early life, or whether they appear gradually in the course of development. Most children with congenital myotonic dystrophy generally live into adulthood. In adolescence and early adulthood, they have increasing muscle weakness and other characteristics of the classical adult type. Only half of them survive into their thirties and forties [Mathieu et al., 1999].

Though it is now evident that the classical adult form and the congenital form of myotonic dystrophy have the same genetic cause, they seem to belong to two different categories of disorders [Harper, 2001]. Adult myotonic dystrophy is a typical progressive degenerative disorder, where a formerly healthy individual becomes gradually more impaired. Congenital myotonic dystrophy however, has more characteristics of a developmental disorder, in which an individual with severe problems in early childhood improves progressively though he or she maintains a number of features of the disorder. Only in later life do individuals with congenital myotonic dystrophy show signs of degeneration. Despite the same genetic cause, it 
is still unclear whether different pathophysiological mechanisms account for the different characteristics in these two forms of myotonic dystrophy.

\subsection{Other phenotypes in myotonic dystrophy}

Although the classical adult type and the congenital type of myotonic dystrophy are best known and generally well recognized by physicians, two other forms exist: the mild form and the childhood or juvenile form (see Table 7). In the mild or minimal form of myotonic dystrophy, subjects have no or only minimal muscle weakness and myotonia. Symptoms begin after the age of 50 and are not easy to differentiate from normal senescence [Thornton, 1999]. Cataract of the eye lens at an earlier age may be considerable and sometimes this is the only reason why affected individuals seek medical attention. In these patients, the diagnosis of myotonic dystrophy is often made only after the diagnosis has been made in more affected relatives.

Table 7 The four subtypes of myotonic dystrophy

\begin{tabular}{lclc}
\hline Subtype & Age at onset & Initial symptoms & $\begin{array}{c}\text { Range of number } \\
\text { of CTG repeats }\end{array}$ \\
\hline Congenital & $\begin{array}{c}\text { Pre-natal/ } \\
\text { birth }\end{array}$ & $\begin{array}{l}\text { Reduced foetal movements; swallowing } \\
\text { and respiratory problems at birth, } \\
\text { hypotonia, }\end{array}$ & $1000-5000$ \\
$\begin{array}{l}\text { Childhood } \\
\text { (juvenile) }\end{array}$ & $1-12$ years & $\begin{array}{l}\text { Learning and/or behaviour difficulties; } \\
\text { speech problems }\end{array}$ & $500-2000$ \\
Adult (classical) & $12-50$ years & Myotonia, muscle weakness & $100-1000$ \\
Mild & $>50$ years & Cataract & $40-80$ \\
\hline
\end{tabular}

(adapted from de Die-Smulders [de Die-Smulders C., 2000])

In the childhood or juvenile type of myotonic dystrophy, children are unaffected at birth and show symptoms before the age of 12. Mild muscle signs develop in late childhood or adolescence, and are seldom impairing [de Die-Smulders C., 2000]. These children may have significant cognitive and/or behavioural problems that precede the muscle symptoms [Steyaert et al., 1997; Steyaert et al., 2000]. Medical attention is sought first for behaviour or learning difficulties, rather than for physical complaints. If the clinician is not aware that myotonic dystrophy occurs in the family, he or she may miss the fact that the presenting behaviour problems may be early symptoms of this disease. In adulthood, children with the juvenile form have progressive muscle weakness and the other system manifestations of myotonic dystrophy. Though these individuals have earlier signs of the disease and manifest 
earlier impairment, current research does not clarify whether these patients have an average shorter life expectancy than patients with the classical adult type [Mathieu et al., 1999].

\subsection{Other myotonies}

When the gene and the mutation for myotonic dystrophy were discovered on chromosome 19. the mutation could not be demonstrated in all affected families. Based on clinical differences, the phenotype in these families was called proximal myotonic myopathy (PROMM)[Moxley and Ricker, 1995; Ricker, 1999]. More recently, the ZNF9 gene with a related type of mutation was discovered on chromosome 3 in individuals with PROMM/DM2 [Liquori et al., 2001]. Based mainly on the different causal genes, a differentiation is made between myotonic dystrophy type 1 - with a mutation on chromosome 19, and the most frequent mutation (95\% of cases)-, and myotonic dystrophy type 2 (about $5 \%$ of cases), which is caused by a CCTG tetranucleotide repeat mutation in the ZNF9 gene on chromosome 3. It is unclear whether all cases of PROMM have in fact myotonic dystrophy type 2, or whether there is still another genetic cause. We will not consider PROMM and DM type 2 further, as little is known about central nervous system involvement in this disorder.

\section{The genetics of myotonic dystrophy}

Myotonic dystrophy is an autosomal dominant inherited disorder: a carrier of the mutation has a $50 \%$ risk of transmitting the mutant gene. A striking characteristic of families with myotonic dystrophy, is that older generations are only mildly affected and exhibit symptoms at an older age, while individuals in younger generations are more severely affected and present symptoms at an earlier age [Höweler et al., 1989]. As in some other inherited neuropsychiatric disorders, such as Huntington's chorea, the symptoms of myotonic dystrophy may worsen and manifest themselves at an earlier age in each consecutive generation. This phenomenon is called anticipation. The underlying mechanism is that of expanding polynucleotide repeats, or dynamic mutation [Richards R.I., 2001]. To understand this mechanism we have to look at the molecular genetics of myotonic dystrophy.

\subsection{Molecular genetics}

The mutation causing the disease was discovered in 1991 [Aslanidis et al., 1992; Buxton et al., 1992; Harley et al., 1992a]. The mutation is within a gene on chromosome 19 (19q13.3) that codes for an enzyme, the myotonic dystrophy protein kinase (DMPK). The precise 
function of this protein is not yet clear. An untranslated2 region of the gene contains an unstable repeat sequence of three base pairs: CTG. This locus is called the DM1-locus. In the general population the number of (CTG)n repeats varies between five and 37 repeats, and the same number of CTG repeats is inherited from generation to generation. In some families however, the number of CTG-repeats increases from one generation to the next. When a threshold of 50 repeats is reached, subjects may be mildly affected. The longer the expansion, the more unstable it becomes and may expand from one generation to the next. The increase in repeat number from one generation to the next and the moderate correlation between repeat number and the severity of the phenotype demonstrates the phenomenon of anticipation [Harper et al., 1992]. There is evidence that the repeat length increases more during maternal rather than paternal transmission [de Die-Smulders C., 2000; Lavedan et al., 1993]. Men with moderately long expansions are generally infertile, while women are often still fertile. Consequently, an increase from a moderate to a long expansion will almost exclusively occur after maternal inheritance. As longer expansions are often associated with the congenital phenotype, this phenotype will usually occur after maternal inheritance [Hageman et al., 1993].

Another characteristic of the mutation is that it is not only unstable from one generation to the next, but also within one individual. There is marked somatic mosaicism of the expansion length [Lavedan et al., 1993] and, at least in peripheral blood cells, the expansion length progresses with age [Martorell et al., 1998]. Consequently, the expansion length found in peripheral lymphocytes of an individual of a certain age, is not the same and most likely longer than that in brain cells.

More complicated than the genetics of myotonic dystrophy is the fact that the mutation occurs in an untranslated region of the gene, and that cells containing the mutation in the DMPKgene produce the intact protein, although probably in lower quantities. Several theories have been proposed, and are described elsewhere [Tapscott and Thornton, 2001]. What is important to know from the present findings is that different mechanisms may underlie different groups of symptoms. Some symptoms, like the cardiac rhythm problems, are likely caused by a limited availability of the gene product of the myotonic dystrophy gene, the DMPK-protein. There is evidence that other symptoms may be caused by the messenger RNA (mRNA)

\footnotetext{
${ }^{2}$ Transcription is the process occurring in the cell nucleus in which the DNA is copied into messenger RNA (mRNA). mRNA carries the genetic information from the cell nucleus to the cytoplasm of the cell. In the translation process, the mRNA is used as information in the building of proteins, the final product of genes. In the translation process, only parts of the mRNA are actually used. Some parts have no direct coding function, and though transcripted from DNA to mRNA, they will not be translated.
} 
transcribed from the mutant gene. This mRNA also contains a long expansion and cannot be processed as effectively as normal mRNA. It accumulates in the cell nucleus and may disrupt normal cell functions by perturbing the processing of other mRNAs such as those for ion channels [Kimura et al., 2000], insulin receptor [Savkur et al., 2001] and cardiac isoform of troponin T [Philips et al., 1998]. This is thought to cause the muscle weakness and wasting. Still other mechanisms may be responsible for the cataract of the eye, mediated through haploinsufficiency for the SLX5-gene [Klesert et al., 2000; Sarkar et al., 2000; Winchester et al., 1999]. It is currently not known which mechanism causes the dysfunctions of the central nervous system in myotonic dystrophy.

\subsection{Correlation between expansion length and clinical form of myotonic dystrophy}

Four clinical forms of myotonic dystrophy can be differentiated from each other (Table 7). In forms with an older age of onset, subjects have in average a shorter expansion length. This is a group effect, and there is significant overlap between the groups. At both ends of the range, there seems to be a threshold effects: subjects with congenital myotonic dystrophy always have more than 1000 CTG-repeats at the mutation site, while patients with the mild form have fewer than 80 CTG-repeats [Thornton, 1999]. For CTG repeats between 80 and 1000, the correlation between phenotype severity and number of repeats is lower. Severity, defined in terms of cognitive and neuromuscular impairment, correlates more with the age of onset than with the expansion length of the CTG-repeat. The same is true for life expectancy. Subjects with the congenital form have, on average, a shorter life expectancy than those with childhood or adult onset [Mathieu et al., 1999]. Here too, there is significant overlap between the groups. Subjects with the mild form probably have a normal life expectancy.

\section{Behavioural and cognitive aspects of myotonic dystrophy}

Early studies described behavioural anomalies in adult myotonic dystrophy patients [Harper, 2001]. Early researchers noted apathy and neglect as signs of myotonic dystrophy and stated that the myotonic patient's home could be identified through its neglected aspect (Caughey and Myrianthopoulos, 1963, cited in [Harper, 2001]). Lower intelligence, lack of initiative, and apathy, were considered as part of the disease, in studies matching myotonic dystrophy with other muscular dystrophies with comparable muscle impairment. Excessive daytime sleepiness has been frequently described in adult type myotonic dystrophy, and some studies suggest a higher incidence of depression and/or personality disorders in adult patients with 
myotonic dystrophy. Children with congenital myotonic dystrophy often present with a developmental delay.

These findings raise several questions. (1) Which behaviour characteristics are typical for the myotonic dystrophy phenotype, and which are rather anecdotal or coincidental? (2) Are the different subtypes of myotonic dystrophy associated with different behaviour problems? (3) Are the behavioural findings a consequence of brain involvement in myotonic dystrophy, or are they secondary to the muscle weakness and chronic disability that patients with myotonic dystrophy experience?

\subsection{Cognitive characteristics}

Early research on myotonic dystrophy suggested that the disease might be a cause of mental retardation [Calderon, 1966]. Larger studies in the 1980s and early 1990s confirmed the fact that, as a group, patients with myotonic dystrophy are cognitively impaired [Woodward et al., 1982]. The degree of cognitive delay varied from study to study. Bird and colleagues found that six out of 29 patients had IQ's below 70 [Bird et al., 1983]. Portwood [ 1986] studied 43 patients and found significant intellectual impairment in the subgroup of patients with maternal inheritance, but not in the patients with paternal inheritance. Sinforiani [1991] confirmed this later. Bird [1983] and Franzese [1991] found females to be intellectually more impaired than males, though others did not confirm this. Degree of intellectual impairment was found to correlate with age of onset [Huber et al., 1989]. All these studies were performed before the genetic mechanism of myotonic dystrophy had been revealed, which made the findings that intellectual impairment correlated with age of onset and with maternal inheritance difficult to understand.

After the discovery of the dynamic mutation in myotonic dystrophy, studies on intellectual impairment could be correlated with the length of the CTG expansion. Turnpenny [1994] and later Perini [1999] found that longer expansion lengths at the myotonic dystrophy mutation locus correlate moderately with the degree of intellectual impairment. This is different from what is seen in Fragile $\mathrm{X}$ syndrome. In Fragile $\mathrm{X}$ syndrome there is a sharp threshold phenomenon between the premutation and the full mutation. In myotonic dystrophy, the variance is large, and the expansion length does not predict individual functioning accurately. In Turnpenny's study, younger age of onset correlated more strongly with lower intelligence than expansion length. However, as mentioned in the section on molecular genetics, there are important problems in correlating the expansion length in peripheral blood cells and in brain cells in a group of individuals with a wide age range: there is a continuous growth of the 
repeat with increasing age, and there is a significant somatic mosaicism [Martorell et al., 1998].

The level of cognitive impairment seems to be directly related to age of onset and repeat length, but not to muscular impairment. There is broad consensus that children with congenital myotonic dystrophy have the lowest IQs [Aicardi et al., 1975; Harper, 1975; Huber et al., 1989; Johnson E.R. et al., 1995; Roig et al., 1994; Steyaert et al., 1997; Tuikka et al., 1993]. At least $75 \%$ of them have IQs below 70 . Most are in the mildly retarded range, but some children have a moderate to severe mental retardation. Theoretically, their cognitive impairment might be caused by a direct effect of the mutation on the brain, or the effect of muscular impairment in infancy on psychomotor development. Subjects with juvenile-onset myotonic dystrophy already have cognitive impairment before they have significant muscular complaints [Goossens et al., 2000; Steyaert et al., 1997]. On the other hand, cognitive impairment in adult-onset myotonic dystrophy is generally very mild [Franzese et al., 1991] or even absent [Tuikka et al., 1993].

Data on possible cognitive deterioration during the course of the disease are limited and equivocal. Tuikka [1993] found no cognitive decline in sixteen myotonic dystrophy patients after an average follow-up time of 12 years. In a study of 16 children and adolescents with congenital or juvenile onset myotonic dystrophy, reliable two year follow-up data were available for two subjects and both showed a significant decline on the same IQ-test, the Wechsler Intelligence Scale for Children Revised [Steyaert et al., 1997]. Possible cognitive deterioration in myotonic dystrophy is certainly a subject of further research, particularly in children with the congenital and juvenile form of the disease.

Another area where further research is necessary is whether there is only a global cognitive impairment in some forms of myotonic dystrophy, or whether more specific cognitive problems can be observed. Most studies have not found significant differences between verbal and performance intelligence. Palmer et al. [1994] noted lower intelligence, visualconstructive and executive function problems in patients with maternal inheritance but not in those with paternal inheritance. Memory and visual-perception skills were normal in both groups. It should be noted that individuals with maternal inheritance will more often present with congenital myotonic dystrophy, and thus be more affected as a group. A more recent study with a homogeneous group of non-congenital onset patients, all with normal intelligence, showed that these persons do have specific memory impairment [Rubinsztein et al., 1997]. In this group, there was a trend towards impairment of executive function as measured by the Wisconsin Card Sorting Test. Earlier, Woodward and colleagues found more 
severe impairment of executive function in a group of 17 myotonic dystrophy patients [Woodward et al., 1982]. In their study, subjects with myotonic dystrophy scored worse than controls on almost every neuropsychological measure, and the finding of executive function impairment was not specific. A study by the Maastricht-Leuven research group demonstrated Attention Deficit Hyperactivity Disorder (ADHD) in 4 of 16 children with congenital and juvenile myotonic dystrophy (Chapter 9) [Steyaert et al., 1997], which is significantly higher than the frequency of $3-5 \%$ ADHD occurring in the school-age children's population [American Psychiatric Association, 1994]. These data were confirmed in a second study limited to children with juvenile onset myotonic dystrophy, where 8 in 24 children were given this diagnosis [Goossens et al., 2000]. As the pattern of neuropsychological findings in children with ADHD implicates deficits in executive function [Faraone and Biederman, 1998; Sergeant, 2000], the finding of ADHD in children with myotonic dystrophy probably reflects deficits in executive function in these children.

\subsection{Somnolence, hypersomnia and sleep apnoea.}

In early observations, the somnolence or excessive daytime sleepiness of individuals with myotonic dystrophy may have been seen as a form of fatigue or as a consequence of respiratory depression due to muscle weakness [Harper, 2001]. The symptom is perhaps one of the most common features of myotonic dystrophy, and occurs typically when attention is not focused. Somnolence in myotonic dystrophy is independent of irregular breathing during night sleep and sleep apnoea, which may also occur in myotonic dystrophy [Gilmartin et al., 1991; van der Meche et al., 1994; Ververs et al., 1996]. The symptom is significantly more frequent in subjects with myotonic dystrophy than in controls with other neuromuscular disorders [Phillips et al., 1999; Rubinsztein et al., 1998]. Rubinsztein et al. [1998] showed that somnolence is independent of apathy and of depression in patients with myotonic dystrophy. They conclude that it likely reflects central nervous system involvement and is not secondary to other symptoms. Phillips et al. [1999] showed that daytime somnolence in myotonic dystrophy is partially correlated with the degree of muscular disability.

Several authors report that adult patients with myotonic dystrophy also have excessive nighttime sleep [Ono et al., 1995; Ono et al., 1998; Rubinsztein et al., 1998]. The same observation was made in children with juvenile myotonic dystrophy [de Die-Smulders C., 2000; Steyaert et al., 1997]. 


\subsection{Apathy}

Apathy, lack of initiative, sitting idly, and indifference have been reported since the early observations on myotonic dystrophy [Harper, 2001]. Systematic observations on apathy in myotonic dystrophy have been performed by Ambrosini and Nurnberg [1979] and more recently by Rubinsztein et al. [1998]. The latter study could elegantly demonstrate that apathy is a common and independent feature of myotonic dystrophy, and cannot be accounted for by depression or muscle weakness. It is, therefore, likely to reflect central nervous system involvement. One possible consequence of apathy in myotonic dystrophy may be the high level of unemployment found among patients with mild physical disability [Fowler et al., 1997].

\subsection{Personality changes}

A more controversial subject is whether individuals with myotonic dystrophy have a particular personality profile, or a higher frequency of personality disorders. Based on personal impressions of their patients, several early researchers on myotonic dystrophy found unusual personality characteristics [Harper, 2001]. However, their impressions were not all the same. Some authors found myotonic dystrophy patients to be cheerful and careless, others saw them as hostile and unreliable. Research on personality problems has become more objective, but even then, the subject remains unclear in myotonic dystrophy. Using the Minnesota Multiphasic Personality Inventory, Bird [1983] found significant personality changes in eight of $25(32 \%)$ subjects with myotonic dystrophy. On the other hand, Franzese [1991] did not find significant personality traits in a group of 28 patients with juvenile or adult-onset myotonic dystrophy. Palmer et al. [1994] found a high incidence of "dependent tendencies" in the personality of myotonic dystrophy subjects, and attributed this to their adjustment to a disabling disorder. In another study, Delaporte et al. [1998] found a homogeneous personality profile in the group of myotonic dystrophy subjects they studied. They used the International Personality Disorder Examination in adults with minimal muscle weakness, a group of healthy controls and a group of controls with facioscapulohumeral dystrophy. Most of the 15 subjects with myotonic dystrophy displayed traits of avoidant personality, four of whom exhibited avoidant personality disorder. They concluded that a profile consisting of avoidant, obsessive-compulsive, passive-aggressive and schizotypic traits could not be attributed to adjustment to the disease, but reflected central nervous system involvement in myotonic dystrophy. 
In these different studies age of onset, degree of physical disability, research instruments and definition of personality changes vary widely. It is thus impossible to compare them accurately. However, most researchers and clinicians agree that many persons with myotonic dystrophy have "something" in the area of emotional functioning and interpersonal relationships, which is not observed in patients with comparable degree of muscle weakness due to other progressive neuromuscular diseases. It remains a challenge to identify this something, perhaps because classical personality inventories that were used in these studies are not suitable to detect personality traits that mainly occur in organic personality changes.

\subsection{Depression}

Several studies have suggested that signs of depression are found more frequently in DM patients. Duveneck [1986] described significantly more depressive tendencies in patients with myotonic dystrophy than in patients with other non-progressive or progressive neuromuscular disorders. However, the degree of depressive traits was mild and the authors could not conclude that depressive disorder occurs more frequently in myotonic dystrophy. Another study found depressive disorder in $17 \%$ of myotonic dystrophy patients with severe muscle impairment [Colombo et al., 1992]. Less severely affected individuals were not found to be more severely or more frequently depressed than normal controls [Bungener et al., 1998; Rubinsztein et al., 1998]. To the contrary, Bungener [1998], found flat affect among persons with myotonic dystrophy. It is possible that some symptoms like flat affect, apathy, somnolence (see section 5, this chapter) and other characteristics of myotonic dystrophy may give the wrong impression that mildly affected myotonic dystrophy patients appear to be depressed. On the other hand, one can imagine that the threat of further physical decline or even sudden death due to cardiac arrhythmia can cause depressive disorder in already severely affected patients. The risk of transmitting the disease to their children is another severe stressor for parents with myotonic dystrophy [Faulkner and Kingston, 1998], and this might also affect these individuals' mood. The question as to whether patients with myotonic dystrophy have a higher incidence of depressive disorder; and, if so, whether this reflects central nervous system involvement or adjustment problems in having or transmitting the disorder, remains to be answered.

\subsection{Child psychiatric findings in myotonic dystrophy}

The congenital and juvenile forms of myotonic dystrophy have been described more recently than the classical adult-onset form. To our knowledge, research on the behaviour phenotype in these forms of myotonic dystrophy is limited, apart from the consistent finding that children 
with congenital myotonic dystrophy have cognitive developmental delays, with an average in the mildly retarded range (see section 5.1, this chapter). Although children with congenital myotonic dystrophy initially present with neuromuscular problems and developmental delay, we observed that the first complaints in children with juvenile onset myotonic dystrophy symptoms occur before age 12, but are absent at birth - were often learning and attention problems, even in children with IQs in the low-normal range [Goossens et al., 2000; Steyaert et al., 1997] (Chapters $9 \&$ 10, this thesis). Using the Child Behavior Checklist, we found a marked increase of attention deficits and social problems in children with juvenile myotonic dystrophy, all of which had no or minimal muscle weakness. A standardized child psychiatric interview showed a higher than expected frequency of Attention Deficit Hyperactivity Disorder: 4 in 16 in the first study [Steyaert et al., 1997], 8 in 24 in the second study [Goossens et al., 2000]. Symptoms of lack of impulse inhibition and of attention deficit were more pronounced in these children than symptoms of hyperactivity. Both studies also showed an increase of anxiety disorders in children with juvenile myotonic dystrophy.

These studies do not demonstrate convincingly whether the behaviour problems and child psychiatric disorders were a direct consequence of central nervous system involvement, or of adjustment problems to the disease. The latter hypothesis seems unlikely for several reasons. In most subjects, the behaviour problems existed before any neuromuscular sign of the disease, and the very significant increase of one particular child psychiatric disorder, Attention Deficit Hyperactivity Disorder, rather suggests a brain involvement. Indeed, adjustment problems would most likely be transient, more diverse, and in line with the personality development of the individual subjects.

\section{Brain involvement in myotonic dystrophy}

Individuals with neuromuscular disorders with a genetic origin may show behavioural problems. These problems however, should not automatically be attributed to a direct effect of the mutant gene on the brain. Having a chronic disability may cause emotional and mood problems in affected individuals, e.g. individuals with diabetes mellitus have higher levels of anxiety and mood problems than controls [Kohen et al., 1998]. The idea of having transmitted the mutated gene to one's children may add to the stress, as observed in otherwise healthy mothers of children with fragile-X syndrome [Franke et al., 1996; McConkie-Rosell et al., 1999]. In children with congenital myotonic dystrophy, the severe breathing problems at birth might lead to brain damage and the muscle weakness in early childhood might impede the child's normal development. These would be indirect mechanisms influencing the behaviour 
phenotype. In myotonic dystrophy research, many resources have been used to demonstrate direct effects of the mutated gene on the brain. However, questions remain open concerning the importance of indirect effects of this disabling disorder on development and behaviour. Insulin resistance is prominent in myotonic dystrophy patients, and impairs cerebral glucose metabolism [Annane et al., 1998] .

Various approaches have been used to demonstrate direct effects. One has been to take subjects with other forms of neuromuscular impairment as control subjects in behaviour studies with myotonic dystrophy patients. Facioscapulohumeral dystrophy and CharcotMarie-Tooth disease are other hereditary neuromuscular disorders that begin in late childhood or adulthood, with slowly progressive disability. The degree of disability is very similar to that in myotonic dystrophy. This approach demonstrates that personality problems [Delaporte, 1998], relative underemployment [Fowler et al., 1997], apathy and hypersomnia [Rubinsztein et al., 1998] are specifically associated with myotonic dystrophy, and not with having a neuromuscular disorder. These findings support the idea that myotonic dystrophy is not only a neuromuscular disorder, but that the brain is also directly involved.

A second and more fundamental approach has been to examine the brains of patients with myotonic dystrophy. This approach has provided convincing evidence that myotonic dystrophy is a brain disorder, as much as a neuromuscular disorder [Ashizawa, 1998]. Neuropathological studies in adult onset myotonic dystrophy have shown several neurodegenerative changes in the brain: cell loss, neuronal inclusion bodies, neurofibrillary tangles, and others [Harper, 2001]. These changes are somewhat different from those found in Alzheimer's disease, and thus can be directly related to myotonic dystrophy. Neuropathological findings in congenital myotonic dystrophy are less conclusive, though neuronal migration problems have been found in a few cases.

Many brain-imaging studies have been performed in myotonic dystrophy. The earliest ones, using air encephalogram, showed enlarged cerebral ventricles [Refsum et al., 1967]. This finding was reproduced in later studies with magnetic resonance imaging (MRI) [Glantz et al., 1988; Huber et al., 1989]. In some patients, cortical atrophy was also demonstrated [Censori et al., 1994]. Although some studies showed that cognitive impairment correlated with degree of cerebral atrophy [Huber et al., 1989], others did not confirm this finding [Censori et al., 1994]. An important problem in these equivocal results is that the different forms of myotonic dystrophy had not been clearly delineated in these studies. Hashimoto et al. compared MRI findings in congenital versus adult onset myotonic dystrophy and demonstrated that the cerebral atrophy is more marked in the congenital form [Hashimoto et al., 1995]. Giubilei et 
al. found that atrophy of the anterior part of the corpus callosum correlates with sleep breathing irregularities [Giubilei et al., 1999].

Another common MRI finding in myotonic dystrophy is the presence of white matter hyperintense lesions [Damian et al., 1994b; Di Costanzo et al., 2001; Hashimoto et al., 1995; Sinforiani et al., 1991]. Damian et al. found that these white matter lesions correlate with cognitive impairment when they lie in the white matter immediately adjacent to cortex, but had no clear clinical significance when they have a periventricular localization [Damian et al., 1994b]. Neurocognitive dysfunctions correlate more strongly with white matter anomalies than with ventricular enlargement [Abe et al., 1994]. Chang et al. [1993] found evidence of diminished cerebral blood flow, most severe in the frontal and temporoparietal association cortex. Functional Magnetic Resonance Imaging (FMRI) is a non-invasive imaging technique that makes it possible to study the concentration of specific chemical compounds in the brain of living patients. Using FMRI, Chang et al. [1998] demonstrated changed concentrations of several chemical compounds in the brain of patients with myotonic dystrophy. The degree of changes correlated well with the length of the CTG-expansion in the myotonic dystrophy gene.

Brain imaging studies are subject to the same limitation as psychological investigations, which is that different researchers have included different subgroups of the broad spectrum of myotonic dystrophy phenotypes, thus making comparisons across investigations difficult. Nonetheless, the neuropathological studies, and the more recent brain imaging studies, give convincing evidence that the brain is involved in myotonic dystrophy and support the view that at least a substantial part of the behaviour phenotype is the direct consequence of a brain disorder. However, elucidating the pathophysiological mechanisms between the molecular changes in myotonic dystrophy, the anatomical and functional signs of brain involvement, and the behavioural phenotype, still requires a lot of work.

\section{Diagnosis of myotonic dystrophy}

Until the discovery of the gene and the molecular causes of myotonic dystrophy, the diagnosis was based on clinical observations and family history, and demonstrating electromyographical and pathological changes in the muscles [Harper, 2001]. Now that direct detection of the mutated gene through a DNA-test has become possible, making the diagnosis has become easier. This will probably lead to making the diagnosis in unaffected, or only minimally affected, family members of patients with myotonic dystrophy. This raises ethical questions, in particular if parents request asymptomatic children to be tested. The ethical consideration 
about paediatric genetic testing is that if children who are potential carriers of a disease of which they have no signs (yet), and if uncertainty about their carriership has no effect on later prognosis, these children should not be tested for the disease until they are mature enough to decide themselves whether they want to be tested. On the other hand, one should consider that in children, myotonic dystrophy may begin with behavioural rather than neuromuscular symptoms, and that lack of the latter symptoms should not impede making an early diagnosis. Another possible side effect of the easy and very reliable DNA-test is the fact that parts of the clinical assessment may be neglected in these patients with a complex multi-system disease.

\section{Conclusions}

Myotonic dystrophy is a multi-system disease with a wide spectrum of clinical manifestations. Depending on the widely varying age of onset and degree of impairment, four forms of myotonic dystrophy can be distinguished. The congenital form may present (initially) as a developmental disorder, with severe symptoms in infancy and consequent improvement. The other three forms present as a slowly progressive degenerative disorder, which is also the fate in adulthood of patients with congenital myotonic dystrophy. There is convincing evidence that the brain is directly affected, contrarily to many other neuromuscular disorders. The path from mutant gene to brain pathology, neurophysiological changes, and finally to behaviour is still unclear.

The behavioural phenotype is quite characteristic, but its degree of expression is variable. As a rule, an early age of onset of the disorder correlates with a more severe behavioural phenotype. There is a weaker correlation between age of onset and length of the CTGexpansion at the mutation site in the gene.

Mild mental retardation is common in congenital myotonic dystrophy, and milder learning problems are frequent in juvenile myotonic dystrophy. Often, they even precede neuromuscular symptoms in these children, and should thus warrant the possibility of myotonic dystrophy in children with learning problems in myotonic dystrophy families. It is equivocal whether adult-onset patients as a group have a lower intelligence, and there is a marked lack of follow-up data on the evolution of intelligence in all forms of myotonic dystrophy.

Besides these global impairments in cognitive ability, impairment of executive functions is present in all forms of myotonic dystrophy, but not in all individuals with the disease. In children with juvenile onset, deficits of attention and impulse control may be signs of poor executive functioning. Apathy is a very common symptom in myotonic dystrophy and is 
independent of sleep anomalies and depression [Rubinsztein et al., 1998]. In neuropsychological literature, apathy is mentioned as a sign of frontal lobe dysfunction and as such may correlate with poor executive function [Kolb and Whishaw, 1996]. Specific memory dysfunctions have been found in adult-onset myotonic dystrophy. Somnolence (excessive daytime sleepiness) and hypersomnia are frequent symptoms in myotonic dystrophy, and reflect brain involvement rather than fatigue due to muscle weakness.

It is equivocal whether myotonic dystrophy patients have a higher incidence of depressive disorders, and if so, what the pathological mechanism is. Some researchers have found a higher prevalence of particular personality traits, and/or personality disorders in myotonic dystrophy, while others have not. It is not clear whether these possible personality problems are independent of the apathy, poor executive functions and other neurocognitive signs that we discussed above.

Finally, to our knowledge, nothing is known about the behavioural phenotype in patients with the mild, late-onset form of myotonic dystrophy. Considering the reflections on age of onset and degree of behavioural impairment, it is most likely that these patients have no or only minimal behavioural characteristics. 

Part II. Studies in Fragile $X$ SYNDROME AND IN MYOTONIC DYSTROPHY 



\section{Chapter 6 Cognitive Profile in Adult, Normal Intelligent Female Fragile $X$ Carriers}

Steyaert J, Borghgraef M, Gaulthier C, Fryns JP, Van den Berghe H. (1992)

Centre for Human Genetics, University of Leuven, Belgium 


\section{Abstract}

Here we present the results of the study of the cognitive profile of 11 adult, normally intelligent female fragile $\mathrm{X}$ carriers. In all individuals, three types of testing were performed: full scale Wechsler Adult Intelligence Scale, a set of neuropsychological tests and an arithmetic achievement test.

All II subjects showed an average to above-average intelligence but 10 of 11 performed better on the performance than on the verbal subscale. Neuropsychological tests suggest a dysfunction of the reticulo-thalamic axis with deficiency on tests requiring visual memory and a pronounced deficiency of attention skills combined with an impulsive way of completing tests. 


\section{Introduction}

During the past few years an increasing number of studies have dealt with the psychological and behavioural profile of fragile X males [Borghgraef, 1991], [Curfs et al., 1989]. However, few studies have been reported on the intellectual and cognitive functioning of fra $(\mathbf{X})$ carrier females.

\section{Materials and methods}

We tested 11 women, all obligate carriers of the fragile $\mathrm{X}$ syndrome through the female line, age between 24 and 36 years. All had a normal phenotype. Fragile X screening was low (less than $4 \%$ fra $(\mathrm{X})$ cells in 4 , and negative in the 7 others). Full-scale Wechsler Adult Intelligence Scale (WAIS) testing was done and the same sequence of subtests was applied in all subjects.

The second part of the test setting was a set of neuropsychological tests covering a wide range of cognitive functions, all tested with the same sequence. The Bourdon-Wiersma test consists of a widely used cancellation task, evaluating continuous and focussed attention. Verbal memory and verbal learning were evaluated with Rey's 15- words test; auditory memory performance for digits with the digit subtest of Elizur's Test for Psycho-organicity; ability to structure and reproduce visual material with Rey's Complex Figure-test. The Trail-Making test was taken to evaluate the ability of the subjects to alternate different "mental tracks". Stroop's Colour Word test was taken to evaluate the ability to suppress interfering stimuli. Specific arithmetic disabilities were tested with an arithmetic achievement test evaluating the four basic arithmetic operations separately. This test is a Dutch school achievement test designed to evaluate fourth and fifth grade performance of Belgian school children, i.e. the level of arithmetics that school children should attain to succeed in vocational secondary schools and corresponding to the level which might have been expected from the tested subjects, taking into account their general educational level ( 7 vocational school, 2 technical school, and 2 non-university high-school).

\section{Results}

\subsection{Intelligence}

The mean intelligence quotient (IQ) of the 11 women was: verbal IQ (VIQ) $111.6(\mathrm{sd}=9.3)$. mean performance IQ (PIQ) 120.0 (sd=9.9), and mean full scale IQ (FSIQ) 116.5 (sd=9.1). 
Ten of the 11 subjects had a higher PIQ than VIQ. The mean group difference VIQ-PIQ was significant: $-8.4(\mathrm{p}=0.016)$.

In the subtest analysis we tried to circumvent the problem of averaging a subtest when the subjects have different IQ's, as this would automatically lead to between-subject differences. Hence, before averaging, we compared within-subject deviation of separate subtests. First, the average standard score was calculated in each subject, for the verbal and performance scale separately. Then, the difference between a particular subtest and the individual subscale average was calculated. Finally, these differences for each subtest separately were averaged for the group (Table 8), t-Values were calculated for the mean deviation, which should be zero for a group, considering the construction of the Wechsler-scales and their standard scores.

In the verbal subscale, the subtest "comprehension" was significantly higher than expected for the VIQ, while "arithmetic" was significantly lower then expected. In the performance subscale, we found that the subjects scored lower than expected on the subtest "object assembly".

Table 8 Mean deviation of mean Standard Scores for WAIS subtests.

\begin{tabular}{lccc}
\hline Subtest & $\begin{array}{c}\text { Mean score for the } \\
\text { group }\end{array}$ & $\begin{array}{c}\text { Mean deviation from individual } \\
\text { mean standard score }\end{array}$ & T-score \\
\hline Verbal Subscale & & -0.09 & 0.854 \\
Information & 6.45 & 1.18 & $4.64^{*}$ \\
Comprehension & 7.27 & -0.91 & $-2.86^{*}$ \\
Arithmetic & 5.64 & 0.27 & 0.65 \\
Similarities & 6.82 & -0.36 & -1.22 \\
Digit Span & 6.18 & -0.09 & -0.38 \\
Vocabulary & 6.45 & & 1.08 \\
\hline Performance subscale & & & 0.46 \\
Coding & & 0.37 & 0.98 \\
Picture Completion & 7.54 & 0.18 & 1.20 \\
Block design & 7.36 & 0.27 & $-2.56^{*}$ \\
Picture arrangement & 7.45 & 0.55 & \\
Object assembly & 7.73 & -1.36 & \\
\hline
\end{tabular}

For 11 subjects $(d f,=10), p<0.05$ when $t$-value $>2.28$ or $t$-value $<2.28$ (marked with $*)$. 


\subsection{Neuropsychological}

Since performances on many neuropsychological tests do not follow a normal distribution, we decided not to use the standard deviation normally applied in the statistical analysis of these tests. We rather used distribution-free methods i.e. the signed test around the median, or tests for proportions. In the latter, we compared the probability that the results might be distributed as they are in the test group, regarding the distribution in the normative population. The compared distributions are proportions (U-values at statistical analysis) around the raw score of a particular centile rank (centile 25) or cut-off score in the normative population.

Subjects had problems performing Rey's Complex Figure Test. In this test the score "structuration type" applies to the strategy the subject follows to copy the figure, while score "details " is an evaluation of the number and the position of particular details in the subject's drawing. The norms applied for the copy are more difficult than the norms applied for the memory reproductions. This group of subjects performed poorly on both "structuration type" and "details" when copying the figure. This effect was less pronounced at the memory reproduction. This pattern of performances at Rey's Complex Figure Test is frequently observed in older children with attention deficits and poor cognitive strategies.

That attention skills were poorer in these 11 adult fragile $\mathrm{X}$ carrier females than in the average population was confirmed in the results of Bourdon's Cancellation Task: 4 subjects went through the test with more important variation of speed than allowed, 8 subjects had more omissions than the cut-off score. Subjects performed well on tests that required only visual skills and orientation. We did not interpret the fact that 9 out of 11 subjects had one indicative error on Bender's Visual Gestalt Test as a problem of visual skills, as almost all subjects made the same error: in the Bender Test drawings that have dots, they drew little circles instead. The subjects did this as well drawing with a soft pencil as with an ink marker. The testing psychologist thought this phenomenon was rather due to the subjects' impulsive way of drawing than to a lack of visual skills.

The subjects performed well on auditory memory tasks, as well with digits as with unrelated words (Rey's 15 Words Test). However, they performed poorly on a visual memory task with non-complex nonsense drawings (Rey's 15 Figure Test).

As there was no well-standardized Dutch test that could evaluate verbal skills at the limits of normality, and as there is no overt aphasia in the subjects, we did not evaluate the verbal skills of the subjects through separate tests. However, clinical observations suggest a discrepancy 
between the quite high verbal fluency of most of the subjects, and the rather poor speech content in several of them. Taking into account the above- average intelligence of the subjects, this observation needs further investigation.

\subsection{Arithmetic test}

All subjects performed well above median percentiles on the four subtests of the arithmetic achievement test that was used: addition, subtraction, multiplication and division. However, it is appropriate to use this test rather for adults with average, or slightly below average intelligence and vocational school education. Although these criteria probably would apply for most female fragile $\mathrm{X}$ carriers, they do not apply for the present group of above average subjects. Thus, we consider the results of this test as rather inconclusive.

\section{Discussion}

The aim of the present study was to obtain data on the cognitive profile in fragile $\mathrm{X}$ carrier females. As the data and results of neuropsychological tests are only reliable in normally intelligent individuals, we therefore selected a group of 11 women with normal intelligence, belonging to eight different fragile $\mathrm{X}$ families diagnosed at the Centre for Human Genetics in Leuven. All were obligate fragile $X$ carriers through their mothers and had at least one affected son and one affected male relative in the maternal line. Females who inherited the fragile $X$ from their normal transmitting father were not included and will be the subject of a later study. It is well known that daughters from normal transmitting males constitute a separate subgroup of fragile $\mathrm{X}$ carriers without apparent effect of the carrier state on their phenotype and their intellectual functioning and, therefore, possibly also on their cognitive profile [Fryns J.P., 1989].

The results of the Wechsler Adult Intelligence Scale tests showed an average to aboveaverage performance in all subjects: all but one subject performed better on the performal than on the verbal subscale. The subjects performed better than normal on the subtest "comprehension" which indicates that their intelligence was not overrated. They performed significantly below average on the subtest "arithmetics" and on the "object assembly" subtest. However, is it premature to conclude that arithmetic skills are necessarily abnormally poor in fragile X carriers: the subtest "arithmetic" not only requires arithmetic skills, but also good attention skills as the task is given orally and in several items the data and required operation are embedded as a story, and, as is shown in the neuropsychological tests, precisely the attention skill of these women was well below expected values. 
The neuropsychological tests show a deficiency on tests that require visual memory. Furthermore, there was a pronounced deficiency on tests requiring attention skills: Bourdon's Cancellation. Task and Rey's Complex Figure Test. In addition, we noted the impulsive way the subjects worked at most tasks, which is, for instance, reflected in the very poor "structuration type" at copying Rey's Complex Figure Test and the rude, although geometrically correct drawings in most tests e.g. the plain circles instead of dots in Bender's Gestalt Test

This combination of poor attention skills and an impulsive way of completing tests is similar to that found in fragile $\mathrm{X}$ boys, who are in addition hyperactive. We may conclude that the present group of normally intelligent female fragile $\mathrm{X}$ carriers have similar cognitive profiles as affected fragile X boys, although they show it rather as a "trait" than as gross abnormality. It should be underscored that we do not know objectively whether this trait affects the general functioning of these women. Nevertheless, we have the strong impression that most of the tested subjects function below what might be expected for their intelligence level, and moreover, we noted that more than half of them show rather chaotic thinking. These observations suggest deficient executive functions. 


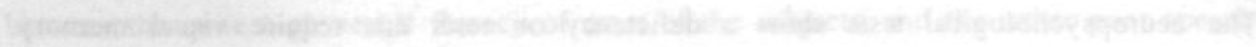

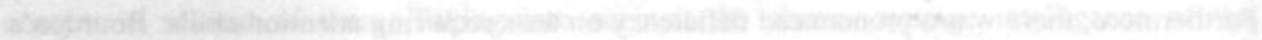

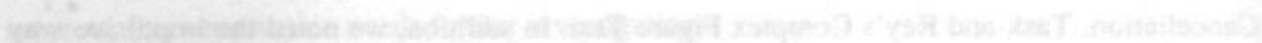

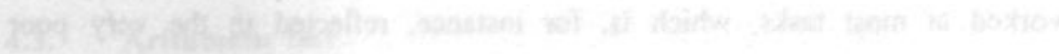

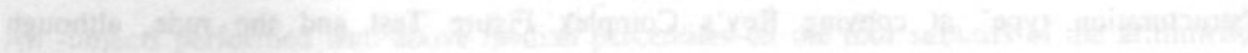

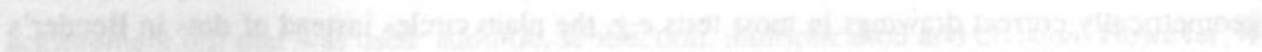

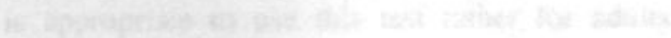

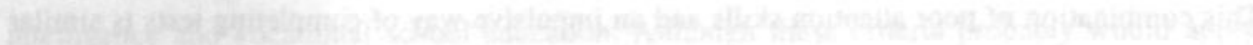

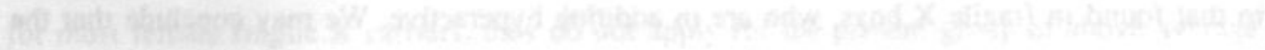

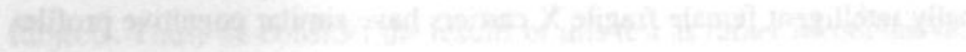

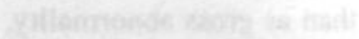

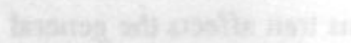

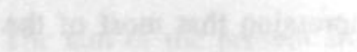

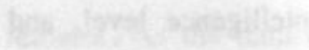

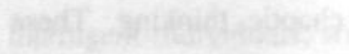

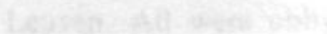

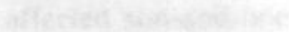

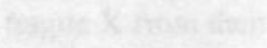

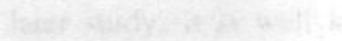

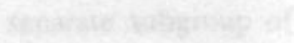

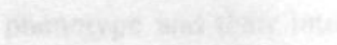

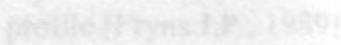

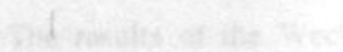

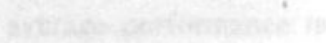

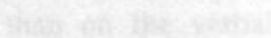

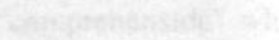

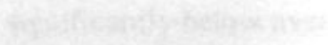

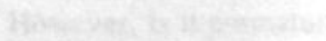

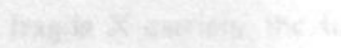

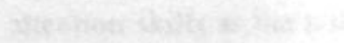

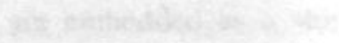

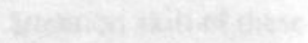




\section{Chapter 7 Molecular-Intelligence Correlation in Young Fragile X Males With a Mild CGG Repeat Expansion in the FMR1- Gene}

Jean Steyaert', Martine Borghgraef ${ }^{2}$, Eric Legius ${ }^{2}$, and Jean-Pierre Fryns $^{2}$ (1996).

' Centre for Clinical Genetics, University of Maastricht, the Netherlands.

${ }^{2}$ Centre for Human Genetics, University Hospital Gasthuisberg, Leuven, Belgium. 


\section{Abstract}

Several mechanisms can explain the occurrence of full-mutation fragile $\mathrm{X}$ males with an IQ level above -2 SD below mean, also called "high-functioning fragile $\mathrm{X}$ males." Incomplete methylation of the CpG island at the 5' end of the FMRI gene is one of these mechanisms. The present study describes the physical and behaviour phenotypes in seven fragile $\mathrm{X}$ boys with CGG repeat insertions in the FMRI gene between $600-2,400$ base pairs. The degree of methylation at the FMRI-associated CpG island ranges in peripheral blood lymphocytes from $0-95 \%$. Subjects with a low degree of methylation at this site have mild or absent physical characteristics of the fragile $\mathrm{X}$ syndrome, while subjects with a high degree of methylation at this site have more severe physical characteristics. In this range of CGG repeat insertion (6002,400 base pairs), the degree of methylation at the FMRI-associated CpG island is a good predictor of intelligence, while CGG repeat insertion length is not. 


\section{Introduction}

In the first descriptions of fragile $\mathrm{X}$ syndrome, mental retardation was considered an absolute characteristic of fragile $\mathrm{X}$ hemizygotes. Later, transmitting males were found in whom mental retardation and other characteristics of fragile $\mathrm{X}$ phenotype were absent, i.e., the so- called normal transmitting males [Daker, 1983], [Sherman et al., 1985]. After the molecular basis of the fragile X mutation was described [Verkerk et al., 1991], the normal transmitting males were found to have a shorter CGG-repeat amplification at the FMRI-gene, and the differentiation was made between a premutation, with an insertion of $<600$ base pairs, and a full mutation, with a longer insertion. Most males with a full mutation in the FMRI-gene have fragile X syndrome. Besides physical characteristics and often characteristic behaviour traits, the fragile $\mathrm{X}$ phenotype includes mild, moderate, or even severe mental retardation [Borghgraef et al., 1987].

Only recently, fragile $\mathrm{X}$ males with a full mutation have been described with an intelligence level in the so- called borderline or even normal range [Hagerman R.J. et al., 1994], [Rousseau et al., 1994b]. These IQ ranges are defined as between -2 and -1 SD below mean for borderline range, and IQ higher than -1 SD below mean for normal range. Hagerman [Hagerman R.J. et al., 1994] found mild physical signs of fragile X syndrome in most of these males. Several mechanisms may explain this phenomenon. First, intelligence is a characteristic influenced by many genes. Thus, as in any population, in a population of fragile $\mathrm{X}$ males the distribution of intelligence level follows a bell-shaped curve. However, in this particular population, the mean is displaced to the left and is around an IQ of 40, instead of IQ 100 , as in the total population. A sporadic individual with a complete FMRI-mutation could be at the far right end of the fragile $\mathrm{X}$ curve and have an IQ in the normal range. However, in studies on large groups of fragile X males, Rousseau [Rousseau et al., 1994a] found no males with a full mutation and normal intelligence, and Hagerman [Hagerman R.J. et al., 1994] (subjects 17 and 24 ) reported only $2 / 29$ "high-functioning" fragile X males with a full FMRImutation and complete methylation at the FMRI-associated $\mathrm{CpG}$ island.

Another mechanism to explain the occurrence of "high- functioning" fragile $\mathrm{X}$ males is somatic mosaicism of the mutation. In one tissue, e.g., peripheral lymphocytes, full mutation and premutation, or different lengths of full mutation or premutation, may occur simultaneously. This instability can give either two distinct types of cells in a particular tissue (full mutation / premutation mosaicism), or a whole spectrum of insertion lengths, showing a smear on Southern blot analysis. Fragile X males with a mosaic pattern of CGG insertion 
length in peripheral lymphocytes seem to have a better average IQ than subjects with only full mutation [Hagerman R.J. et al., 1994].

A third proposed possibility is that mosaicism for the length of insertion may not only occur within one tissue, but also between different tissues. Consequently, while in peripheral lymphocytes or another tissue a full mutation is found in a subject, it is possible that the brain cells of this subject carry only the premutation and hence produce FMR1-protein and allow the subject to have a normal IQ. However, at present little is known about the importance of mutation differences between different tissues and hence, whether this mechanism contributes significantly to the occurrence of "high-functioning" fragile X males. For obvious reasons, it is not possible to evaluate the insertion length in brain cells of subjects.

A fourth mechanism has been described recently: in some "high-functioning" fragile X males methylation of the CpG island at the 5' end of the FMRI gene is found in only a fraction of the cells with a full mutation [Hagerman R.J. et al., 1994], [Rousseau et al., 1994b]. The other cells with the full mutation show no methylation at this locus. As methylation represses FMRI-gene transcription [Sutcliffe et al., 1992], one could expect some FMR1-protein to be produced in subjects with a full mutation but partial methylation. Hagerman [Hagerman R.J. et al., 1994] demonstrated FMRI-protein production in two of their "high-functioning" subjects. In these subjects, the amount of FMR1-protein produced was limited, probably because the FMRI mRNA was available only in a limited number of cells. And even in these cells with full mutation and no methylation, a slowdown of FMRI mRNA translation can play a role in limited protein production [Feng et al., 1995].

Combinations of these different mechanisms operating in one subject are also possible, making the molecular-phenotypical relations only more complex. The present study analyses the effect of partial methylation of the $\mathrm{CpG}$-island at the fragile $\mathrm{X}$ site in a group of boys with a full CGG repeat expansion in the FMRI-gene.

\section{Materials and Methods}

\subsection{Subjects}

In our patient population, we found seven boys, between 7-15 years old, with a full mutation at the fragile $\mathrm{X}$ site and an incomplete degree of methylation of the FMRI-gene associated CpG island. The subjects come from five unrelated families: five of them are index patients who presented at the outpatient clinic (subjects $2,4,5$, and 7 ) or were seen in an institution for 
the mentally retarded (subject 6). The two others (subjects 1 and 3) are sibs in two different families. One of the sibs (subject 3) was known to have learning difficulties, and attention and behaviour problems, years before fragile $\mathrm{X}$ was detected in his younger brother (subject 7). In the other pair of sibs, the sib who was not an index subject (subject 1) had not been reported as having problems before detection of fragile $\mathrm{X}$ in his brother (subject 5).

Six of the seven subjects underwent physical examination in our centre. The seventh subject (subject 6) underwent physical examination elsewhere, and his physical phenotype will not be included in the data. Any degree of fragile $\mathrm{X}$ phenotype will be reported as "phenotype present". Intelligence was tested in all subjects with the revised Wechsler Intelligence Scale for Children (WISC-R), Dutch or French version.

Next, we took a behaviour and psychiatric history of all subjects. In five children, the Achenbach's Child Behavior Checklist, Dutch version [Verhulst F. C. et al., 1990], was also taken. We scored as characteristic for fragile $\mathrm{X}$ behaviour phenotype only the combination of attention deficits/ hyperactivity and some form of shyness or social am- bivalence. We also scored "behaviour phenotype present" in two adolescent subjects (subjects 2 and 3) in whom these problems had occurred in earlier phases of development, but were not clinically significant anymore.

\subsection{Molecular Data}

FMRI status was determined on DNA analysis of peripheral lymphocytes. Southern blot analysis using probe St12.3 was performed after double digestion with EcoRI and EagI. When multiple bands of insertion length, or a smear, were found, statistical analysis was performed separately with the minimum and the maximum insertion length. Degree of methylation was determined by densitometry on the Southern blot radiograph. When only a thin streak of methylated DNA and a dense zone of unmethylated DNA were found, limits of precision of densitometry forced us to consider this arbitrarily as methylation in approximately $5 \%$ of cells.

\section{Results}

\subsection{Phenotypic Characteristics (Table I)}

All but one boy of the six who were clinically examined had physical characteristics of fragile $\mathrm{X}$ syndrome. In subjects 2 and 3 this involved only macroorchidism. Subject 4 had craniofacial characteristics and long ears. Subjects 5 and 7 had the full fragile X phenotype. 
Full scale WISC-R (Table I) intelligence quotient (FSIQ) in subjects ranged from 53-119, with an average of 78. Subjects 5-7 go to special education schools. In the sib pairs we found large differences in intelligence level: in the pair subject 1/subject 5 the difference was 63 IQ points or 4 SD (in the WISC-R, 15 IQ points is $1 \mathrm{SD}$ ), and in the pair subject $3 /$ subject 7,30 IQ points or $2 \mathrm{SD}$.

Table 9 Summary of Molecular and Phenotypical Data in seven Boys with Fragile $\mathrm{X}$ mutation.

\begin{tabular}{ccccccc}
\hline $\begin{array}{c}\text { Subject } \\
\text { number }\end{array}$ & Age & $\begin{array}{c}\text { Full-scale } \\
\text { WISC-R IQ }\end{array}$ & $\begin{array}{c}\text { Methylation } \\
(\%)\end{array}$ & $\begin{array}{c}\text { Repeat length } \\
(\mathbf{k b})\end{array}$ & $\begin{array}{c}\text { Phenotypical } \\
\text { features } \\
\text { present }\end{array}$ & $\begin{array}{c}\text { Behavioural } \\
\text { features } \\
\text { present }\end{array}$ \\
\hline 1 & 10 & 119 & 0 & 0.60 & No & No \\
\hline 2 & 13 & 87 & 13 & $0.90($ smear $)$ & Yes & Yes \\
3 & 16 & 83 & 5 & 0.55 & Yes & Yes \\
4 & 7 & 77 & 46 & 0.90 & Yes & Yes \\
5 & 12 & 56 & 88 & 1.10 & Yes & Yes \\
6 & 19 & 70 & 85 & $0.2-2.40$ & Missing data & Missing data \\
\hline 7 & 9 & 53 & 95 & 0.70 & Yes & Yes \\
\hline
\end{tabular}

Subjects I and 5, and subjects 3 and 7, respectively, are two pairs of sibs. In subject 6 only the CpG island associated with the larger CGG insertion $(2.4 \mathrm{~kb})$ was methylated. Methylation refers to percentage of methylation of the FMRI-associated CpG island. Insertion refers to length of expanded CGG repeat in the FMRI-gene.

Five of the six boys who underwent psychiatric examination have or have had behaviour characteristics associated with fragile $\mathrm{X}$ syndrome. Subjects 2 and 3 were known as hyperactive preschoolers and primary school children, though this has disappeared at puberty in both. Subject 3 had been treated for Attention Deficit Hyperactivity Disorder before fragile $\mathrm{X}$ was known in the family. The mutation was found in this family only later, through subject 3 's younger brother, subject 7 . Subjects 2 and 3 have had problems in socializing with peers, with a tendency to withdraw in both. Subject 3 still has these problems. Subject 4 is a hyperactive child and has problems with social ambivalence. Subject 5 is a hyperactive boy. Subject 7 is a shy, hyperactive boy and reacts with tantrums to many external stimuli, especially to acoustic stimuli. Subject 1 also has mild behaviour problems: he has a phobia, not categorized as typical for fragile $\mathrm{X}$ syndrome.

\subsection{Molecular Data}

Four subjects have a homogenous CGG insertion length in the FMRI gene, ranging from 6001,100 base pairs (Figure 3 ). In these subjects, percentage of methylation of the FMRI- 
associated CpG island ranges from $0 \%$ (subject 1) to $95 \%$ (subject 7). Subject 6 has a mosaic mutation with a small CGG repeat insertion around 200 base pairs (* on gel in Figure 3), and a longer smear, with mean insertion length at 2,400 base pairs. In this subject, only the CpG island associated with the longer insertion is methylated. The longer insertion is found in $85 \%$ of lymphocytes. Subject 2 shows a smear centred around 900 base pairs, with $8 \%$ methylation. Subject 3 shows an unmethylated insertion of 550 base pairs and a light streak of methylated DNA, of a corresponding insertion length. This light streak is interpreted as $5 \%$ methylation.

Figure 3 Southern blot assays with probe St12.3 of DNA samples of the seven fragile $\mathrm{X}$ boys

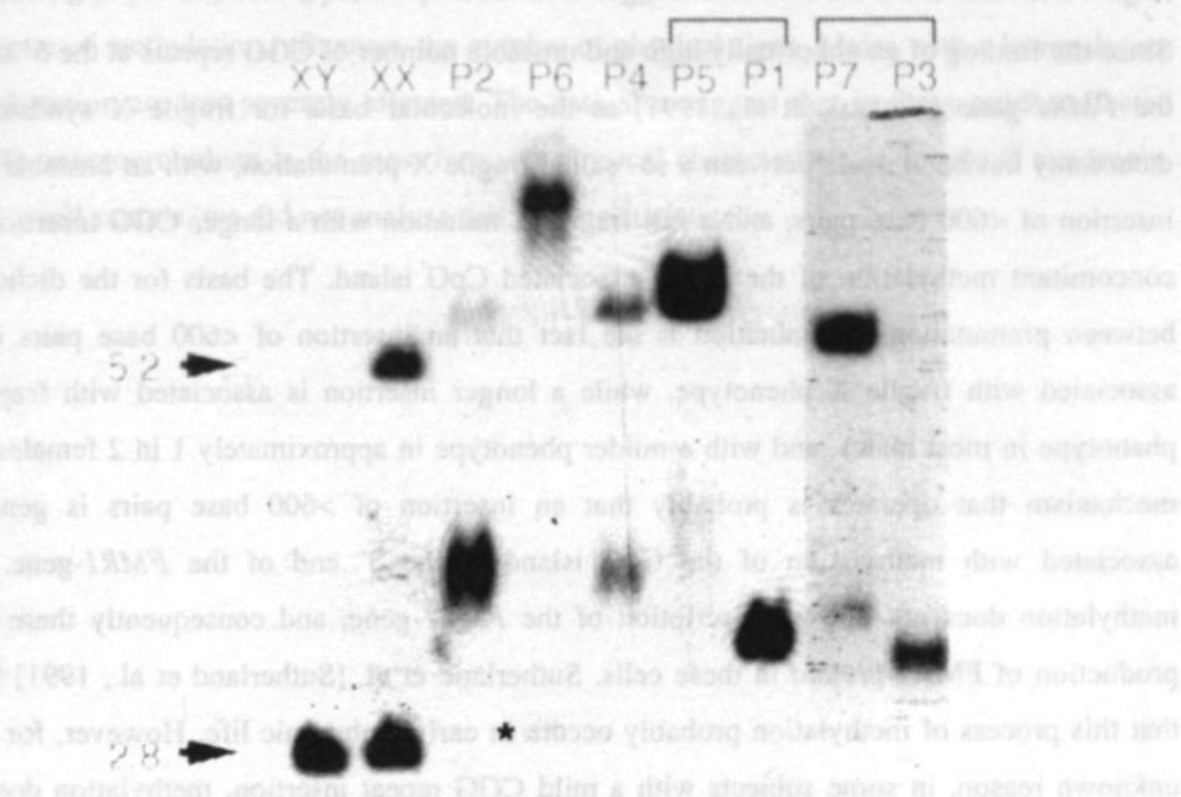

Samples were digested with EcoRI/Eagl. The methylated band at approximately $550 / 600$ hase pairs in subject 3 Mane P3), as seen on the individual gel, cannot be seen in this reproduction; it is interpreted as $5 \%$ methylation.

\subsection{Statistical Analysis}

Pearson correlations between CGG insertion length at the FMRI-gene and degree of methylation of the $F M R I$-associated $\mathrm{CpG}$ island were calculated. There was no significant correlation between insertion length and degree of methylation (Pearson correlation 0.519, F 
$=0.223$ ). In a stepwise regression analysis with IQ as dependent variable, the first step with repeat length as independent variable, could only explain a very small part of variance $(\mathrm{r} 2=$ 0.089 ). When adding degree of methylation to the model, the explained variance significantly increased $(\mathrm{r} 2=0.770)$. This is also reflected in partial regression correlations with IQ (FSIQ) of -0.298 for repeat length and -0.860 for degree of methylation. The multivariate regression analysis is near significance $(\mathrm{F}=0.053)$ in this small sample. Simple regression with degree of methylation as independent variable is significant $(F=0.013)$, but not significant for repeat length as independent variable $(\mathrm{F}=0.516)$.

\section{Discussion}

Since the finding of an abnormally high and unstable number of CGG repeats at the 5' side of the FMRI-gene [Verkerk et al., 1991] as the molecular basis for fragile $\mathrm{X}$ syndrome, a dichotomy has been made between a so- called fragile $\mathrm{X}$ premutation, with an unstable CGG insertion of $<600$ base pairs, and a full fragile X mutation with a longer CGG insertion and concomitant methylation of the FMRI-associated CpG island. The basis for the dichotomy between premutation and mutation is the fact that an insertion of $<600$ base pairs is not associated with fragile $X$ phenotype, while a longer insertion is associated with fragile $X$ phenotype in most males, and with a milder phenotype in approximately 1 in 2 females. The mechanism that operates is probably that an insertion of $>600$ base pairs is generally associated with methylation of the $\mathrm{CpG}$ island at the $5^{\prime}$ end of the FMRI-gene. This methylation does not allow transcription of the FMRI-gene, and consequently there is no production of FMR1-protein in these cells. Sutherland et al. [Sutherland et al., 1991] found that this process of methylation probably occurs in early embryonic life. However, for some unknown reason, in some subjects with a mild CGG repeat insertion, methylation does not occur in all cells. This leads to a phenomenon of partial methylation, in which a fraction of the cells in a particular tissue is methylated at this site and a fraction is not. Previous studies showed that fragile $\mathrm{X}$ males in whom this occurs are less severely affected than males with methylation at- this site in all cells. Hagerman [Hagerman R.J. et al., 1994] demonstrated that this incomplete methylation correlates with some production of FMR1-protein in these males. Thus, absence of methylation in a fraction of cells is one of the mechanisms explaining relatively high functioning in some fragile $\mathrm{X}$ males.

In the present study, incomplete methylation occurred mainly in subjects with a CGG insertion length between 550 and 1,100 base pairs in all lymphocytes. One subject (subject 6) showed mosaicism of $200 / 2,400$ base pairs. We have not yet found subjects with a long 
insertion in all cells and incomplete methylation. One subject (subject 3) shows mild physical and behaviour characteristics of fragile $\mathrm{X}$, while insertion length in peripheral lymphocytes is only 550 base pairs. In this subject, methylation of $\mathrm{CpG}$ is present in a small pro- portion of cells.

The other finding is that in this range of insertion lengths, insertion length per se has no predictive value for intelligence level or penetrance of other phenotypical characteristics. By far the most important predictor of intelligence level is the degree of methylation at the FMRI-associated CpG island. Indeed, linear regression analysis shows that intelligence level correlates negatively with degree of methylation at this site.

Considering physical phenotype, the present data suggest that with the same insertion length, the degree of methylation influences the number of physical signs. Males with a lower degree of methylation are less severely affected. The data also suggest that in these mildly affected subjects, macroorchidism is the most frequent physical characteristic of fragile $\mathrm{X}$ syndrome. In this small sample, we did not analyse this finding statistically. 


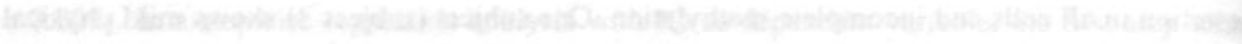

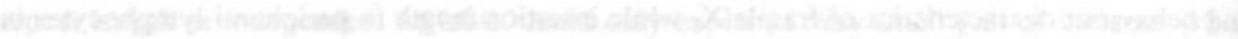

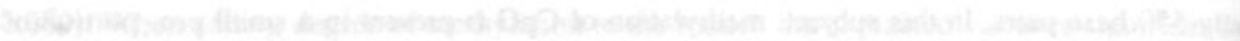

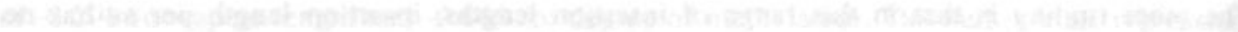

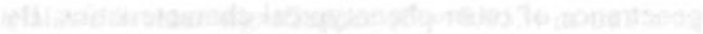

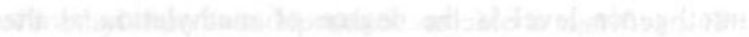

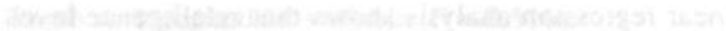

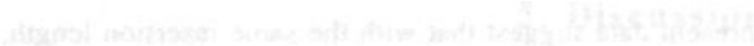

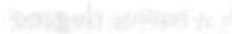

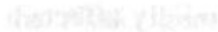

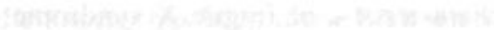




\section{Chapter 8 A distinct neurocognitive phenotype in female fragile $X$ premutation carriers assessed with visual attention tasks.}

J. Steyaert, ${ }^{1,2}$ E. Legius, ${ }^{2}$ M. Borghgraef, ${ }^{2}$ and J.-P.Fryns ${ }^{2}$ (2002)

' Department of Clinical Genetics, University of Maastricht, the Netherlands

${ }^{2}$ Centre for Human Genetics, University of Leuven, Belgium

American Journal of Medical Genetics. In press. 


\section{Abstract}

Premature ovarian failure (POF) and underlying hormonal changes are recognised as a distinct phenotype in female fragile- $X$ premutation carriers. Neurocognitive deficits, in particular mental retardation, are associated with the full mutation in males and females. In female full mutation carriers this neurocognitive phenotype is expressed more mildly than in males. Research on whether the fragile-X premutation is associated with a particular neurocognitive phenotype or not, has been equivocal. By means of the SVAT computer based battery of neurocognitive tasks we assessed reaction time on different tasks in three groups of subjects: female premutation carriers, female full mutation carriers, and female control subjects. The results show that a fraction of the female premutation carriers perform poorly on several selective attention tasks, but not on other tasks. Their neurocognitive profile is different from that of control subjects and of the majority of female premutation carriers. It may also be different from the phenotype of female full mutation carriers, though in that respect this study remains inconclusive. These findings support earlier findings that the fragile-X premutation may affect neurocognitive functioning, in particular aspects of attention. 


\section{Introduction}

Fragile- $\mathrm{X}$ syndrome is the most frequent inherited cause of mental retardation in males [Rousseau et al., 1994a; Rousseau et al., 1995]. The molecular defect consists of an unstable expansion of a CGG trinucleotide repeat within the 5' untranslated region of the FMRI-gene situated at the Xq27.3 fragile-X site of the X chromosome, or FRAXA-site [Verkerk et al., 1991]. Depending on the size of the expansion, the mutation is categorised in two types [Fu et al., 1991; Heitz et al., 1992]: the full mutation, with an expansion of more than 200 repeats ( $>0.6$ kbase), and the premutation, with an expansion between 55 and 200 CGG repeats $(0.15$ 0.6 kbase). The full mutation is associated with the fragile- $\mathrm{X}$ syndrome in males, and less frequently in females [Bardoni et al., 2000; Riddle et al., 1998]. In patients with fragile X syndrome, the expanded CGG triplet repeats in the FMRI promoter region of the full mutation are hypermethylated and the expression of the FMRl-gene is repressed, which leads to the absence of FMR1-protein (FMRP) and subsequent mental retardation [Jin and Warren, 2000]. The premutation is not associated with hypermethylation, and the gene is transcribed to messenger RNA. Recently, it has been demonstrated that premutation carriers have increased levels of FMRI-gene translation [Tassone et al., 2000b] and reduced level of FMRP [Kenneson et al., 2001].

Initially, the premutation was thought not to affect the phenotype of male and female premutation carriers [Cianchetti et al., 1992; Mazzocco and Holden, 1996; Reiss et al., 1993]. However, this view was challenged gradually, as minor anthropomorphic anomalies were described in female permutation carriers [Hull and Hagerman, 1993] and neurocognitive signs in male premutation carriers [Hagerman R.J. et al., 1996] and recently also in a subgroup of premutation carriers [Bennetto et al., 2001]. The most striking and robust finding in female premutation carriers, however, was the occurrence of menopause before the age of 40 due to premature ovarian failure (POF) in approximately one in six female premutation carriers [Allingham-Hawkins et al., 1999; Murray et al., 2000]. Moreover, the prevalence of premutation carriers is significantly increased amongst women with idiopathic POF [Marozzi et al., 2000; Murray et al., 1998; Uzielli et al., 1999]. Remarkably, the prevalence of POF in female full mutation carriers is not increased [Allingham-Hawkins et al., 1999; Murray et al., 2000]. Other reproductive and hormonal anomalies include possible increased twinning in female obligate fragile-X carriers [Fryns J. P., 1986], and specifically in premutation carriers [Vianna-Morgante, 1999], and raised serum FSH in female premutation carriers [Murray et al., 2000]. More recently, anomalies in brain anatomy and metabolism were found in eight 
female premutation carriers [Murphy D.G. et al., 1999], but intelligence does not seem to be affected [Franke et al., 1999].

There is not only growing evidence that female and perhaps male premutation carriers may be different from control subjects. There is also evidence that the pattern of problems is different in premutation carriers compared to full mutation carriers [Murray et al., 2000], and that the premutation carriers do not have a mild expression of the classical fragile $\mathrm{X}$ phenotype, as is the case in subjects with a short full mutation with incomplete methylation [Merenstein et al., 1994; Steyaert et al., 1996]. Female full mutation carriers are characterised by behavioural, neurocognitive and physical features, but not by reproductive anomalies, while reproductive and associated hormonal anomalies are the most robust finding in a fraction of the female premutation carriers. The few neurobiological findings that have been described in female premutation carriers are also different from those in full mutation carriers. While neuroimaging shows increased brain volume of the nucleus caudatus in female and male full mutation carriers [Reiss et al., 1995], the opposite has been described in female premutation carriers [Murphy D.G. et al., 1999]. The neurocognitive findings in female premutation carriers are equivocal: while some researchers [Franke et al., 1999; Mazzocco and Holden, 1996] did not find neurocognitive deficits in female premutation carriers, others [Tassone et al., 2000c] found moderate cognitive deficits in two out of six female premutation carriers. A recent study on neurocognitive functioning in both full mutation and premutation carriers showed problems in executive functions in some premutation carriers [Bennetto et al., 2001]. The available data are insufficient to indicate whether the possible neurocognitive deficits in female premutation carriers are similar to or different from those found in full mutation carriers. Earlier research showed deficits in executive functions in obligate female carriers [Mazzocco et al., 1992] and specifically in female full mutation carriers [Abrams et al., 1994; Sobesky et al., 1994b]. Executive functions include temporal organisation of activities, response selection and control of behaviour. These are controlled processes, which require selective (focused) attention [Franke et al., 1999; Kolb and Whishaw, 1996] also described deficits in sustained attention in female full mutation carriers. The finding of an increased liescale [Sobesky et al., 1994b] or "faking-good profile" [Steyaert et al., 1994] on the Minnesota Multiphasic Personality Inventory in female full mutation carriers might also reflect defective executive functions.

In conclusion, there are arguments supporting the existence of two different phenotypes in female fragile $\mathrm{X}$ carriers. One phenotype is associated with expansions of less than 200 repeats and no hypermethylation at the fragile $\mathrm{X}$ site, and one is associated with more than 
200 repeats and methylation at the fragile $\mathrm{X}$ site. The premutation phenotype is associated with hormonal and reproductive anomalies in females, and possibly with neurobiological and neurocognitive changes, while the full mutation is associated with the classical fragile $\mathbf{X}$ phenotype, or Martin-Bell syndrome. In addition, the different findings raise the question why in the group of female premutation carriers only a minority of subjects are affected. Does this represent the left end of a left-shifted distribution, or a separate group of subjects in the population of premutation carriers?

In order to test this hypothesis further and differentiate possible neurocognitive deficits in female premutation carriers, we assessed a group of female FRAXA carriers with a sensitive computerised test battery that measures different aspects of attention. Objectives are to look whether female premutation carriers, or some of them, have attention deficits, and if so, whether these are different from neurocognitive deficits in full mutation carriers.

\section{Methods}

\subsection{Subjects}

We contacted 40 mothers of fragile-X children and their sisters, known at the Centres for Human Genetics in Leuven and Maastricht. No selection criterion was used. The sisters could be included either as subjects or as controls, depending on what their FRAXA status would prove to be in the study. Thirty-seven of them accepted to participate in the study. Their FMRI repeat status was estimated by Southern blot analysis. Six subjects had an FMRIexpansion in the full mutation range (expansion $>200$ repeats), 27 subjects had an expansion between 55 and 200 repeats, and were classified as premutation subjects. Four sisters had no expansion in the FMRI-region and were classified as normal subjects and put in the control group. Twenty women of non fragile-X families, consisting of students and administrative and technical personnel at the department of genetics, were asked to participate as control subjects. Eighteen of them agreed. FMRI status was not checked in these subjects. Consequently, the subjects were put in either the case group of female FMRI full mutation carriers (6 subjects), the case group of FMRI premutation carriers (27 subjects), or the control group (22 subjects). The age distribution (see Figure 4 ) is significantly different (two sample t-test: $p<0.001$ ) between control subjects and premutation subjects. There is no significant difference $(p=0.478)$ between the control subjects and the full mutation subjects. The difference in age between full mutation and premutation subjects is at the limit of significance 
$(\mathrm{p}=0.052)$. All subjects attended normal school in Belgium or the Netherlands, at least to the age of 16 years. We did not collect additional information on specific learning disabilities at school.

The Raven Progressive Matrices [Raven et al., 1984] was obtained from 16 control subjects and 30 of the 33 subjects with a full or a premutation. This non-verbal intelligence test was taken in order to rule out subjects with a low non-verbal intelligence, as this might influence test performance. All tested subjects scored above the 10th percentile on this preliminary test and none of them were excluded. The three cases and six controls that were not tested had completed at least secondary school and we had no reasons to suspect that they might have an abnormally low intelligence level.

\subsection{Assessment of FRAXA status}

Genomic DNA was collected for Southern blot analysis in the obligate carriers and the control subjects who were family members of fragile $\mathrm{X}$ children, but not in the other control subjects. In 17 premutation cases, the methylation status of the two $\mathrm{X}$ chromosomes was analysed by means of densitometric analysis of the Southern blot electrophoresis gels in order to calculate the activation ratio of the normal X-chromosome [Sobesky et al., 1996].

\subsection{Neurocognitive testing}

The Sonneville Visual Attentions Tasks battery (SVAT) [de Sonneville and Njiokiktjien, 1988] was used to assess aspects of visual information processing in female premutation and full mutation carriers. The computer-based battery is designed to address aspects of visual attention: quality of sustained attention, selective and divided attention. It consists of six different tasks in which reaction times are measured. Each task addresses one or more aspects of visual attention. Conclusions are drawn from the pattern of reaction times in different tasks or subtasks. A particular pattern represents strengths or weaknesses in specific aspects of visual attention. Five essential tasks of the battery were taken from all subjects. Different tasks have more than one measured variable, e.g. reaction time for correct responses and reaction time for incorrect responses. In this study, the reaction times for correct responses were used. The error rates are also reported.

The first task, the Finger Motor Speed Exercise (FMSE), is a baseline test of reaction speed under the lowest cognitive demands possible. It is used to assess whether pure motor slowness may have a significant influence on performance on the other tasks.

The Letter Pattern Exercise (LPE) addresses aspects of focussed as well as of divided attention. Subjects have to remember one or more target letters, and press a "correct" button 
when the letter(s) appear(s) in consecutive square frames containing four letters. When the target letter is not present, the subjects actively respond by pressing a "false" button. In each frame, the subjects are confronted with a new set of four letters, that may or may not contain the target letter. As each frame can be different from all the previous ones, automatic processing of the task is not possible. The subjects have to focus intensely on each new set of four letters.

The Focussed Attention Exercise (FAE) also requires selective attention and response selection. It resembles the LPE, with the additional difficulty that the subjects may only give a "correct" response when the target letter is presented on a particular diagonal.

The Dot Pattern Exercise (DPE) is a rather dull task with low cognitive load that addresses sustained attention. Demands on selective attention are much lower in this task. The subjects are presented 600 consecutive frames with three, four or five dots. They give a "correct" response (hit) when four dots appear, and a "false" response (correct rejection) when three or five dots appear. Subjects seem to automatise this task easily, as there are only three possibilities, and after less then ten of the 600 frames, the subjects have seen the three possibilities. To limit the total of variables to analyse, we selected only the reaction time for the hits (four dots).

In the Compatible/Incompatible Response Exercise (CIRE) the subjects are shown 60 consecutive frames with the words "right" or "left", printed either in white letters on a black screen, or in black letters in a white background frame. When the letters are in white, the left or right-hand button should be pressed, compatibly with the word left or right. When the letters are in black, the reverse response should be given. In the SVAT, this is called an incompatible response. As there are only four possible responses, a subject has seen them all soon, and processing is at least partly automatic. Even if the level of attention has to remain high. In the statistical analysis, reaction times for left and right compatible responses were clustered (TCOMP), as well as the reaction times for left and right incompatible response (TINCOMP).

\subsection{Data analysis}

All statistical tests were performed using the Systat 7.0 package [SYSTAT®, 1997]. Two control subjects, one premutation subject and one full mutation subject were left-handed. The effect of handedness on task performance is assessed by t-tests comparing the reaction times of left-handers with the group mean for right-handers of each category of subjects. 
Stepwise discriminant analysis was performed to analyse which of the SVAT variables is the strongest discriminator between the three groups of subjects, with the rationale that if there is a latent fourth group (unusual premutation subjects), analysing the distribution of the most differentiating variable would be the best approach to separate this fourth group.

As some groups contained less than 20 subjects, we used non-parametric two-sample Kolmogorov-Smirnov test to assess between group differences. Non-parametric tests are more rigorous while parametric tests may yield falsely positive significance in small groups. This does not allow analysis of covariance however. Classical parametric analysis of covariance (ANCOVA) was used to analyse the effects of intelligence and age as co-variates.

\section{Results}

\subsection{Effect of handedness}

T-tests comparing left-handed subjects with right-handed subjects were performed for all the reported SVAT-variables, but did not show influence of handedness. The results of these ttests are not reported further here.

\subsection{Discriminant analysis}

Stepwise discriminant analysis aimed at detecting which of the SVAT tasks could differentiate best between the three groups of subjects, yielded a satisfactory prediction model ( $\mathrm{p}=0.051$ ) including the variables. In this model the reaction time for correct responses on the LPE has the highest F-value, $F=2.63$, and was therefore selected as discriminating variable. The task LPE correlates moderately to highly with other SVAT tasks, and has low correlations with the independent variables age (Pearson correlation 0.215 ) and non-verbal intelligence as assessed with the Raven Progressive Matrices intelligence test (Pearson correlation -0.342). The good differentiating power and the low correlation with age and intelligence make LPE a suitable variable to analyse further whether subgroups can be found in within the group of female premutation carriers.

\subsection{Premutation carriers versus controls}

As two-sample Kolmogorov-Smirnov (KS) analysis showed significant group differences between premutation carriers and controls for the variables age (KS: $p=0.001)$ and Raven IQ (KS: $p=0.007$ ) (see Table I), we performed analysis of covariance (ANCOVA) on LPE with the covariates age and Raven IQ. ANCOVA for LPE with age as covariate did not yield significant differences between the premutation carriers and controls: $p=0.147$. ANCOVA for LPE with Raven IQ as covariate yielded similar results: $\mathrm{p}=0.278$. 


\subsection{Selection of "slow" premutation subjects:}

In the next step we evaluated the distribution of this variable in the group of premutation carriers, which proved to be markedly broader then for the normal controls (see Figure 4): standard deviation of correct reaction time on the LPE task in normal controls is $67 \mathrm{msec}$ (mean $588 \mathrm{msec}$ ), while it is $139 \mathrm{msec}$ in premutation carriers (mean $650 \mathrm{msec}$ ). Subsequently we selected as "slow" premutations the premutation carriers with scores on LPE exceeding two standard deviations above the mean of the normal controls. This yielded a group of five premutation carriers, with markedly slower reaction times on LPE, with a range between 741 and $1122 \mathrm{msec}$. (Fig. 1.) The remaining premutation carriers are defined as "regular" premutations.

\subsection{Characteristics of the "slow premutation" subjects}

Two-sample KS analysis between controls and the group of regular and slow premutations respectively, yielded no significant group differences for Reaction Time $(\mathrm{T})$ without cognitive load. There were however significant differences between controls and slow premutation subjects on the LPE task while performances of the regular premutation subjects where not different from that of the controls on these tasks. Error rate on the LPE was not significantly different between the three groups. Performance of the three groups on the FAE task showed a similar pattern as the LPE: significant differences on all tasks between the control subjects and the slow premutation subjects, as well as between the regular and slow premutation subjects, but no differences between the control and regular premutation subjects. Error rates on the FAE tasks are not different for the three groups. There were no significant differences between controls and slow premutations on the DPE task for sustained attention. On the task Compatible Incompatible Response Exercise (CIRE) there were different performances between the controls and slow premutation subjects, which were significant for the compatible (TCOMP) but not for the incompatible (TINCOMP) responses. The difference between regular and slow premutation subjects was more pronounced and reached significance for both variables. Error rates on all the tasks are not significantly different between the three groups 
Table 10 Molecular data, mean reaction times and error rates of the normal controls, premutation subjects, and full mutation subjects for the selected SVAT tasks.

\begin{tabular}{|c|c|c|c|c|c|c|c|c|c|}
\hline & \multirow{2}{*}{$\begin{array}{c}\begin{array}{c}\text { Normal } \\
\text { controls }\end{array} \\
(n=22)\end{array}$} & \multicolumn{2}{|c|}{ All Premutations } & \multicolumn{2}{|c|}{$\begin{array}{c}\text { Regular } \\
\text { Bremutations }\end{array}$} & \multicolumn{3}{|c|}{$\begin{array}{c}\text { Slow } \\
\text { premutations }\end{array}$} & \multirow{2}{*}{$\begin{array}{c}\text { Mutations } \\
(n=6)\end{array}$} \\
\hline & & $(n=27)$ & $\begin{array}{c}\text { KS-test } \\
\text { Controls } \\
\text { versus } \\
\text { premutations } \\
\end{array}$ & $(n=2.2)$ & $\begin{array}{c}\text { KS } \\
\text { Controls } \\
\text { versus regular } \\
\text { premutations }\end{array}$ & $(n=5)$ & $\begin{array}{c}\text { KS } \\
\text { Controls } \\
\text { versus slow } \\
\text { premutations }\end{array}$ & $\begin{array}{c}\text { KS } \\
\text { Regular } \\
\text { versus slow } \\
\text { premutations }\end{array}$ & \\
\hline Age (years (sd)) & $27.3(10.9)$ & $38.9(9.2)$ & $p=0.001$ & $39.2(8.7)$ & $p=0.001$ & $37.8(12.1)$ & $p=0.027^{*}$ & $p=0.807$ & $30 . r(8.2)$ \\
\hline Repeat length (kb (sd)) & n.a. & $0.29(0.11)$ & & $0.30(0.12)$ & & $0.26(0.09)$ & & $p=0.548$ & $1.72(0.46)$ \\
\hline$\% \mathbf{X}$-activation ( $\%$ (sd)) & n.a. & $62.4(28.2)$ & & $72.7(2+.7)$ & & $28.7(19.6)$ & & $\mathrm{p}=0.048^{*}$ & \\
\hline Raven IQ raw scores (sd) & $10.1(1.8)$ & $8.0(1.8)$ & $p=0.007$ & $8.1(1.9)$ & $p=0.005$ & $7.8(1.8)$ & $p=0.906$ & $p=0.929$ & $7.5(0.7)$ \\
\hline \multicolumn{10}{|l|}{ SVAT $^{*}($ times in msex (sd)) } \\
\hline Reaction time (T) & $278(50)$ & $285(49)$ & $p=0.668$ & $284(56)$ & $p=0.799$ & $289(42)$ & $p=0.507$ & $p=0.927$ & $299(42)$ \\
\hline \multicolumn{10}{|l|}{ Letter Pattern Exercise (LPE) } \\
\hline Correct responses & $588(67)$ & $650(139)$ & $p=0.108$ & $598(6(6)$ & $p=0.547$ & $876(156)$ & $p<0.001 *$ & $p<0.001 * *$ & $691(98)$ \\
\hline Error rate (sd) & $1.8 \%(3.2)$ & $1.4 \%(2.7)$ & $\mathrm{p}=0.968$ & $1.6 \%(2.8)$ & $p=1$ & $1.0 \%(2.2)$ & $p=0.098$ & $p=0.098$ & $1.6 \%(2.5)$ \\
\hline \multicolumn{10}{|l|}{ Dot Pattern Exercise (DPE) } \\
\hline Correct responses & $673(104)$ & $676(112)$ & $p=0.549$ & $684(7.3)$ & $p=0.799$ & 799 (176) & $p=0.164$ & $p=0.063$ & $774(153)$ \\
\hline average error rate (sd) & $1.0 \%(0.6)$ & $0.7 \%(0.4)$ & $p=0.353$ & $0.7 \%(0.4)$ & $p=0.325$ & $0.6 \%(0.6)$ & $\mathrm{p}=0.487$ & $p=0.761$ & $0.8 \%(0.4)$ \\
\hline \multicolumn{10}{|c|}{ Focused Attention Exercise (FAE) } \\
\hline Correct responses & $550(83)$ & $571(120)$ & $\mathrm{p}=0.539$ & $529(87)$ & $p=0.216$ & $756(42)$ & $p<0.001 \cdots$ & $\mathrm{p}<0.001 *$ & $638(80)$ \\
\hline Error rate (sd) & $5.6 \%(6.6)$ & $4.4 \%(4.6)$ & $\mathrm{p}=0.923$ & $4.1 \%(5.0)$ & $p=0.970$ & $6.0 \%(2.2)$ & $p=0.457$ & $p=0.321$ & $\begin{array}{c}10.8 \% \\
(7.3)\end{array}$ \\
\hline \multicolumn{10}{|l|}{ Compatible/incompatible } \\
\hline Compatible responses (TCOMP) & $569(123)$ & $593(142)$ & $p=0.747$ & $563(10,5)$ & $p=0.799$ & $729(215)$ & $\mathrm{p}=0.050^{*}$ & $\mathrm{p}=0.027^{*}$ & $642(109)$ \\
\hline Incompatible responses(TINCOMP) & $596(199)$ & $595(162)$ & $\mathrm{p}=0.648$ & $563(129)$ & $\mathrm{p}=0.798$ & $739(228)$ & $p=0.209$ & $\mathrm{p}=0.050^{*}$ & $614(97)$ \\
\hline average error rate (sd) & $2.9(3.3)$ & $2.6(1.9)$ & $\mathrm{p}=0.853$ & $3.3(2.2)$ & $\mathrm{p}=0.547$ & $1.2(1.4)$ & $\mathrm{p}=0.703$ & $\mathrm{p}=0.155$ & $3.3(2.2)$ \\
\hline
\end{tabular}

- All reaction time data represent means and standard deviation (sd) for the givein group. KS = probability assessed with the two-sample Kolmogorov-

Smirnov test. Levels of statistical significance: ${ }^{*}: p<0.05 ; * * ;<0.01$. 
Two-sample KS-analysis on the variable age showed significant differences between controls and regular premutations, as well as between controls and slow premutations. There was no significant difference between the slow and the regular premutation subjects. Pearson correlations between age and the different SVAT tasks were not significant (not shown). As KS analysis had shown a significant group difference between slow premutations and controls for the variable age, we performed ANCOVA for LPE with age as covariate. The group effect controls versus slow premutation remained significant: $\mathrm{p}<0.001$.

Figure 4 Distribution of reaction time in milliseconds for LPE for the four groups of subjects.
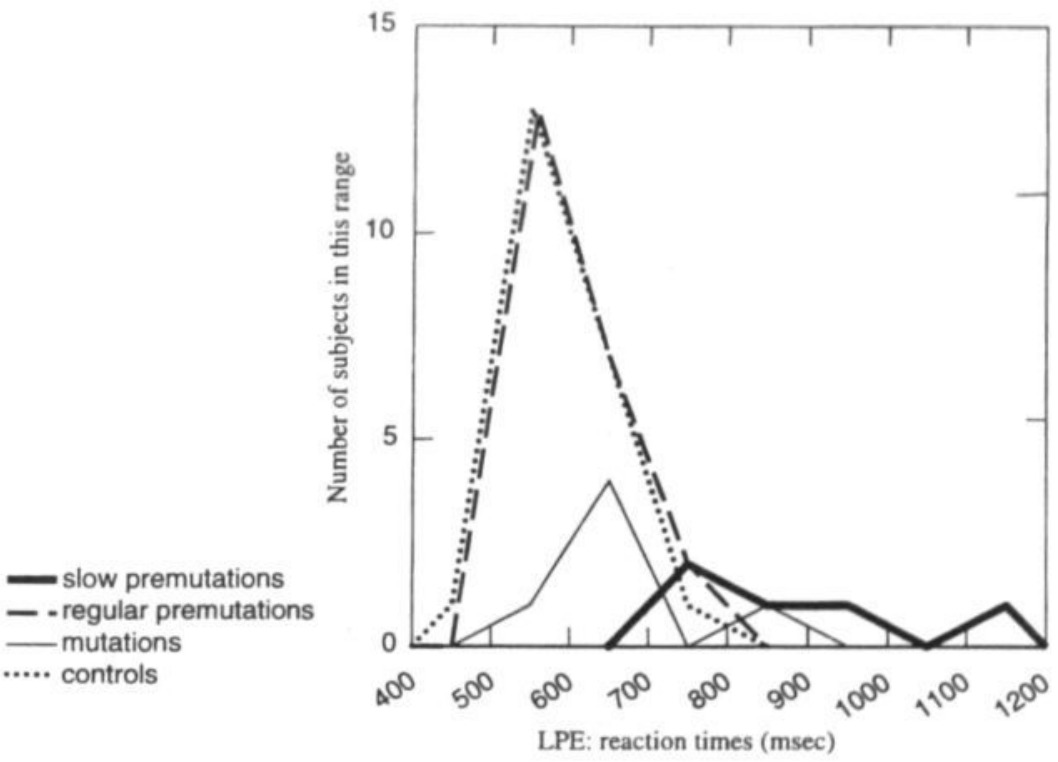

\subsection{Molecular data}

Two-sample KS-analysis for premutation length did not show significant differences ( $\mathrm{p}=$ 0.548 ) between regular premutation (mean expansion length $0.30 \mathrm{~kb} ; \mathrm{sd}=0.12$ ), and slow premutation subjects (mean expansion length $0.26 \mathrm{~kb}$; $\mathrm{sd}=0.09$ ). We obtained $\mathrm{X}$-activation ratio for the normal FMRI-gene for 13 of the 22 regular premutation subjects and for 4 of the 5 slow premutation subjects. Mean X-activation ratio in the whole group of 27 premutation 
carriers was $62.4 \%(\mathrm{sd}=28.2, \mathrm{Cl}=47.9 \%$ to $76.9 \%)$, which is a non-significant difference $(\mathrm{p}=0.09)$ from the expected $50 \%$. Between groups $\mathrm{KS}$-analysis for differences in $\mathrm{X}$-activation ratio showed a significant difference $(\mathrm{p}=0.048$ ) between the regular premutation group (mean $\mathrm{X}$-activation ratio $72.7 \%$, sd=21.7) and the slow premutation group (mean $\mathrm{X}$-activation ratio: $28.7 \%, s d=19.6$ ).

\section{Discussion}

We assessed different aspects of attention in 27 female FRAXA premutation carriers, 6 female FRAXA full mutation carriers, and 22 control subjects with the de Sonneville Visual Attention Tasks battery, in order to see whether these specific neurocognitive tasks could shed evidence on the existence of a separate phenotype in female FRAXA premutation carriers. The mean $\mathrm{X}$-activation ratio of the group of 27 premutation carriers was non-significantly skewed $(62.4 \%)$. We have no other explanation than that this is a chance effect in a relatively small group. Though the group of premutation carriers where significantly older than the control subjects and had a lower Raven IQ, analysis of covariance did not show any significant effects of these variables on the measured reaction times. Obviously, the differences in age and intelligence where not that large that they could account for differences in task performance. There were no significant differences on any SVAT task between the control subjects and the total group of premutation carriers.

By means of statistical analysis, within the total group of 27 female premutation carriers we differentiated a group of five who performed significantly different from control subjects and from other premutation carriers. These $5(18.5 \%)$ premutation carriers are characterised by slower information processing speed on tasks with a high demand on controlled processing (selective attention and complex response selection), but not on tasks that require mainly automatic processing and/or sustained attention. The 22 other premutation carriers do not differ from normal controls on any of the attention tasks. In our sample, these "slow" premutation carriers are not different from the other premutation carriers in terms of intelligence, age, nor repeat length. However, both groups differ significantly in X-activation ratio in peripheral lymphocytes: the X-activation ratio is $72.7 \%$ in the "regular" premutation carriers, and $27.8 \%$ in the "slow" premutation carriers. Lower expression of FMR protein and higher expression of FMRI-messenger RNA (mRNA) have been observed in some premutation carriers [Kenneson et al., 2001; Tassone et al., 2000c]. It is not clear how this may affect neurological development and neurocognitive functioning. Lower $\mathbf{X}$-activation ratios might be associated with lower FMRP expression and/or higher FMRI-mRNA levels. 
In the present research, we did not have the opportunity to test the premutation carriers for FMRP expression and FMRI-mRNA levels. This has to be investigated further.

The fact that tasks requiring a more difficult response selection and attentive processing are impaired in the "slow" premutation carriers, while sustained attention is not, suggests that frontal lobe functions may be more impaired in these subjects. Indeed, response selection is highly mediated by frontal lobes [Naätänen and Sokolov, 1992], as are other executive functions like temporal organisation [Kolb and Whishaw, 1996]. Some researchers who also assessed executive functions in female premutation carriers [Franke et al., 1999; Mazzocco and Holden, 1996; Thompson et al., 1994] found no differences with normal controls. Our findings rather confirm the recent finding of problems in executive functioning in a subgroup of premutation carriers [Bennetto et al., 2001]. The different findings may be caused by the fact that in earlier studies the premutation carriers were analysed as a homogeneous group. We purposefully tried to detect a different subgroup, in following of the findings with POF in premutation carriers, where POF also seems to affect only a subgroup of female premutation carriers. Another reason for these different findings may be the fact that in this study we used a sensitive test to measure aspects of attention, of which focused attention is one. Earlier studies focused more on executive functions related to temporal organisation and flexibility [Franke et al., 1999; Mazzocco and Holden, 1996; Thompson et al., 1994].

Comparison of the "slow" premutation carriers with full mutation carriers shows that the "slow" premutation carriers are more impaired than the full mutation carriers on most tasks, and that the difference is the largest in the tasks requiring selective attention. However, not all the differences between the two groups are significant, possibly because both groups are too small in this study. The comparison of these two groups is more equivocal: the impairment of executive functions appears to be more important in "slow" premutation carriers than in full mutation carriers, but the profile of impairment (selective attention more impaired than sustained attention) is rather similar. Consequently, though it is clear that a subgroup of female premutation carriers have a different neurocognitive phenotype than the majority of premutation carriers, who are similar to control subjects, it is inconclusive whether these "slow" premutation carriers have another neurocognitive phenotype than the full mutation carriers. However, it is unlikely that these $18.5 \%$ "slow" premutation carriers are all women with a tissue mosaicism for the FRAXA full mutation, with full mutation in a substantial part of brain cells, and premutation in the peripheral lymphocytes: the unstable expansion seems to acquire mitotic stability in very early foetal life [Wohrle et al., 2001], and while expansion mosaicism is common within tissues [Wohrle et al., 2001], large expansion differences 
between different tissues are not a frequent phenomenon in fragile $\mathrm{X}$ [Allingham-Hawkins et al., 1996; Reyniers et al., 1999; Tassone et al., 1999a; Wohrle et al., 2001].

This study cannot show whether the premutation carriers with this different neurocognitive phenotype are the same as the ones with premature ovarian failure (POF), as most subjects are younger than the age at which POF occurs. Assessment of their hormonal status may give additional evidence on this issue. A final limitation is that we have insufficient data on the school career of the subjects to discriminate whether the "slow" premutations had more learning problems reflecting weakness in executive functions -e.g. ADHD-like symptomsthan the other premutations or control subjects. 


\section{Chapter 9 A study of the cognitive and}

\section{psychological profile in 16 children with congenital or juvenile myotonic dystrophy}

Jean Steyaert', Stijn Umans ${ }^{2,3}$, Diane Willekens ${ }^{2}$, Eric Legius ${ }^{2}$, Elly Pijkels $^{2}$, Christine de Die-Smulders ${ }^{1}$, Herman Van den Berghe ${ }^{2}$ and Jean-Pierre Fryns ${ }^{2}$ (1997)

'Centre for Clinical Genetics, University of Maastricht, the Netherlands.

${ }^{2}$ Centre for Human Genetics, University of Leuven, Belgium.

${ }^{3}$ Department of Child Psychiatry, University of Leuven, Belgium.

Clinical Genetics; 52(3): 135-41. 


\section{Abstract}

We report data on intelligence and on possibly associated psychopathology in 16 children and adolescents, between 7 and 18 years of age with congenital or juvenile myotonic dystrophy (DM). We found that all the subjects have an intelligence level below the population mean, four of them in the mentally retarded range. An unexpected number of subjects are in the clinical range on the Child Behaviour Checklist. In nine of the sixteen subjects, a child psychiatric diagnosis was found using a standardised psychiatric interview. The most frequent child psychiatric diagnosis was Attention Deficit Hyperactivity Disorder. 


\section{Introduction}

Myotonic Dystrophy (DM) is a progressive neuromuscular disease characterized by a wide variation in neuromuscular symptoms and multisystem involvement. Besides myotonia and progressive muscular weakness other symptoms can be present, depending on the affected organ system: cataract, smooth muscle disturbances (esophageal spasms, spastic colon, diarrhoea), gonadal dysfunction (subfertility), mental retardation, depression, somnolence, diabetes, and cardiac conduction defects (bradycardia, arrythmias) [Harper, 2001].

D.M. is an autosomal dominant inherited disorder, caused by an expanded and unstable CTG repeat sequence in the $3^{\circ}$ untranslated region of a gene on chromosome 19 . The sequence of this gene predicts its protein product to be a member of the protein kinase family [Aslanidis et al., 1992; Brook et al., 1992; Buxton et al., 1992; Harley et al., 1992a; Harley et al., 1992b; Shaw et al., 1993].

The prevalence of the DM-mutation in the general population is estimated to be 1 in 8000 [Hunter et al., 1992].

Harley proposed a classification of four different categories of myotonic dystrophy in relation to age of onset and of clinical symptoms [Harley et al., 1993].

1. The mild form of DM with cataract and minimal or no neuromuscular symptoms in middle or older age.

2. The classical or adult form with typical neuromuscular symptoms in adolescence or early adult life.

3. The juvenile or childhood form: symptoms are present in childhood before the age of 12 years. Learning disabilities are often prominent, while neuromuscular symptoms are rather mild or sometimes even absent.

4. The congenital form with clinical symptoms present from birth on or in utero: hypotonic cerebral palsy, respiratory and/or feeding problems, and mild to moderate developmental delay in survivors.

The psychiatric and cognitive evolution of individuals with the adult form of DM has been well documented in the past, with controversial findings. Studies rather suggest an increased incidence of depression in the group of patients with DM [Cuthill et al., 1988; Harper, 2001]. It is equivocal whether the presence of a carefree temperament is typical for patients with DM [Harper, 2001]. Some studies in adults suggest intellectual impairment and lower IQ as age of onset of signs and symptoms is lower, but this is not confirmed in other studies [Broughton et al., 1990; Censori et al., 1990; Perini et al., 1989; Ragazzoni et al., 1991; Ragazzoni et al., 
1993; Tuikka et al., 1993; Turnpenny et al., 1994; Van Spaendonck et al., 1995]. Apart from the fact that neuromuscular symptoms are frequently associated with developmental problems, not much is known about the cognitive and behavioural aspects of the infantile and juvenile form of DM.

We studied intelligence and possibiy associated psychopathology in 16 children and adolescents, between 7 and 18 years of age, with either the congenital ( 2 patients), or the juvenile form (14 patients) of DM. The study is also the first phase of a longitudinal study on possible cognitive and developmental changes in subjects with infantile or juvenile DM.

\section{Subjects and methods}

\subsection{Subjects}

We studied a group of 16 children and adolescents, between the age of 7 and 18 years, 9 girls and 7 boys. Eleven were index-patients in their respective families and all were referred to the centre for clinical genetics because of a great variety of neuromuscular problems (hypotonia, somnolence, ...etc.), other somatic problems (gastrointestinal problems), or developmental problems and learning difficulties. The other five subjects are affected brothers and sisters of the index patients. The clinical diagnosis of DM was confirmed by molecular studies and further family studies were performed. Table 1 gives a summary of the age of onset of clinical symptoms, the present age and the molecular data in the 16 children and adolescents and of the molecular data in the affected parents. All affected parents presented a classic, adult onset DM.

History and clinical findings allowed classifying the 16 subjects in two groups, following the classification we propose:

\section{a) Congenital DM group (CDM) (2 girls)}

Severe hypotonia was noted at birth in the two children. A foetal hypokinesia sequence with reduced foetal movements and polyhydramnios was seen in one child. One child presented at birth a talipes equinovarus deformity of both feet; one other a respiratory distress syndrome, and both children had serious feeding- and swallowing difficulties from the beginning. They had facial weakness with typical tented upper lip, myotonia, dysarthric speech and extreme slow movements. One is mildly mentally retarded (FSIQ between 50 and 70) and one has a borderline intelligence (FSIQ between 70 and 85 ). 


\section{b) Juvenile DM group (JDM) (14 patients; 7 boys and 7 girls)}

No major problems were noted in these children at birth. Five of them had feeding problems (slow suckers; bottle-feeding was started after birth because breast feeding was not possible), while pre- and perinatal history was normal in seven patients. The five patients with early feeding problems were diagnosed as having DM before the age of eight, based on the following symptoms: muscular hypotonia (1 child), tiredness after physical exercise ( 1 child), gastrointestinal symptoms (1 girl with feeding difficulties with constipation and diarrhoea), learning difficulties (all five). The others were diagnosed before the age of 15 years based on the following: learning difficulties ( 4 patients), muscular hypotonia ( 2 patients), and somnolence ( 1 patient).

Data on intelligence were as follows: mild mental retardation ( 3 subjects), borderline intelligence ( 5 subjects), learning difficulties with IQ's between 85 - 100 ( 5 subjects). Nine of the 12 children are in a special education program. The fourteen children with JDM were hypotonic and presented facial weakness, and myotonia in the face and the hands. Delayed fine motor development was noted in five of them and somnolence in four others.

Most children in both groups needed a rather long night rest: 12 of the 16 children needed 10 or more hours of sleep a day.

\subsection{Cognitive testing}

Intelligence was tested with the Wechsler scales: WISC-R scale in the children (Van Haasen et al. 1974) and WAIS scale (Stinissen et al. 1970) in the adolescents older than 16 years. These scales are subdivided in a performal and verbal subscale. Each subscale contains different subtests (verbal subscale: information, similarities, arithmetic, vocabulary, comprehension and digit span; performal subscale: picture completion, picture arrangement, block design, object assembly, substitution and mazes). The resulting data give us a total intelligence quotient (FSIQ), a verbal intelligence quotient (VIQ), a performal intelligence quotient (PIQ) and an intelligence profile. Differences over time in FSIQ in a single patient are present when the $95 \%$-reliability intervals of the first and the last IQ result do not overlap [Van Haasen et al., 1974]. Thus, a decline in IQ can be demonstrated with a probability below 0.05 .

\subsection{Behavioural measures}

The Dutch version of the Children Behavior Checklist (CBCL) [Verhulst F. C. et al., 1990], a well-validated checklist standardized in children with normal and borderline intelligence (IQ $>70$ ), was completed by the parents. The results from this checklist are transformed in a total 
problem score, in two subscores for respectively the internalising (withdrawn, somatic complaints and anxious/depressed) and externalising problem behaviour (delinquent and aggressive) and in a profile of clusters with specific problem behaviour (withdrawn, somatic complaints, anxious/depressed, social problems, thought problems, attention problems, delinquent behaviour and aggressive behaviour). The responses are transformed into T-scores $(X=50$, s.d. $=10)$. In the total problem score, and the internalising and externalising subscores, a T-score of 63 or higher (coinciding with 90 th centile or higher) are considered to be of concern and are called by the authors as being "in the clinical range". T-scores in the range of 70 or higher (> 98th centile) are found in populations with severe behaviour or emotional problems. For the specific problem clusters T-score of 66 or higher (>93rd centile) are considered to be of concern [Verhulst F. C. et al., 1990].

Depressivity was assessed by two questionnaires, adapted for a Dutch population: the Child Depression Scale [Luteijn, 1981] in 9 children younger than 15 years, and the Beck Depression Inventory [Beck et al., 1961; Bouman et al., 1985] in 5 adolescents older than 15 years.

Finally, the possible presence of clinical child psychiatric syndromes was assessed by means of a structured parent-interview: the Dutch version of the Diagnostic Interview for Children and Adolescents (ADIKA) (Kortenbout et al. 1993). This instrument, based on the criteria of the DSM-III-R classification, has been adapted in Dutch, but the norm values for a Dutch population are not available yet. Therefore, we applied the DSM-III-R criteria [American Psychiatric Association, 1987] and the U.S.-norms of the Diagnostic Interview for Children and Adolescents [Reich et al., 1982; Welner et al., 1987].

The length of the insertion at the DM-site was assessed on peripheral lymphocytes. Genomic DNA was extracted and two Southern blots were performed: one to assess longer insertions, after digestion with EcoRI, and one to assess shorter insertions, after digestion with BgII. Standard techniques were used, with p25B1.4 as a probe. Repeat lengths were measured and expressed as difference in repeat size with a normal control.

\section{Results (Table 11)}

\subsection{Molecular data}

The mean repeat size was $3.55 \mathrm{~kb}$ in the two subjects with congenital $\mathrm{DM}, 1.88 \mathrm{~kb}$ in the group of juvenile DM. All affected parents presented a classical adult form of DM. The mean repeat size in the transmitting mothers was $0.66 \mathrm{~kb}$ and $2.3 \mathrm{~kb}$ in their affected children. The 
mean repeat size was $1.2 \mathrm{~kb}$ in the transmitting fathers and $1.5 \mathrm{~kb}$ in their affected children. ANOVA of repeat size between the two categories shows significant differences between the categories ( $\mathrm{p}=0.001$, multiple $\mathrm{r} 2=0.61$ ).

\subsection{Intelligence}

The full scale IQ (FSIQ) was below I00 in all 16 subjects; individual IQ's varied between 62 and 95, and the mean FSIQ was 79. Average FSIQ (Table 2) in the category Congenital DM (CDM) was 68, and 80 in the category juvenile DM (JDM).

ANCOVA of FSIQ between the two categories of DM, with age as co-variate, shows significant differences $(p=0.039)$ between these categories. Regression analysis of FSIQ in function of in the whole group of subjects shows an inverse correlation $(p=0.025)$.

Table 11 Type of DM, sex, age, insertion length, transmitting parent data, full scale Wechsler IQ and psychiatric diagnosis in 16 DM subjects.

\begin{tabular}{|c|c|c|c|c|c|c|c|c|}
\hline & Sex & $\begin{array}{l}\text { Present } \\
\text { age }\end{array}$ & $\begin{array}{l}\text { Age at } \\
\text { diagnosis }\end{array}$ & $\begin{array}{c}\text { CTG } \\
\text { repeat } \\
\text { size (kB) }\end{array}$ & $\begin{array}{c}\text { Full } \\
\text { scale IQ }\end{array}$ & $\begin{array}{l}\text { Child psychiatric } \\
\text { diagnosis }\end{array}$ & $\begin{array}{c}\text { Affected } \\
\text { parent }\end{array}$ & $\begin{array}{c}\text { CTG } \\
\text { repeat } \\
\text { size in } \\
\text { parent }\end{array}$ \\
\hline Congenital & female & 16 & 3 & 3.6 & 73 & earlier ADHD & mother & 1 \\
\hline $\begin{array}{l}\text { Myotonic } \\
\text { Dystrophy }\end{array}$ & female & 18 & 7 & 3.5 & 62 & $\begin{array}{c}\text { separation anxiety } \\
\text { disorder }\end{array}$ & mother & 0.5 \\
\hline \multirow{14}{*}{$\begin{array}{l}\text { Juvenile } \\
\text { Myotonic } \\
\text { Dystropy }\end{array}$} & female & 7 & 7 & 1.6 & 78 & $\begin{array}{c}\text { adjustment } \\
\text { disorder }\end{array}$ & mother & unknown \\
\hline & male & 9 & 7 & 2.2 & 71 & ADHD & mother & 0.35 \\
\hline & female & 10 & 8 & 2.1 & 65 & $\begin{array}{c}\text { generalized anxiety } \\
\text { disorder }\end{array}$ & mother & 0.35 \\
\hline & male & 11 & 8 & 1.2 & 83 & ADHD & father & 0.7 \\
\hline & female & 17 & 3 & 1.5 & 90 & none & mother & 1.4 \\
\hline & female & 11 & 9 & 2.6 & 87 & none & mother & 0.3 \\
\hline & female & 12 & 3 & 1.9 & 74 & $\begin{array}{c}\text { separation anxiety } \\
\text { disorder }\end{array}$ & father & 2.4 \\
\hline & male & 12 & 4 & 1.0 & 89 & none & father & 0.5 \\
\hline & female & 14 & 4 & 2.0 & 65 & none & mother & 0.5 \\
\hline & male & 14 & 11 & 1.5 & 95 & none & mother & 0.3 \\
\hline & male & 16 & 15 & 1.8 & 76 & ADHD & mother & 0.4 \\
\hline & male & 16 & 12 & 1.9 & 92 & ADHD & father & 2.4 \\
\hline & male & 15 & 14 & 2.1 & 94 & Autism & mother & 0.6 \\
\hline & female & 10 & 9 & 2.9 & 63 & none & mother & 0.6 \\
\hline
\end{tabular}


Analysis of the Wechsler subscales in all patients showed no significant difference between the average performal IQ (PIQ: 82) and the average verbal IQ (VIQ: 79). Kaufman's threefactor analysis of the Wechsler scales [Van Haasen et al., 1974] showed an average factorlIQ (verbal reasoning) of 79, factor2-IQ (visuo-spatial reasoning) of 82 and factor3-IQ (attention) of 81 . The differences between these three factors are not significant.

Longitudinal IQ data were available in two subjects. Results on the WISC-R in two children with juvenile DM showed a significant $(\mathrm{p}<0.05)$ decline of FSIQ over a period of 2 years (FSIQ's: from 94 to 71 and from 75 to 65 ).

\subsection{Behavioural and emotional functioning}

Results on the CBCL, parent form, (Figure 5) showed problem behaviour in the clinical range, in 5 of the 16 subjects: 1 of the 2 subjects with congenital DM and 4 of the 14 subjects with juvenile DM. Problems lay mainly in the internalising subscale of the CBCL: six subjects score in the clinical range on the internalising subscale, while only one subject scores in the clinical range on the externalising subscale. On the separate symptom clusters, the clusters "withdrawal" (three subjects), "social problems" (four subjects) and "attention problems" (two subjects) are in the clinical range ( $\mathrm{T}$-score $>70$, or above 98 th centile).

Two children scored in the clinical range of depression on the depression questionnaires: 1 girl with juvenile DM on the Children's Depression Scale, and 1 girl with congenital DM on the Beck Depression Inventory.

Figure 5 Scores on the different dimensions of the Child Behaviour Checklist (Parent's Questionnaire):

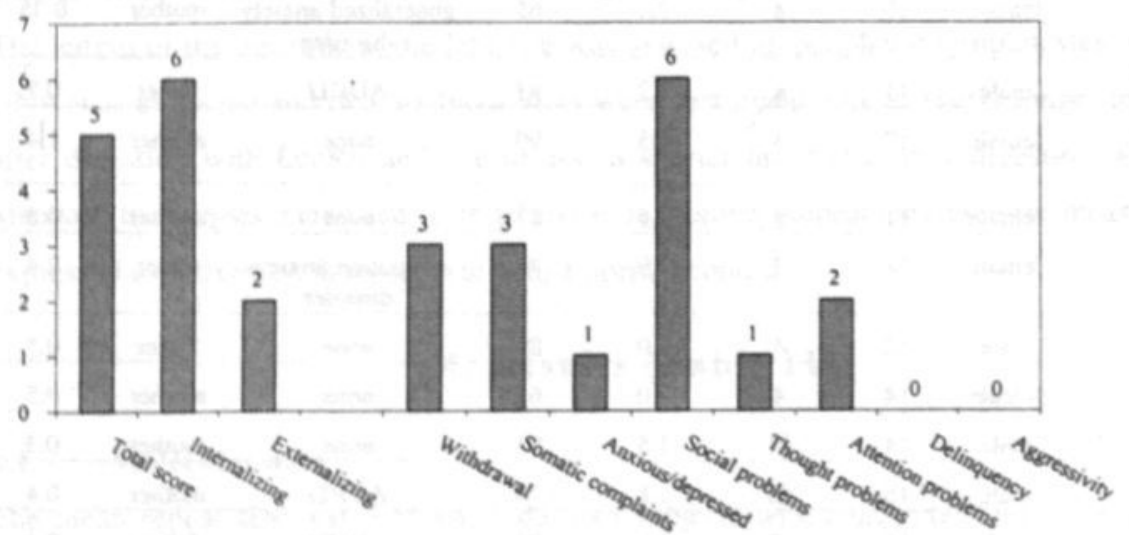

Bars represent the number of subjects scoring above +2 standard deviation of the mean. 


\subsection{Child psychiatric syndromes}

On the structured psychiatric interview, (ADIKA) nine subjects showed a clinical syndrome (Table 11). Four subjects fulfilled the criteria for Attention Deficit and Hyperactivity Disorder (ADHD): all four have juvenile DM and normal or borderline intelligence. The diagnosis ADHD was clinically diagnosed by the child psychiatrist in three of these four subjects. One of the females, with congenital DM, fulfilled the criteria for the diagnosis ADHD in childhood, but did not fulfil them anymore at the time of the study.

One mildly mentally retarded female with congenital DM and one borderline intelligent female with juvenile DM manifested a separation anxiety disorder before the age of 15 years. Finally, one mildly mentally retarded female DM had a generalized anxiety disorder and one borderline intelligent female had an adjustment disorder with depressive mood. These two subjects have juvenile DM.

One boy with normal intelligence and juvenile DM fulfils the criteria for autism on the Childhood Autism Rating Scale.

\section{Discussion}

We assessed sixteen children and adolescents with congenital or juvenile DM, and with intelligence levels in the normal, borderline or mildly retarded range.

In the two children with congenital DM, severe muscular hypotonia was present from birth on. In one subject, a foetal hypokinesia sequence with polyhydramnios was already noted in the third trimester of pregnancy. Despite these obvious symptoms, the diagnosis DM was only made at their respective age of 3 and 7 years. This long interval period between the age at onset of the first symptoms and the age at diagnosis may be explained by the fact that the two subjects were index-patients in their respective families. Further family studies showed that both inherited the DM mutation from their mother who presents a classical adult form of DM. In the 14 subjects with juvenile DM the mean age at diagnosis was 8.1. Although in these children other symptoms were present at the time of diagnosis, the first problem that alarmed the parents were learning difficulties. The fact that learning difficulties were the reason to seek help in these patients where DM was not known in the family may be the reason why the age at which the diagnosis was made coincides with the beginning of primary school.

In the patients we describe, within each category of DM, there is an important variation in severity of symptoms and affected organ systems within each diagnostic group. Repeat length does not permit to predict the variation within one diagnostic group, but there is a significant 
correlation between repeat length and FSIQ. In this study, we found a significant difference in repeat length between the diagnostic groups juvenile DM and congenital DM.

Moreover, further family studies showed that all affected parents ( 3 fathers and 8 mothers) presented a classical adult form of DM. As expected, the mean repeat size in the transmitting mothers was much smaller than in their affected children. Transmitting fathers had about the same repeat size as their affected children, although the differences in clinical severity between fathers and their children are important. Possible factors that may influence the phenotypic expression are somatic mosaicism, ongoing instability of the repeat expansion after birth, and the intrinsic characteristics of the protein kinase (DMPK) itself [Johnson K. and Siciliano, 1995].

On intelligence tests, all subjects in this study show a full scale IQ (FSIQ) below the population mean. The mean FSIQ of the group is 79. This result may not be representative for the whole population of DM patients, as in this group there was an ascertainment bias due to the fact that for several subjects the reason for consultation had been learning difficulties rather than somatic complaints. Previous studies performed in adult-onset DM showed higher mean FSIQ, ranging between 84 and 96 . These data are not very representative, as the subject group in these studies is always biased [Turnpenny et al., 1994]. In the present study, the subjects with congenital DM have a significantly lower FSIQ than the subjects with juvenile DM. As the subjects in the study are a group of referred patients, we cannot demonstrate whether the lower mean intelligence that was found, is due to ascertainment bias or reflects a truly lower intelligence in the population of children and adolescents with DM.

Several studies of cognitive functioning in adult DM have already demonstrated attention and concentration problems, and deficits in visuospatial and visuoconstructive abilities in affected individuals [Turnpenny et al., 1994]. However, some other studies showed hardly any evidence of cognitive dysfunction [Van Spaendonck et al., 1995]. In the present study of 16 affected children and adolescents, the profile of the intelligence test did not suggest specific cognitive deficits in the verbal or visuospatial area. However, more specific neuropsychological testing should be performed to assess this issue.

Longitudinal data on IQ evolution with age were available in two children and they showed a significant decline with age. Results of IQ evolution with age of several studies in adult-onset DM are controversial[Bird et al., 1983; Portwood et al., 1984; Portwood et al., 1986; Tuikka et al., 1993].

With regard to behaviour (results of the Child Behaviour Checklist, parents form) we found that an unexpectedly high number of children scored in the clinical range (five of sixteen 
children for total problem score). All these children are children with FSIQ $>70$ and, who are well in the range of intelligence for which the CBCL was designed. The pathological scores are mainly on the dimensions "withdrawal", "social problems" and "attention problems".

Two subjects scored in the clinical range on the Depression Scale we used. This incidence may not be very different from the incidence of depression in a normal population ( 2 to $5 \%$ ) and a mentally retarded population ( 8 to $15 \%$ ), according to the numbers of Lovell [Lovell and Reiss, 1993]. Both these children reported being teased at school.

A structured child psychiatric interview (ADIKA) showed Attention Deficit and Hyperactivity Disorder (ADHD) in four subjects at present, and in one more subject in the past. The diagnosis was made in three of these subjects at clinical assessment by a child psychiatrist. The discrepancy between the higher number of subjects, who fulfilled the diagnostic criteria of the DSM-III-R (ADIKA) and the somewhat lower number diagnosed by the child psychiatrist, can be due to the fact that the subjects mainly present symptoms relating to attention deficit and impulsivity, and fewer relating to hyperactivity. This may lead to the child psychiatrist not concluding to the presence of the diagnosis, although the criteria are fulfilled. The prevalence of ADHD in a population with intelligence higher than 70 is estimated to be between 1 and 3\% [American Psychiatric Association, 1994]. In our sample, the prevalence was $25 \%(4 / 16)$. Besides the subjects in whom the full diagnosis of ADHD was made, attention problems were one of the most frequent complaints of the parents in the other subjects. Thus, attention problems, with or without hyperactivity and impulsivity seem to be frequent problems in the population of children with DM. It is not clear how much somnolence or problems of circadian rhythm in DM contribute to the attention problems in DM.

Another frequent diagnosis was that of anxiety disorders (three in sixteen subjects).

In two adolescents (> 16 years, 2 juvenile DM) reduced initiative was reported and they presented more pronounced muscle weakness. In the other fourteen subjects, there were no complaints that could refer to the presence of apathy. Apparently, the clinical diagnosis of "apathy" which is made in many DM children may be a false impression caused by their expressionless face.

In conclusion, the observation made in the present group of $16 \mathrm{DM}$ children and adolescents are interesting and reveal an unexpected incidence of different child psychiatric syndromes (ADHD, anxiety disorders). 


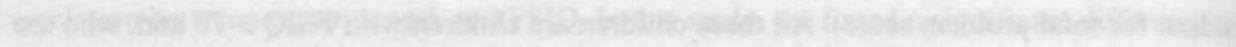

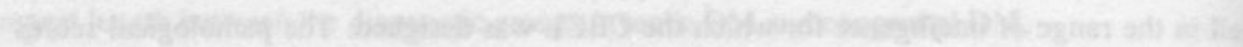

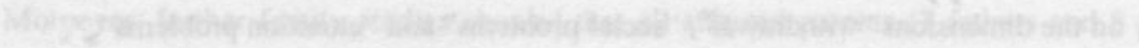

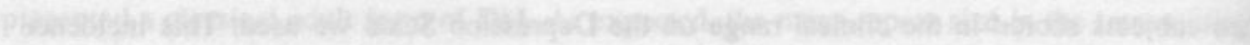

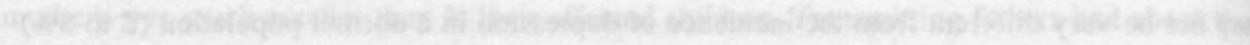

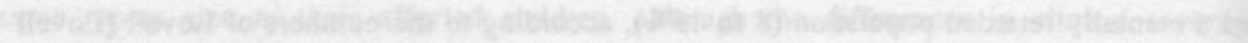

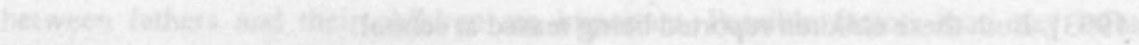

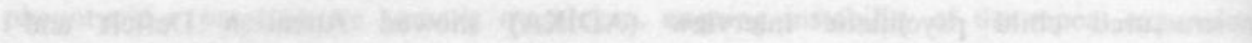

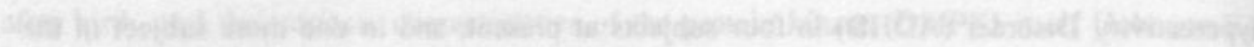

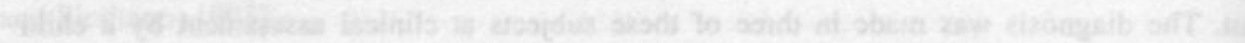

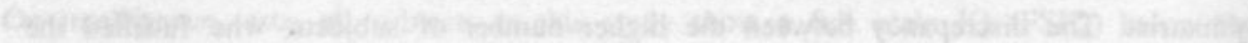

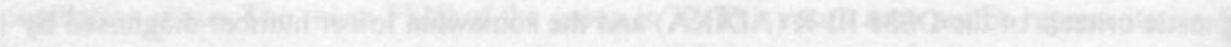

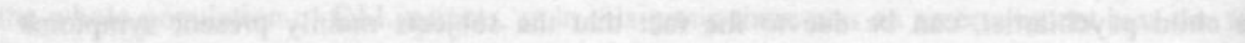

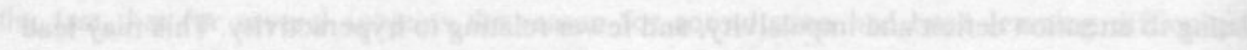

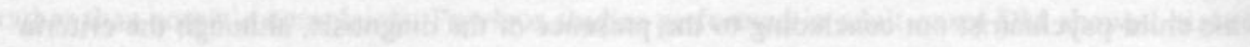

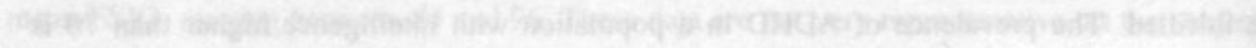

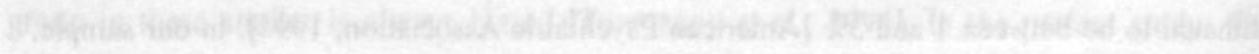

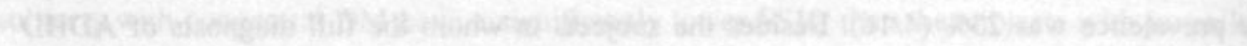

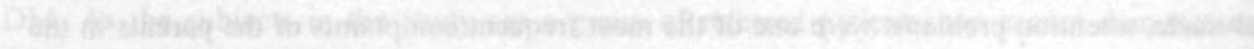

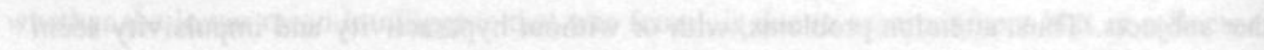

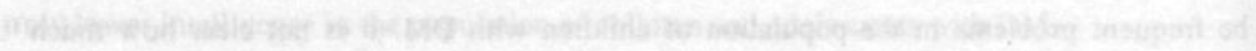

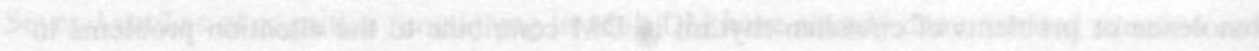

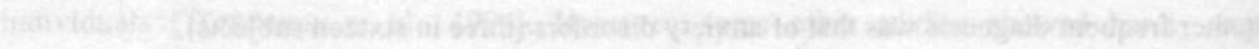

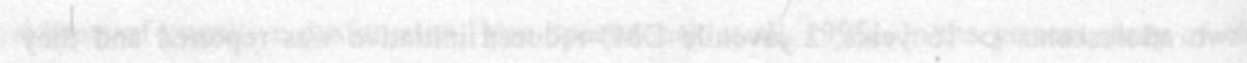

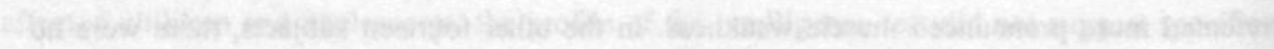

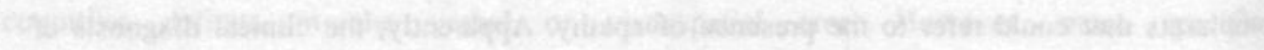

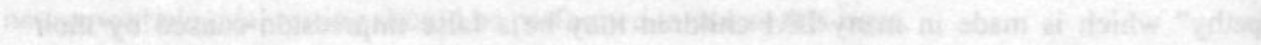

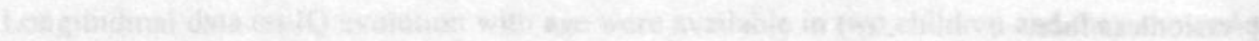

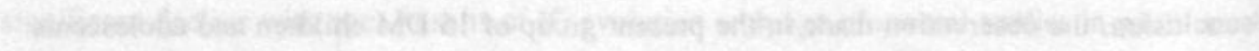

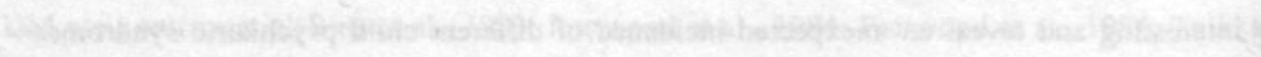

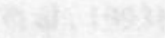

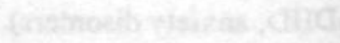
SWati

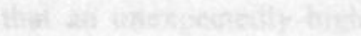




\section{Chapter 10 Emotional and Behavioural profile and Child Psychiatric Diagnosis in the Childhood Type of Myotonic Dystrophy.}

Goossens $E^{1}$, Steyaert J ${ }^{2,3}$, de Die-Smulders $C^{3}$., Willekens $D^{2}$, Fryns J.-P. ${ }^{2}$. (2000)

' Department of Child and Adolescent Psychiatry, University of Leuven, Belgium

${ }^{2}$ Centre for Human Genetics, University of Leuven, Belgium

${ }^{3}$ Department of Clinical Genetics, Academic Hospital Maastricht, the Netherlands 


\section{Abstract}

Emotional and behavioural profile and child psychiatric diagnosis in the childhood type of myotonic dystrophy: We report data on behaviour and possibly associated psychopathology in 24 children and adolescents between 9 and 22 years of age, with the childhood type of myotonic dystrophy. The patients were recruited through a patients association, as family members from patients with myotonic dystrophy (DM). In $41 \%$ of the subjects, a score in the clinical abnormal range was found on the Child Behavior Checklist (completed by parents and by teachers). In $62 \%$ of the subjects, a child psychiatric diagnosis was found using a standardised psychiatric interview. The most frequent child psychiatric diagnoses were Attention Deficit with Hyperactivity Disorder and Anxiety Disorder. 


\section{Introduction}

Myotonic dystrophy (DM) is an autosomal dominant, neuromuscular disease, characterised by myotonia and progressive muscular weakness. Other symptoms can be present depending on the affected organ system: smooth muscle disturbances (swallowing problems, spastic colon. diarrhoea), cataract, gonadal dysfunction in males (subfertility), cardiac conduction defects. In adults hypersomnia, loss of initiative and apathy are among the most common systemic complications. When the disease starts at young age, mental retardation, usually mild to moderate in degree, is the rule. DM is caused by an expanded and unstable CTG repeat sequence in the $3^{\prime}$ untranslated region of the DMPK gene on the long arm of chromosome 19 [Harper, 1998]. Four disease types can be distinguished based on the age of onset and predominant symptoms [Harper, 2001]: (1). The mild type with cataract and minimal or no neuromuscular symptoms in middle or older age. (2). The classical or adult type with neuromuscular symptoms in adolescence or early adult life (age at onset 12-50 years). (3). The childhood or juvenile type with symptoms before the age of 12 years and an uneventful pre- and neonatal history and normal development within the first year of life. Learning disabilities are often prominent, while neuromuscular symptoms are rather mild or sometimes even absent [de Die-Smulders C., 2000]. (4). The congenital type with clinical symptoms present in utero (reduced foetal movements and history of polyhydramnios) or from birth (hypotonia, facial diplegia, often skeletal deformities, respiratory and/ or feeding problems). Mild to moderate developmental delay is seen in survivors.

Psychopathology in patients with myotonic dystrophy has been documented in several studies. In adults a variety of problems was described, such as a high incidence of avoiding personality, and a medication-responsive major depressive disorder [Brumback and Carlson, 1983; Colombo et al., 1992; Delaporte, 1998]. However, others did not find anxio-depressive symptomatology or other significant psychiatric morbidity [Bungener et al., 1998; Cuthill et al., 1988]. Disturbances of mood and affect, a depressive attitude with a somatic concern, difficulties in social life or an emotional deficit and changes in character are also described [Bungener et al., 1998; Cuthill et al., 1988; Franzese et al., 1991]. Ambrosini and Nurnberg [Ambrosini and Nurnberg, 1979] mentioned clinical evidence for an altered mental functioning as a basic feature of DM, rather than a reactive or secondary phenomenon, due to a possible difficult adaptation to the threatening implications of the disease. Their hypothesis was that thalamic dysfunction causes the (neuro)psychopathologic changes (e.g. changes in diurnal rhythm, narcolepsy, hypersomnia, disorientation, confabulation, somnolence and 
akinetic mutism). Primary cerebral involvement in DM patients was confirmed by the presence of abnormalities in neuro-imaging studies, such as cerebral atrophy and focal white matter lesions [Ashizawa, 1998; Censori et al., 1994; Damian et al., 1994a; Damian et al., 1994b]. Some cerebral lesions seem to be characteristic of the disease, while others are similar to the age related alterations [Censori et al., 1994]. Damian et al. [Damian et al., 1994b] concluded that the cognitive impact of brain lesions depends on the distribution as well as the total extent of white matter lesions, e.g. subcortical lesions have more impact on cognitive functioning than periventricular lesions and are responsible for attention problems, not for deficit in intelligence. Furthermore cerebral hypoperfusion on single photon emission computed tomography (SPECT) and an altered cerebral metabolism have been documented [Chang et al., 1993; Chang et al., 1998; Fiorelli et al., 1992]. Neuropathological examination of brains of adult DM patients found intracytoplasmatic inclusions, neurofibrillary tangles containing pathological tau protein and neuronal loss [Ashizawa, 1998]. In affected children the central nervous manifestations may be much more conspicuous than those seen in adults, as mental retardation is a major feature in the congenital as well as in the childhood type [Ashizawa, 1998; de Die-Smulders C., 2000]. Although serious anatomical brain defects are not a constant finding, ventricular dilatation and hypoplasia of the corpus callosum were found in an MRI study on congenital DM [Hashimoto et al., 1995] and malformations of the cerebral cortex were reported in a neuropathological study of two patients with presumed childhood onset DM [Rosman and Rebeiz, 1967]. However, psychopathology in DM affected children and adolescents is scarcely documented. Blondis et al. [Blondis et al., 1996] described a 10 years old DM affected girl with Asperger syndrome. In a pilot study, Steyaert et al. [Steyaert et al., 1997] showed that more than half of the children with childhood onset DM shows signs of psychopathology and that most subjects have some form of cognitive impairment. The present study is an extension of the latter study. It is part of a research project where we assess the clinical features, cognitive functioning, neuropsychological impairments and psychopathology in children with childhood DM [de Die-Smulders C., 2000].

\section{Subjects and methods}

\subsection{Subjects}

We studied a group of 24 children and adolescents, between the age of 9 and 22 years, 14 boys and 10 girls. They were children of members of the Dutch patient organisation for 
Myotonic Dystrophy patients and were recruited by way of a call for patients. They were included in the study on the following basis: clinical diagnosis of DM with onset between 1 and 12 years, and learning difficulties, severe enough to cause failure at primary school level, as well as absence of congenital features. All subjects had been diagnosed with DM prior to the study. The diagnosis of DM was confirmed by molecular studies. In most participants the parents worried about possibly neurological and or learning problems in their child. The patients came from 23 families (one sibpair). Twenty one of the subjects attended school: 4 subjects were in a normal school programme, 17 subjects were in a special education programme (11 for the mildly mentally retarded, 3 for children with specific learning disabilities, 2 for motor disabled persons and 1 for moderate mental retarded children). One subject works in a sheltered workshop.

The subjects were tested with the Wechsler Intelligence Scale for Children, revised version (WISC-R) [Vander Steene et al., 1986] or the Wechsler Adult Intelligence Scale (WAIS) [Stinissen et al., 1970] in the adolescents older than 16 years. Full scale IQ (FSIQ) varied between 50 and 97 . The mean FSIQ is 72.25 with a SD of 14. There is no significant difference between verbal and performance IQ.

Data on the transmitting parent's clinical type of DM could be obtained for all our subjects. Twenty-two DM parents had the adult type of DM, one had the mild type and one was free of symptoms. In thirteen subjects the mother was the transmitting parent and in 11 subjects it was the father. Molecular data on the transmitting parent were available for 13 families.

\subsection{Methods}

The Dutch versions of Achenbach's Child Behavior Checklist (CBCL) and the coupled Teacher Report Form (TRF) [Verhulst F.C. et al., 1996] are well-validated checklists to assess behaviour and emotional problems in children. These checklists have been standardised for children between 4 and 18 years old. The parents complete the CBCL; the teacher completes the TRF. In this study the CBCL was completed by the parents in all subjects. A TRF was completed by the teacher in 13 of the 24 subjects.

The results of these checklists are transformed into a total problem score and into two subscores for, respectively, the internalising and externalising problem behaviour. This total problem score and the two subscores are transformed into T-scores. A T-score higher than 63 ( $>90$ th centile) is found to discriminate between normal children and children with emotional and/or behaviour problems who would benefit from care. A T-score of 70 or higher ( $>97$ th 
centile) is found in children with severe emotional and/or behavioural problems (30). The results are also transformed into eight clusters of specific problem behaviour: withdrawn, somatic complaints, anxious/depressed, social problems, thought problems, attention problems, delinquent behaviour and aggressive behaviour. Each of these are transformed into a T-score $($ mean $=50$, S.D. $=10)$. A T-score higher than $67(>95$ th centile $)$ is considered to be of concern. A T-score of 70 or higher ( $>97$ th centile) is considered to be in the clinical abnormal range.

A structured child psychiatric interview with the parents has been used to find out a possible child psychiatric syndrome. We used the Dutch version (ADIKA [Kortenbout van der Sluijs et al., 1993]) of the Diagnostic Interview for Children and Adolescents (DICA [Reich et al., 1991]). This interview is based on the criteria of the DSM-III-R classification [American Psychiatric Association, 1987]. As the DSM-III-R criteria for the diagnosis Attention Deficit Disorder with Hyperactivity (A.D.H.D.) are quite different from the DSM-IV criteria for A.D.H.D [American Psychiatric Association, 1994], we used an adaptation of the ADIKA [Célistin-Westreich and Ponjaert, 1998] that includes the new DSM-IV criteria. For the diagnosis of autism, which is not included in the structured interview, we adapted the ADIKA, based on the DSM-IV criteria.

Differences between intelligence scales were assessed using Student's t-test. The probability of finding a particular number of child psychiatric diagnoses or abnormal findings on the scales of the CBCL was estimated using a test for probability of binomial distributions. For each measured variable, the number of abnormal findings was compared to the expected proportion of abnormal findings in the general population, e.g. when we used the cut-off for abnormality at the 95th centile in the CBCL, the findings in the studied subjects were compared to a frequency of abnormality of $5 \%$. Pearson's correlations were used to compare the continuous variables "repeat length in child". "Full Scale IQ" (FSIQ), and "CBCL Total Problem Score".

Two procedures were used to assess the possible effect of the transmitting parent's sex on the presence of behaviour problems. (1) For the categorical data in the child psychiatric interview (ADIKA): as the number of subjects is small, the effect of the transmitting parent's sex on the type of psychiatric problems cannot be statistically assessed, and thus for analysis we dichotomised the results of the ADIKA in "psychiatric problems present" and "psychiatric problems absent". Using the Pearsons Chi-square test this dichotomous variable was correlated with the dichotomous variable "transmitting mother" versus "transmitting father". (2) The effect of the transmitting parent's sex on the continuous variables "Full scale IQ" and 
"Total problem score on the Child Behaviour Checklist" were assessed by means of ANOVA and ANCOVA analysis. In both assessment techniques, the effect of the clinical type of DM in the transmitting parent could not be assessed, as the number of "mild type" parents was only two.

\section{Results}

\subsection{Behavioural and emotional problems: CBCL (Table 12)}

Using the $\mathrm{CBCL}$, we found a total problem score in the clinical range in $10 / 24$ subjects (42\%). This is significantly ( $p<0.001)$ above the expected number of subjects considering that we set the threshold at the 90 th centile, as recommended by Verhulst et al. [Verhulst F. C. et al., 1990]. Nine subjects $(37.5 \%)$ score in the clinical range for internalising problems ( $p<$ $0.001)$ and 2 subjects $(8 \%)$ score in the clinical range for externalising problems $(p=0.564)$.

Table 12 Results of the CBCL and TRF.

\begin{tabular}{|c|c|c|c|c|}
\hline & \multicolumn{2}{|c|}{ Parents' report (CBCL) } & \multicolumn{2}{|c|}{ Teacher's report (TRE) } \\
\hline & $\begin{array}{l}\text { Number in } \\
\text { clinical range } \\
(n=24)\end{array}$ & $\begin{array}{l}\text { Probability to } \\
\text { find this } \\
\text { prevalence }\end{array}$ & $\begin{array}{c}\text { Number in clinical } \\
\text { range }(n=13)\end{array}$ & $\begin{array}{l}\text { Probability to find } \\
\text { this prevalence }\end{array}$ \\
\hline Main scales & 1 & xyfority & & \\
\hline Total problem & 10 & $<0.001$ & 3 & 0.134 \\
\hline Internalising & 9 & 0.003 & 5 & 0.006 \\
\hline Externalising & 2 & 0.564 & 0 & 0.254 \\
\hline \multicolumn{5}{|l|}{ Problem domains } \\
\hline Withdrawn & 7 & $<0.001$ & 3 & 0.024 \\
\hline Somatic Complaints & 9 & $<0.001$ & 4 & 0.003 \\
\hline Anxious Depressed & 3 & 0.116 & 3 & 0.024 \\
\hline Social problems & 13 & $<0.001$ & 5 & $<0.001$ \\
\hline Thought problems & 6 & $<0.001$ & 2 & 0.135 \\
\hline Attention problems & 9 & $<0.001$ & 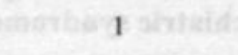 & 0.487 \\
\hline $\begin{array}{c}\text { Delinquent } \\
\text { behaviour }\end{array}$ & 1 & 0.661 & 0 & 0.513 \\
\hline $\begin{array}{r}\text { Aggressive } \\
\text { behaviour }\end{array}$ & 2 & 0.339 & 0 & 0.513 \\
\hline
\end{tabular}

Number of subjects with a score in the clinical range for the several scales and problem domains of the Child Behaviour Checklist and Teacher Report Form of the CBCL 
On the Teacher's Report Form (TRF) of the behaviour checklist, 3 of 11 subjects (27\%) had a total score in the clinical range ( $p<0.09), 5$ others $(45.4 \%)$ had an internalising score in the clinical range ( $p=0.003)$ and none of the subjects had a score for externalising problems in the clinical range. The specific problem scales show that the subjects have most problems in the areas "withdrawn", "social interaction" and "inattention" (Table 12). Nine children also scored in the clinical range in the problem domain "somatic complaints".

No correlation between FSIQ and total problem score on the CBCL could be demonstrated: Pearson's correlation between the two continuous variables was 0.111 ( $p=0.607$ ). Nor was there a significant correlation between total problem score on CBCL and CTG repeat length of the children: Pearson's correlation 0.158 ( $\mathrm{p}=0.484$ ).

Table 13 Child psychiatric diagnoses (ADIKA).

\begin{tabular}{lc}
\hline Diagnosis on ADIKA & $\begin{array}{c}\text { Number of subjects with } \\
\text { this diagnosis }(\mathbf{n}=\mathbf{2 4})\end{array}$ \\
\hline No diagnosis & 9 \\
\hline Anxiety disorders combined & 6 \\
Separation anxiety disorder & 4 \\
Generalised anxiety disorder & 1 \\
Simple phobia & 1 \\
Altention Deficit Hyperactivity Disorder & 8 \\
Dysthymic disorder & 2 \\
Encopresis & 1 \\
Enuresis & 1 \\
Adjustment disorder & 4 \\
\hline
\end{tabular}

Number of children with a particular diagnosis on the child psychiatric interview ADIKA. A number of children have more than one diagnosis. For anxiety disorders, the numbers for the subtypes separately are given below the total number.

\subsection{Child psychiatric syndromes}

Using the ADIKA we found out that 15 of the 24 subjects $(63 \%)$ had a child psychiatric diagnosis (Table 13), and 11 subjects had no psychiatric diagnosis. Eleven subjects (45.8\%) had one diagnosis, three subjects had 2 diagnoses and 1 subject had 4 diagnoses. Looking at the whole group, we found Attention Deficit Hyperactivity Disorder (ADHD) in eight subjects $(33 \%): 6$ have the combined type, 2 have the predominantly inattentive type. Two subjects $(8 \%)$ had symptoms of ADHD but did not fulfil the diagnostic DSM-IV criteria for 
duration or for age of onset. In six subjects $(25 \%)$ we found an anxiety disorder as first or as second diagnosis: separation anxiety disorder in 4 , generalised anxiety disorder in 1 and specific phobia in 1 . In two subjects $(8.3 \%$ ) a mood disorder was found as first or as second diagnosis. In one subject $(4 \%)$ we found encopresis as the fourth diagnosis and in one subject (4\%) we found enuresis as the fourth diagnosis. Four subjects $(16 \%)$ have signs of an adjustment disorder.

\subsection{Effect of transmitting parent}

The thirteen $(54 \%)$ children where mother was the transmitting parent had an mean CTG repeat length of 752 triplets ( $s d=141)$, and a mean FSIQ of $67(s d=12.1)$. The eleven $(46 \%)$ where father was the transmitter had a mean CTG repeat length of 517 triplets (sd=143), and a mean FSIQ of 79 ( $\mathrm{sd}=13.3$ ). ANOVA analysis showed a significant effect of the transmitting parent's sex on the Full Scale IQ of the affected child $(\mathrm{p}=0.034)$. However, the effect of the transmitting parent's sex disappeared as separate predictor of the children's FSIQ when the childrens' repeat length at the DM site was taken into account as a co-variable in ANCOVA analysis. This ANCOVA model yielded an $F=0.052(p=0.822)$ for transmitting parent's sex and $F=6.065$ ( $p=0.024)$ for the co-variate "child's repeat length". There is a high correlation between the transmitting parent's sex and the child's repeat length (separate ANOVA: $\mathrm{F}=14.640, \mathrm{p}=0.001$ ).

The same analysis was performed for transmitting parent's sex and the Total Problem Score on the Child Behavior Checklist. This yielded no association between both variables: ANCOVA effect of transmitting parent $\mathrm{F}=0.181$ ( $\mathrm{p}=0.675$ ) and effect of the co-variate "child's repeat length" $F=0.065(\mathrm{p}=0.802)$.

Pearson's chi-square to test the effect of the transmitting parent's sex on the presence of child psychiatric pathology on the ADIKA yielded no significant correlation: chi-value 0.906 (1 d.f.; $p=0.341$ ).

\section{Discussion}

We studied a group of 24 children and adolescents with the childhood type of DM. As this category of DM patients is not known to have important neurological symptoms or motor impairment, the subjects in this study were not clinical patients. We looked at their intelligence, their level of behaviour and/or emotional problems, and finally at the absence or presence of diagnosable child psychiatric syndromes. 
Though most subjects had only minimal neurological signs, their mean full-scale intelligence quotient (FSIQ) was well below normal. Lower FSIQ was correlated with longer repeat lengths, which in turn correlated with the transmitting parent's sex: inheritance from mother gives longer repeat lengths. However, the data suggest that there is no independent effect from the transmitting parent's sex on the FSIQ of the affected child, and that lower FSIQ is mediated through longer repeat lengths when the child inherits the DM expansion from his mother.

The Child Behaviour Checklist showed that an unusual number of children have behaviour and/or emotional problems in the clinical range. This means that the psychological development of many of these children is of concern. A correlation between DM repeat length and problem scores could not be demonstrated. Neither could we find a correlation between the transmitting parent's sex and the problem scores.

We found an important number of subjects with a child psychiatric diagnosis, mostly ADHD and Anxiety Disorder. ADHD has a significantly high prevalence in this group, considering that in DSM-IV [American Psychiatric Association, 1994] the prevalence of ADHD in the general population is estimated between 2 and $5 \%$ ( $<<0.001$ for a population prevalence of $5 \%$ ). The statistical significance of the number of children with anxiety disorders in the DM group is not clear, as the prevalence of anxiety disorders in children is not well documented. However, the prevalence of $25 \%$ in this childhood type DM group is likely to be unusually high. The presence or absence of a child psychiatric syndrome was not associated to the transmitting parent's sex.

With this larger group we confirm the findings of Steyaert et al. [Steyaert et al., 1997], who documented an unexpected prevalence of psychiatric disorders in children with DM. Van Spaendonck et al. [Van Spaendonck et al., 1995] concluded that there is evidence for poor cognitive control of behaviour in adult patients with DM, and attention deficits and vigilance problems are frequent problems in subjects with an early onset type of DM [Harper, 2001]. Therefore, it is not unexpected to find ADHD in children and adolescents with DM, as attention problems and poor cognitive control are key features of ADHD. We did not observe a significantly higher prevalence of mood disorders in children and adolescents with childhood type DM, in contrast with the finding of Cuthill et al. [Cuthill et al., 1988] that adults with DM having more depressions.

Not much is known about the causal mechanisms of psychopathology in children with DM, and this study falls short of clarifying these. Three hypotheses can be suggested: (1) DM is a brain disorder that affects brain functioning directly - e.g. at the molecular or structural level - 
and leads to psychological development problems; (2) children have a reactive maladjustment to the fact that they have a chronic an progressively impairing condition; this leads to behaviour and/or emotional problems; (3) a transmitting and affected parent has poor parenting skills and this affects the child's development.

Three arguments are in favour of the hypothesis of a brain disorder leading to developmental problems. The first is that brain-imaging studies demonstrated functional brain anomalies in congenitally affected children and adults with DM [Ashizawa, 1998; Hashimoto et al., 1995]. One may assume that these brain anomalies are also present in childhood onset DM. The second argument is that as a group, children with childhood onset DM have a significantly lower intelligence level. As they do not present significant motor impairment in childhood, it is likely that the lower IQ has a primarily central origin. A third argument is the high prevalence of ADHD in these children. ADHD is generally considered as a form of psychopathology arising from a dysfunction in brain development rather than as a reactive pattern of psychopathology.

The "reactive maladjustment" hypothesis is less probable, as the cognitive and behavioural symptoms often occur well before the diagnosis has been made, at a time that the physical signs are mild or absent and the children have no muscle impairment. Thus, it is unlikely that their neurological condition would be a stressor at this stage. And though their lower intelligence level and learning disability can be a cause of stress, the number of child psychiatric conditions is unlikely high even for a group of children with borderline IQ or a mild learning disability.

In the present study there are no strong arguments in favour of the "poor parenting" hypothesis. If poor parenting by an affected parent would have a major effect on the child's psychological functioning, one might see that the degree of psychological dysfunction would be dependent on whether mother or father is affected. In the present study we found that full scale IQ is correlated to the transmitting parent's sex, but that this effect can almost completely be attributed to the effect of the transmitting parent's sex on the DM repeat length in the child. Total problem score on the Child Behaviour Checklist, and presence of child psychiatric syndromes did not correlate with the DM repeat length, nor did these correlate with the transmitting parent's sex. This suggests that other factors, combined with the expanded DM repeat, are necessary for children with childhood type DM to develop clinically significant behaviour and/ or emotional problems, while having a low intelligence is directly associated with a longer repeat length. 
In order to clarify further the mechanisms of psychopathology in children with DM, it will be necessary to analyse the psychopathology in these subjects more thoroughly, including using unaffected siblings as controls. In this perspective an important question is which central neurological differences exist between DM subjects with different psychopathology, or without problems. A first hypothesis is that in DM, there is a progressive dysfunction of at least the frontal lobes. This could explain both the apathy in advanced DM subjects and the problems of impulse inhibition and attention seen in the young patients with childhood DM and ADHD.

In conclusion, the results of this sample of 24 children and adolescents with childhood onset DM reveal an unexpected high incidence of different child psychiatric syndromes (mainly $\mathrm{ADHD}$ and anxiety disorders). Although we do not know how representative this sample is for the whole population of children with DM, it seems likely that children with childhood type DM are a group with important risks for emotional and behavioural problems. This study also underlines the importance of accurate neurological and/or genetic investigation of children, admitted to child and adolescent psychiatry units with learning disabilities, ADHD, or other behavioural and/or emotional problems. Finding the underlying somatic diagnosis may have major implications for their treatment and long-term prognosis. 
Part III. GENERAL DISCUSSION 


\section{Chapter 11 Single gene disorders and behavioural phenotype}

\section{The problem of complexity}

In the vast amount of ongoing research and discussion on the relationships between genes and behaviour, single gene disorders are a challenging opportunity to understand at least some of the mechanisms involved in the genetic aspects of neuropsychiatric conditions. These conditions are likely to be influenced by many genetic and non-genetic factors. Ideally, the gene-behaviour relationships in these conditions should be analysed with models that take into account multiple genes and both genetic and non-genetic factors. Using such models, behaviour sciences and quantitative genetics have generated interesting findings regarding the hereditary and environmental influences in neuropsychiatric conditions [Plomin et al., 2000; Rutter et al., 1999a, b]. However, the study of the molecular biology of these conditions is accompanied by major difficulties. The first problem is the phenotype itself $(0)$. There is no good evidence that the way neuropsychiatric disorders are conventionally delineated is an effective entry into the molecular research of these disorders. E.g. all individuals with autism fulfil the current diagnostic criteria for the disorder. However, they show a wide variation in cognitive and behavioural characteristics and family history. The clinical differences are so large, that it is unlikely that they reflect between-subject variation of one disease. It is more probable that in autism we are confronted with different phenotypes, with different underlying neuro-psychopathological pathways, each with quite different (genetic and other) causes. Teasing apart these phenotypes may be necessary to refine molecular research. A second problem is that the molecular biology of a multifactorial polygenic model is extremely complex. We do not (yet) have the methods to analyse them, and often the knowledge of the molecular pathways to which the candidate genes belong is insufficient. This is like sorting out a huge jigsaw puzzle without the example, while knowing that many pieces are missing. A third problem is that knowledge on the developmental trajectories of brain and mind and how genes affect it is still very limited. Consequently, integrating this essential factor of development in multifactorial polygenic models would lead to even more terra incognita. Omitting it on the other hand, undermines the credibility of the proposed models. In fact, this is a circular problem of the chicken-or-egg-first type which can only gradually been solved by 
combining the fields of genetic and developmental research: genetic research helps to understand development; knowledge on development helps to find the role of genes

\section{The manageable study of single genes}

These different obstacles urge us to be humble and study the manageable rather than the impossible. In this thesis, I advocated that the collaboration between clinical genetics and molecular biology in research on chromosomal defects and single gene disorders shows behavioural geneticists a path to follow. Through accurate clinical observations, phenotypes of disorders like Fragile X Syndrome could be described and delineated $(0)$, which helped molecular geneticists to find the causal genes. Once a gene has been found, the mechanisms and pathways between defect and phenotype can be analysed for that particular gene. In its turn, this has led to completely new insights, like the discovery of dynamic mutations $(0)$ and the variable effect that they have on the phenotype ( 0 Fragile X Syndrome; 0 Myotonic Dystrophy). Other mutations have a variable penetrance, that can depend on stochastic factors, and/or the effect of other genes ( 0$)$. In some mutations the effect of other genes is gradual and either small (e.g. Fragile X syndrome) or large (e.g. Velo-cardio-facial syndrome). In other mutations, the effect of other genes is discontinuous and a necessary condition for the expression of a mutation (e.g. in Bardet-Biedl syndrome) (0). Research on the Fragile $\mathrm{X}$ and Myotonic Dystrophy mutations have demonstrated that not only deficiency of gene product, but also overproduction of mRNA can have pathological effects and explain some aspects of the phenotype.

All these mechanisms that cause variation in the molecular pathway between gene and phenotype are no exceptions. Recent history of the discovery of single genes shows that after initial detection, subsequent research often reveals the complexity of the expression of the gene. Consequently, this complexity in the expression of individual genes should be taken into account when one tries to reconstruct biological pathways in which sets of genes and their gene products participate, as one does when putting a number of candidate genes in a molecular model to explain a particular neuropsychiatric condition, e.g. in presenile Alzheimer Dementia [Theuns and Van Broeckhoven, 2000] or Tourette Syndrome [Comings et al., 1999].

The fact that research on single genes is of manageable complexity has made it possible to get better insights not only in molecular biology, but also in some aspects of brain development. Research on brain development in some forms of nonspecific X-linked mental retardation due to single gene mutations, has led to the finding of defects in signal- transduction pathways 
that are believed to function during neuronal maturation. These findings provided insights into molecular and cellular defects that underlie mental retardation. [Chelly and Mandel, 2001; Frints et al., 2002].

Similarly, single gene disorders can form a starting point to study the interaction between environmental factors and gene expression. To our knowledge, research in this field is still very limited. 


\section{Chapter 12 Dynamic mutation disorders}

\section{Dynamic mutations}

The typical phenotype of Fragile X Syndrome had been known since more than a decade when the mutation in the promoter region of the FMRI-gene was found $(0)$. The discovery of a new type of mutation, a trinucleotide repeat expansion [Verkerk et al., 1991] was a milestone. Since then, at least a dozen mutations of this type, now called dynamic mutations, have been discovered. All of them are in genes that are mainly expressed in the central nervous system. The significance of this is unclear.

The core characteristic of dynamic mutations is the variable length of the mutation $(0)$. In this thesis we reviewed the research on genotype-phenotype relations in dynamic mutations. Different mutation lengths can be associated with different clinical characteristics. In some disorders, e.g. Huntington's chorea and Myotonic Dystrophy, longer expansions are associated with an earlier age of onset of the disease. In other dynamic mutation disorders, e.g. Fragile X syndrome and Spinocerebellar Ataxia (SCA8-gene), there is evidence that very different expansion lengths are associated with a qualitatively different phenotype.

\section{Fragile X Syndrome}

One of the consequences of the discovery of the "marker X chromosome" in Fragile X syndrome (FXS) [Lubs, 1969; Sutherland, 1977], was that carriership (of what was later found to be the full mutation) could be demonstrated in family members who did not show the typical Fragile X phenotype, or who were clinically unaffected. Detection of the "marker X chromosome" in males with FXS made it possible to determine which mothers were obligate carriers, even if the "marker X chromosome" could not be found in these female subjects. We examined 11 female obligate carriers who did not show the typical Fragile $\mathrm{X}$ phenotype (0). Subjects of at least normal intelligence were chosen for this study, and we demonstrated that even under that condition a higher than expected number of subjects shows academic problems and/or specific cognitive weaknesses. At the time of this study, we did not have the opportunity to study further the molecular characteristics of the FMRI-gene in these subjects. 
The discovery of the trinucleotide repeat expansion and the development of the DNA-test to detect it, made the problem of the variance in phenotype even more complex. Beside mentally retarded carriers of the full mutation, carriers of the full mutation who did not display the typical Fragile X phenotype, and carriers of the premutation could be detected from then on. We studied two different aspects of the genotype-phenotype correlation in subjects who do not display the typical Fragile $\mathrm{X}$ phenotype.

The first of these two studies (Chapter 7) focused on males with FXS. Beside the majority of males with the typical FXS, there is a minority of subjects who function markedly better. One possible cause is mosaicism: subjects in whom a fraction of the cells carries the full mutation, and a fraction the premutation. Another cause is incomplete hypermethylation of the CpGisland in the promoter region of the FMRI-gene. In the full mutation this CpG-island is normally hypermethylated. We described seven boys with a full mutation, but incomplete methylation of the CpG-island and demonstrated that the full scale IQ correlates inversely with the percentage of hypermethylation of the CpG-island but not with the CGG repeat length. This provided clinical evidence that hypermethylation of the $\mathrm{CpG}$ island is an important element in the phenotype. It has been shown that unmethylated CGG repeats in the full mutation range cause slower transcription to mRNA, but do not completely block it [Feng et al., 1995]. Hypermethylation of the CpG island blocks transcription of the FMRI-gene. Unfortunately, we did not have the opportunity to measure the FMR1-protein levels in these subjects.

The second study focused on female premutation carriers (Chapter 7). The finding that women with the fragile $\mathrm{X}$ premutation have an unexpectedly high prevalence of premature ovarian failure (POF) [Allingham-Hawkins et al., 1999], showed that the phenotype is not necessarily normal in the premutation, as had been assumed before. We found minor neurocognitive anomalies in a minority of female premutation carriers, that none of the control subjects displayed, neither any of the other premutation carriers. There was a bimodal distribution within the group of premutation carriers. Although performed in a completely different area than the POF-study, our finding is in line with it: we also found an abnormal phenotype in about one in five female premutation carriers. This suggests that factors other than the fragile $\mathrm{X}$ premutation have an additional effect on anomalies in the premutation phenotype. Increased levels of FMRl-mRNA have been observed in some premutation subjects and could be one such factor. FMRP-levels are generally normal in premutation subjects. In the subjects of this study, we did not have the opportunity to measure FMRP and FMRI-mRNA levels. 
The existence of a phenotype associated with the fragile $\mathrm{X}$ premutation needs further confirmation. Unlike the problem of possible POF, the observed neurocognitive characteristics do not seem to cause significant impairment in premutation carriers. However, the observed characteristics are a sign that "something different" happens in the neurons of the affected subjects. Further research on the molecular mechanisms implicated here may give new insights in the functioning of the FMRI-gene and its product, and on how a mutation in one gene may affect a whole range of intracellular pathways.

\section{Myotonic dystrophy}

Myotonic Dystrophy (Dystrophia Myotonica, DM) is another dynamic repeat disorder with a wide variability in the phenotype. DM has a progressive course, and both the age of onset and the course of the disease vary. These variables correlate partly with the trinucleotide repeat length [Harper, 2001]. As part of ongoing research in the departments of clinical genetics in Maastricht and Leuven, we focused on children and adolescents with the childhood onset type of DM. At this age, the subjects do not have significant muscle impairment [de Die-Smulders C., 2000], while families mention a diversity of mild cognitive and behavioural problems in these children. In an initial group containing 14 subjects with the childhood onset type, we demonstrated that these subjects have a general cognitive impairment: all the childhood onset subjects had an intelligence level below the average (Chapter 9), while this had previously only been described in subjects with the congenital type of DM.

In the two children with congenital type of DM included in the study, we found a cognitive decline over a two-year period. This was an incidental finding which should be studied further through longitudinal study in a larger group of congenital DM subjects. Confirmation of the latter finding would have consequences in the academic management of children with congenital DM.

Another finding was that an unexpected number of these children have social problems, and symptoms relating to Attention Deficit Hyperactivity Disorder (ADHD), and to Anxiety Disorder. This was confirmed in a subsequent study on 24 subjects with childhood onset DM, that were recruited with less or no referral bias and with the participation of the Dutch organisation for muscle disease patients (Chapter 10).

These findings on cognitive and behavioural problems in subjects with childhood onset DM who have no or minimal muscular impairment are additional evidence that DM is not only a progressive muscle disease but also a brain disorder, as has been proposed by others 
[Ashizawa, 1998]. Our hypothesis is that executive functions are most impaired. Evidence for this are the fact that ADHD in children reflects weakness of some executive functions, and the fact that apathy and even loss of decorum can be observed in DM subjects prior to significant muscle impairment [Harper, 2001]. The latter symptoms are generally associated with frontal lobe dysfunction. This hypothesis needs further research on the behavioural phenotype in DM, combined with functional brain imaging.

An important consequence of both studies is that clinicians should give the childhood type of DM the necessary attention. It should be differentiated from congenital DM in children, as the needs of children with childhood onset DM are in the domain of behaviour and academic performance, rather than in the domain of motor development. Another consequence of these studies is that the absence of significant muscle impairment is not sufficient to categorise children with a family risk for DM as not affected. Behavioural and/or cognitive complaints in children at risk should receive the necessary medical and psychological attention, as these may be early signs of the disease.

Clinical experience suggests that the course of childhood onset DM in the years after its onset may be different from that in congenital DM. While children with congenital DM catch up in several developmental areas in the first decades of life, the development of children with childhood onset seems to diverge more and more from an initially normal development. Longitudinal research on the behavioural phenotype is required in this and other types of DM. 
Part IV. ReFERENCES\& SumMaries 


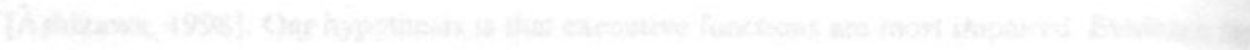

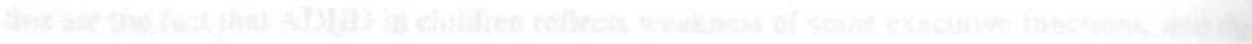

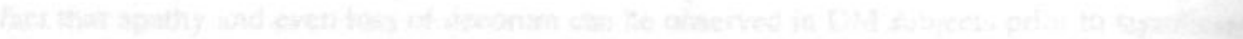

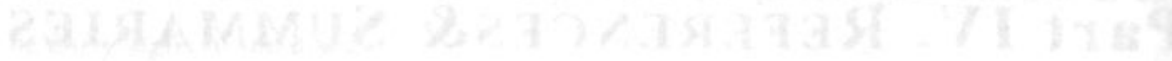




\section{References}

Abe K. Fujimura H, Toyooka K, Yorifuji S, Nishikawa Y, Hazama T, Yanagihara T. (1994). Involvement of the central nervous system in myotonic dystrophy. J Neurol Sci 127 (2):179. 185.

Abitbol M, Menini C, Delezoide AL, Rhyner T, Vekemans M, Mallet J. (1993). Nucleus basalis magnocellularis and hippocampus are the major sites of FMR-1 expression in the human fetal brain. Nat Genet 4 (2):147-153.

Abrams MT, Reiss AL, Freund LS, Baumgardner TL, Chase GA, Denckla MB. (1994). Molecularneurobehavioral associations in females with the fragile X full mutation. Am J Med Genet 51 (4):317-327.

Agulhon C, Blanchet P, Kobetz A, Marchant D, Faucon N, Sarda P, Moraine C, Sittler A, Biancalana V, Malafosse A, Abitbol M. (1999). Expression of FMR1, FXR1, and FXR2 genes in human prenatal tissues. $J$ Neuropathol Exp Neurol 58 (8):867-880.

Aicardi J, Conti D, Goutieres F. (1975). [Clinical and genetic aspects of the early form of Steinert's dystrophia myotonica]. J Genet Hum 23 SUPPL:146-157.

Allingham-Hawkins DJ, Brown CA, Babul R, Chitayat D, Krekewich K, Humphries T, Ray PN, Teshima IE. (1996). Tissue-specific methylation differences and cognitive function in fragile $\mathrm{X}$ premutation females. Am J Med Genet 64 (2):329-333.

Allingham-Hawkins DJ, Babul-Hirji R, Chitayat D, Holden JJ, Yang KT, Lee C, Hudson R, Gorwill H, Nolin SL, Glicksman A, Jenkins EC, Brown WT, Howard-Peebles PN, Becehi C, Cummings E, Fallon L, Seitz S, Black SH, Vianna-Morgante AM, Costa SS, Otto PA, Mingroni-Netto RC, Murray A, Webb J, MacSwinney F, Dennis N, Jacobs P, Syrrou M, Georgiou I, Patsalis PC, Giovannucci Uzielli ML, Guarducci S, Lapi E, Cecconi A, Ricci U, Ricotti G, Biondi C, Scarselli B, Vieri F. (1999). Fragile X premutation is a significant risk factor for premature ovarian failure: the International Collaborative POF in Fragile $\mathrm{X}$ study - . preliminary data. Am J Med Genet 83 (4):322-325.

Ambrosini PG, Nurnberg HG. (1979). Psychopathology: a primary feature of myotonic dystrophy. Psychosomatics 20 (6):393-395, 398-399.

American Psychiatric Association. (1987). Diagnostic and Statistical Manual of Mental Diseases. 3rd ed. Revised (DSM-III-R). Washington DC: American Psychiatric Association.

American Psychiatric Association. (1994). Diagnostic and Statistical Manual of Mental Disorders. IV. Washington D.C.: American Psychiatric Association.

Amir RE, Van den Veyver IB, Wan M, Tran CQ, Francke U, Zoghbi HY. (1999). Rett syndrome is caused by mutations in X-linked MECP2, encoding methyl- CpG-binding protein 2. Nat Genet $23(2): 185-188$.

Annane D, Fiorelli M, Mazoyer B, Pappata S, Eymard B, Radvanyi H, Junien C, Fardeau M, Merlet P, Gajdos P, Syrota A, Sansom Y, Duboc D. (1998). Impaired cerebral glucose metabolism in myotonic dystrophy: a triplet- size dependent phenomenon. Neuromuscul Disord 8 (1):39-45.

Ashizawa T. (1998). Myotonic dystrophy as a brain disorder. Arch Neurol 55 (3):291-293.

Ashley CT, Jr., Wilkinson KD, Reines D, Warren ST. (1993). FMR1 protein: conserved RNP family domains and selective RNA binding. Science 262 (5133):563-566.

Aslanidis C, Jansen G, Amemiya C, Shutler G, Mahadevan M, Tsilfidis C, Chen C, Alleman J, Wormskamp NG, Vooijs M, et al. (1992). Cloning of the essential myotonic dystrophy region and mapping of the putative defect. Nature 355 (6360):548-551.

Bachner D, Manca A, Steinbach P, Wohrle D, Just W, Vogel W, Hameister H, Poustka A. (1993). Enhanced expression of the murine FMR1 gene during germ cell proliferation suggests a special function in both the male and the female gonad. Hum Mol Genet 2 (12):2043-2050.

Bardoni B, Mandel J, Fisch G. (2000). FMR1 gene and fragile X syndrome. Am J Med Genet 97 (2): 153-163.

Bardoni B, Schenck A, Mandel JL. (2001). The Fragile X mental retardation protein. Brain Res Bull 56 (3-4):375-382.

Bassett AS, Hodgkinson K, Chow EW, Correia S, Scutt LE, Weksberg R. (1998). 22q11 deletion 
syndrome in adults with schizophrenia. Am J Med Genet 81 (4):328-337.

Beck AT, Ward CH, Mendelsohn M. Mock JE, Erbaugh J. (1961). An inventory for measuring depressions. Arch Gen Psychiatry 4:561-571.

Bennetto L, Pennington BF, Porter D, Taylor AK, Hagerman RJ. (2001). Profile of cognitive functioning in women with the fragile X mutation. Neuropsychology 15 (2):290-299.

Bird TD, Follett C. Griep E. (1983). Cognitive and personality function in myotonic muscular dystrophy. J Neurol Neurosurg Psychiatry 46 (11):971-980.

Blondis TA, Cook E, Koza-Taylor P, Finn T. (1996). Asperger syndrome associated with Steinert's myotonic dystrophy. Dev Med Child Neurol 9:840-847.

Boccia ML, Roberts JE. (2000). Behavior and autonomic nervous system function assessed via heart period measures: the case of hyperarousal in boys with fragile $\mathrm{X}$ syndrome. Behav Res Methods Insirum Comput 32 (1):5-10.

Bolton P. Macdonald H, Pickles A, Rios P, Goode S, Crowson M, Bailey A, Rutter M. (1994). A casecontrol family history study of autism. J Child Psychol Psychiatry 35 (5):877-900.

Bolton PF, Griffiths PD. (1997). Association of tuberous sclerosis of temporal lobes with autism and atypical autism. Lancet 349 (9049):392-395.

Borghgraef M. Fryns JP, Dielkens A, Pyck K, Van den Berghe H. (1987). Fragile (X) syndrome: a study of the psychological profile in 23 prepubertal patients. Clin Genet 32 (3):179-186.

Borghgraef M (1991): Psychological Profiles and Behavioural Characteristics in Chromosomal Syndromes. [Doctoral thesis]. University of Maastricht.

Borghgraef M. Swillen A, Van den Berghe H, Fryns JP. (1995). Fragile X boys: evolution of the mental age in childhood. Preliminary data on 10 prepubertal boys. Genet Couns 6 (2):97-101.

Bouman K, Luteijn F, Albersnagel FA. van der Ploeg FAE. (1985). Enige ervaringen met de Beck Depression Inventory. Tijdsch Psychol 13:13-24.

Brainard SS, Schreiner RA. Hagerman RJ. (1991). Cognitive profiles of the carrier fragile X woman. Am J Med Genet 38 (2-3):505-508.

Brandt J, Bylsma FW, Gross R, Stine OC, Ranen N, Ross CA. (1996). Trinucleotide repeat length and clinical progression in Huntington's disease. Neurology 46 (2):527-531.

Braun K. Segal M. (2000). FMRP involvement in formation of synapses among cultured hippocampal neurons. Cereb Cortex 10 (10); 1045-1052.

Brook JD, McCurrach ME, Harley HG, Buckler AJ, Church D, Aburatani H, Hunter K, Stanton VP, Thirion JP, Hudson T, et al. (1992). Molecular basis of myotonic dystrophy: expansion of a trinucleotide (CTG) repeat at the $3^{\prime}$ end of a transcript encoding a protein kinase family member. Cell 68 (4):799-808.

Broughton R, Stuss D, Kates M, Roberts J, Dunham W. (1990). Neuropsychological deficits and sleep in myotonic dystrophy. Can J Neurol Sci 17 (4):410-415.

Brown WT, Jenkins EC, Friedman E, Brooks J, Wisniewski K, Raguthu S, French J. (1982). Autism is associated with the fragile-X syndrome. J Autism Dev Disord 12 (3):303-308.

Brumback RA, Carlson KM. (1983). The depression of myotonic dystrophy: response to imipramine. $J$ Neurol Neurosurg Psychiairy 46 (6):587-588.

Brunner HG, Nelen M, Breakefield XO. Ropers HH, van Oost BA. (1993). Abnormal behavior associated with a point mutation in the structural gene for monoamine oxidase A. Science 262 (5133):578-580.

Brunner HG. (1996). MAOA deficiency and abnormal behaviour: perspectives on an association. Ciba Found Symp 194:155-164.

Bungener C. Jouvent R, Delaporte C. (1998). Psychopathological and emotional deficits in myotonic dystrophy. J Neurol Neurosurg Psychiatry 65 (3):353-356.

Buxton J, Shelbourne P, Davies J, Jones C, Van Tongeren T, Aslanidis C, de Jong P, Jansen G, Anvret M, Riley B, et al. (1992). Detection of an unstable fragment of DNA specific to individuals with myotonic dystrophy. Nature 355 (6360):547-548.

Calderon R. (1966). Myotonic dystrophy: a neglected cause of mental retardation. $J$ Pediatr 68 (3):423-431.

Carrie A, Jun L, Bienvenu T, Vinet MC, McDonell N, Couvert P, Zemni R, Cardona A, Van Buggenhout G, Frints S, Hamel B, Moraine C, Ropers HH, Strom T, Howell GR, Whittaker A. Ross MT, Kahn A, Fryns JP. Beldjord C, Marynen P. Chelly J. (1999). A new member of 
the IL-1 receptor family highly expressed in hippocampus and involved in X-linked mental retardation. Nat Genet 23 (1):25-31.

Célistin-Westreich S. Ponjaert 1. (1998). Adaptation of the "Amsterdams Diagnostisch Interview voor Kinderen en Adolescenten" (ADIKA) to the DSM-IV. Internal Report. Brussel: Vrije Universiteit Brussel.

Censori B. Danni M. Del Pesce M. Provinciali L. (1990). Neuropsychological profile in myotonic dystrophy. $J$ Neurol 237 (4):251-256.

Censori B. Provinciali L. Danni M. Chiaramoni L. Maricotti M. Foschi N. Del Pesce M. Salvolini U. (1994). Brain involvement in myotonic dystrophy: MRI features and their relationship to clinical and cognitive conditions. Acta Neurol Scand 90 (3):211-217.

Chang L. Anderson T, Migneco OA, Boone K, Mehringer CM, Villanueva-Meyer J, Berman N, Mena L. (1993). Cerebral abnormalities in myotonic dystrophy. Cerebral blood flow, magnetic resonance imaging, and neuropsychological tests. Arch Neurol 50 (9):917-923.

Chang L, Ernst T, Osborn D, Seltzer W, Leonido-Yee M, Poland RE. (1998). Proton spectroscopy in myotonic dystrophy: correlations with CTG repeats. Arch Neurol 55 (3):305-311.

Chelly J. Mandel JL. (2001). Monogenic causes of X-linked mental retardation. Nat Rev Genet 2 (9):669-680.

Chen L. Toth M. (2001). Fragile X mice develop sensory hyperreactivity to auditory stimuli. Neuroscience 103 (4): 1043-1050.

Chiurazzi P (2001a): Origin and reactivation of the fragile $X$ gene. [Doctoral thesis]. Erasmus Universiteit Rotterdam.

Chiurazzi P. (2001b) On-line XLMR database. World Wide Web: http://xlmr interfree ithome. $h t m$.

Chiurazzi P. Hamel BC, Neri G. (2001). XLMR genes: update 2000. Eur J Hum Genet 9 (2):71-81.

Cianchetti C, Filippi G, Sannio-Fancello G, Fratta AL, Marrosu MG, Dagna-Bricarelli F, Siniscalco M. (1992). Premutation for the Martin-Bell syndrome analyzed in a large Sardinian family: II. Neuropsychological and behavioral data. Am J Med Genet 43 (1-2): 103-110.

Cicchetti D, Cohen DJ. (1995). Developmental Psychopathology, Vol. I. New York: John Wiley \& Sons.

Claes S, Devriendt K, Lagae L, Ceulemans B, Dom L, Casaer P, Raeymaekers P, Cassiman JJ, Fryns JP. (1997). The X-linked infantile spasms syndrome (MIM 308350) maps to Xp11.4- Xpter in two pedigrees. Ann Neurol 42 (3):360-364.

Claustres M, Guittard C, Bozon D, Chevalier F, Verlingue C, Ferec C, Girodon E, Cazeneuve C, Bienvenu T, Lalau G, Dumur V, Feldmann D, Bieth E, Blayau M, Clavel C, Creveaux I, Malinge MC, Monnier N, Malzac P, Mittre H, Chomel JC, Bonnefont JP, Iron A, Chery M, Georges MD. (2000). Spectrum of CFTR mutations in cystic fibrosis and in congenital absence of the vas deferens in France. Hum Mutat 16 (2):143-156.

Colombo G, Perini GI, Miotti MV, Armani M, Angelini C. (1992). Cognitive and psychiatric evaluation of 40 patients with myotonic dystrophy. Ital J Neurol Sci 13 (1):53-58.

Comings DE, Wu S, Chiu C, Ring RH, Gade R, Ahn C, MacMurray JP, Dietz G, Muhleman D. (1996). Polygenic inheritance of Tourette syndrome, stuttering, attention deficit hyperactivity, conduct, and oppositional defiant disorder: the additive and subtractive effect of the three dopaminergic genes--DRD2, D beta H, and DAT1. Am J Med Genet 67 (3):264-288.

Comings DE, Gade-Andavolu R, Gonzalez N, Blake H, Wu S, MacMurray JP. (1999). Additive effect of three noradrenergic genes (ADRA2a, ADRA2C, DBH) on attention-deficit hyperactivity disorder and learning disabilities in Tourette syndrome subjects. Clin Genet 55 (3):160-172.

Comings DE, Gade-Andavolu R, Gonzalez N, Wu S, Muhleman D, Blake H, Dietz G, Saucier G, MacMurray JP. (2000). Comparison of the role of dopamine, serotonin, and noradrenaline genes in ADHD, ODD and conduct disorder: multivariate regression analysis of 20 genes. Clin Genet 57 (3):178-196.

Cornish KM, Munir F, Cross G. (1999). Spatial cognition in males with Fragile-X syndrome: evidence for a neuropsychological phenotype. Corlex 35 (2):263-271.

Cruts M, Van Broeckhoven C. (1998). Molecular genetics of Alzheimer's disease. Ann Med 30 (6):560-565.

Curfs LM, Borghgraef M, Wiegers A, Schreppers-Tijdink GA, Fryns JP. (1989). Strengths and weaknesses in the cognitive profile of fra(X) patients. Clin Genet $36(6): 405-410$. 
Cuthill J. Gattereau A. Viguie F. (1988). Myotonic dystrophy of Steinert: are anxiety and depression necessarily concomitants? Can J Psychiatry 33 (3):203-206.

Daker MG. (1983). Fragile X chromosome in normal males [letter]. Am J Med Genet 14 (4):795-797.

Damian MS, Bachmann G, Koch MC, Schilling G, Stoppler S, Dorndorf W. (1994a). Brain disease and molecular analysis in myotonic dystrophy. Neuroreport 5 (18):2549-2552.

Damian MS, Schilling G, Bachmann G, Simon C, Stoppler S, Dorndorf W. (1994b). White matter lesions and cognitive deficits: relevance of lesion pattern? Acta Neurol Scand 90 (6):430-436.

de Die-Smulders C (2000): Long-term clinical and genetic studies in myotonic dystrophy. [Doctoral Thesis]. Universiteit Maastricht.

de Die-Smulders CE, Höweler CJ, Thijs C, Mirandolle JF, Anten HB, Smeets HJ, Chandler KE, Geraedts JP. (1998). Age and causes of death in adult-onset myotonic dystrophy. Brain 121 (Pt 8):1557-1563.

de Sonneville L, Njiokiktjien C. (1988). Aspects of information processing., Vol. 2. Amsterdam: SUYI.

de Vries BB, Wiegers AM, Smits AP, Mohkamsing S, Duivenvoorden HJ, Fryns JP, Curfs LM, Halley DJ, Oostra BA, van den Ouweland AM, Niermeijer MF. (1996). Mental status of females with an FMRI gene full mutation. Am J Hum Genet 58 (5): 1025-1032.

de Vries BB. Mohkamsing S, van den Ouweland AM, Halley DJ. Niermeijer MF, Oostra BA, Willemsen R. (1998). Screening with the FMRI protein test among mentally retarded males. Hum Genel 103 (4):520-522.

Delaporte C. (1998). Personality patterns in patients with myotonic dystrophy. Arch Neurol 55 (5):635-640

Di Costanzo A. Di Salle F, Santoro L. Bonavita V, Tedeschi G. (2001). T2 relaxometry of brain in myotonic dystrophy. Neuroradiology 43 (3): 198-204.

Dobkin CS, Nolin SL, Cohen I, Sudhalter V, Bialer MG, Ding XH, Jenkins EC, Zhong N, Brown WT. (1996). Tissue differences in fragile $\mathrm{X}$ mosaics: mosaicism in blood cells may differ greatly from skin. Am J Med Genet 64 (2):296-301.

Duveneck MJ, Portwood MM. Wicks JJ, Lieberman JS. (1986). Depression in myotonic muscular dystrophy. Arch Phys Med Rehabil 67 (12):875-877.

Duyao M, Ambrose C, Myers R, Novelletto A, Persichetti F, Frontali M, Folstein S, Ross C, Franz M, Abbott M, et al. (1993). Trinucleotide repeat length instability and age of onset in Huntington's disease. Nat Genet 4 (4):387-392.

Dyer-Friedman J, Glaser B, HessI D, Johnston C, Huffman LC, Taylor A, Wisbeck J, Reiss AL. (2002). Genetic and environmental influences on the cognitive outcomes of children with fragile X syndrome. J Am Acad Child Adolesc Psychiatry 41 (3):237-244.

Dykens EM. (1995). Measuring behavioral phenotypes: provocations from the "new genetics". Am J Ment Retard 99 (5):522-532.

Einfeld SL. Tonge BJ. (1996). Population prevalence of psychopathology in children and adolescents with intellectual disability: II. Epidemiological findings. J Intellect Disabil Res 40 (2):99-109.

Evans KL, Muir WJ, Blackwood DH, Porteous DJ. (2001). Nuts and bolts of psychiatric genetics: building on the Human Genome Project. Trends Genet 17 (1):35-40.

Faraone SV, Biederman J. (1998). Neurobiology of attention-deficit hyperactivity disorder. Biol Psychiatry 44 (10):951-958.

Faulkner CL, Kingston HM. (1998). Knowledge, views, and experience of 25 women with myotonic dystrophy. J Med Genet 35 (12): 1020-1025.

Feng Y, Zhang F, Lokey LK, Chastain JL, Lakkis L, Eberhart D, Warren ST. (1995). Translational suppression by trinucleotide repeat expansion at FMR1. Science 268 (5211):731-734.

Fiorelli M, Duboc D, Mazoyer BM, Blin J, Eymard B, Fardeau M, Samson Y. (1992). Decreased cerebral glucose utilization in myotonic dystrophy. Neurology 42 (1):91-94.

Fisch GS. (1992). Is autism associated with the fragile X syndrome? Am J Med Genet 43 (1-2):47-55.

Fisch GS, Nelson DL, Snow K. Thibodeau SN, Chalifoux M, Holden JJ. (1994). Reliability of diagnostic assessment of normal and premutation status in the fragile $\mathrm{X}$ syndrome using DNA testing. Am J Med Genet 51 (4):339-345.

Fisch GS, Snow K. Thibodeau SN, Chalifaux M. Holden JJ, Nelson DL, Howard-Peebles PN, Maddalena A. (1995). The fragile X premutation in carriers and its effect on mutation size in 
offspring. Am $J$ Hum Genet 56 (5):1147-1155.

Fisch GS, Simensen R. Tarleton J. Chalifoux M, Holden J., Carpenter N, Howard-Peebles PN, Maddalena A. (1996). Longitudinal study of cognitive abilities and adaptive behavior levels in fragile X males: a prospective multicenter analysis. Am J Med Genet 64 (2):356-361.

Fisch GS, Carpenter N, Holden JJ. Howard-Peebles PN, Maddalena A. Borghgraef M. Steyaert J. Fryns JP. (1999a). Longitudinal changes in cognitive and adaptive behavior in fragile $\mathrm{X}$ females: a prospective multicenter analysis. Am J Med Genet 83 (4):308-312.

Fisch GS, Holden JJ, Carpenter NJ, Howard-Peebles PN, Maddalena A. Pandya A. Nance W. (1999b). Age-related language characteristics of children and adolescents with fragile $\mathrm{X}$ syndrome. Am $J$ Med Genet 83 (4):253-256.

Fisch GS. (2000). Psychological assessment in XLMR: a proposal for setting international standards. Genet Couns II (2):85-101.

Flint J, Yule W: Behavioural phenotypes. (1994). In: Rutter M, Taylor E, Hersov LA, eds. Child and adolescent psychiatry: modern approaches. Oxford: Blackwell Scientific.

Flint J. (1998). Behavioral phenotypes: conceptual and methodological issues. Am J Med Genet 81 (3):235-240.

Fowler WM, Jr., Abresch RT, Koch TR, Brewer ML, Bowden RK, Wanlass RL. (1997). Employment profiles in neuromuscular diseases. Am J Phys Med Rehabil 76 (1):26-37.

Franke P, Maier W, Hautzinger M, Weiffenbach O, Gansicke M, Iwers B, Poustka F, Schwab SG, Froster U. (1996). Fragile-X carrier females: evidence for a distinct psychopathological phenotype? Am J Med Genet 64 (2):334-339.

Franke P, Leboyer M, Gansicke M. Weiffenbach O. Biancalana V, Cornillet-Lefebre P, Croquette MF, Froster U, Schwab SG, Poustka F, Hautzinger M, Maier W. (1998). Genotype-phenotype relationship in female carriers of the premutation and full mutation of FMR-1. Psychiatry Res 80 (2):113-127.

Franke P, Leboyer M, Hardt J, Sohne E, Weiffenbach O, Biancalana V, Cornillet-Lefebre P, Delobel B, Froster U, Schwab SG, Poustka F, Hautzinger M, Maier W. (1999). Neuropsychological profiles of FMR-1 premutation and full-mutation carrier females. Psychiatry Res 87 (2-3):223231.

Franzese A, Antonini G, Iannelli M, Leardi MG, Spada S, Vichi R, Millefiorini M, Lazzari R. (1991). Intellectual functions and personality in subjects with noncongenital myotonic muscular dystrophy. Psychol Rep 68 (3):723-732.

Freund LS, Reiss AL, Abrams MT. (1993). Psychiatric disorders associated with fragile X in the young female. Pediatrics 91 (2):321-329.

Frints S, Froyen G, Marynen P, Fryns J-P. (2002). Mini-review: X-linked mental retardation (XLMR): Vanishing boundaries between nonspecific (MRX) and syndromic (MRXS) forms. Clin Genet In press

Fryns JP. (1984). The fragile X syndrome. A study of 83 families. Clin Genet 26 (6):497-528.

Fryns JP, Jacobs J, Kleczkowska A, van den Berghe H. (1984a). The psychological profile of the fragile X syndrome. Clin Genet 25 (2):131-134.

Fryns JP, Kleczkowska A, Kubien E, Petit P, Van den Berghe H. (1984b). Inactivation pattern of the fragile X in heterozygous carriers. Hum Genet 65 (4):400-401.

Fryns JP. (1986). The female and the fragile X. A study of 144 obligate female carriers. Am J Med Genet 23 (1-2):157-169.

Fryns JP: X-linked mental retardation and the Fragile X syndrome: a clinical approach. (1989). In: Davis KE, ed. The Fragile X syndrome. Oxford, New York, Tokyo: Oxford University Press, 1-39.

Fu YH, Kuhl DP, Pizzuti A, Pieretti M, Sutcliffe JS, Richards S, Verkerk AJ, Holden JJ, Fenwick RG, Jr., Warren ST, et al. (1991). Variation of the CGG repeat at the fragile X site results in genetic instability: resolution of the Sherman paradox. Cell 67 (6): 1047-1058.

Gantois I, Kooy RF. (2002). Targeting fragile X. Genome Biol 3 (5):1014.1011-1014.1015.

Gantois I, Reyniers E, Kooy F. (2002). Proceedings of the 10th International Workshop on Fragile X and XLMR: Identification of genes differentially expressed in the fragile X knockout mouse. Genet Couns 13 (2):207-261.

Gelernter J: Clinical molecular genetics. (1999). In: Charney DS, Nestler EJ, Bunney BS, eds. 
Neurobiology of mental illness. New York: Oxford University Press, 108-120.

Genc B, Muller-Hartmann H. Zeschnigk M, Deissler H, Schmitz B, Majewski F, von Gontard A, Doerfler W. (2000). Methylation mosaicism of $5^{\prime}-(\mathrm{CGG})(\mathrm{n})-3^{\prime}$ repeats in fragile $\mathrm{X}$, premutation and normal individuals. Nucleic Acids Res 28 (10):2141-2152.

Gillberg C, Wahlstrom J. (1985). Chromosome abnormalities in infantile autism and other childhood psychoses: a population study of 66 cases. Dev Med Child Neurol 27 (3):293-304.

Gillberg C. (1998). Chromosomal disorders and autism. J Autism Dev Disord 28 (5):415-425.

Gilmartin JJ, Cooper BG, Griffiths CJ. Walls TJ, Veale D, Stone TN, Osselton JW, Hudgson P, Gibson GJ. (1991). Breathing during sleep in patients with myotonic dystrophy and nonmyotonic respiratory muscle weakness. QJ Med 78 (285):21-31.

Giubilei F, Antonini G, Bastianello S, Morino S, Paolillo A, Fiorelli M, Ferretti C, Fieschi C. (1999). Excessive daytime sleepiness in myotonic dystrophy. J Neurol Sci 164 (1):60-63.

Glantz RH, Wright RB, Huckman MS, Garron DC, Siegel IM. (1988). Central nervous system magnetic resonance imaging findings in myotonic dystrophy. Arch Neurol 45 (1):36-37.

Goossens E, Steyaert J, De Die-Smulders C, Willekens D, Fryns JP. (2000). Emotional and behavioral profile and child psychiatric diagnosis in the childhood type of myotonic dystrophy. Genet Couns II (4):317-327.

Gratacos M, Nadal M, Martin-Santos R, Pujana MA, Gago J, Peral B, Armengol L, Ponsa I, Miro R, Bulbena A. Estivill X. (2001). A polymorphic genomic duplication on human chromosome 15 is a susceptibility factor for panic and phobic disorders. Cell 106 (3):367-379.

Gusella JF. (1984). Genetic linkage of the Huntington's disease gene to a DNA marker. Can J Neurol Sci 11 (4):421-425.

Guttler F, Guldberg P. (2000). Mutation analysis anticipates dietary requirements in phenylketonuria. Eur J Pediatr 159 Suppl 2:S150-153.

Hageman AT, Gabreels FJ, Liem KD, Renkawek K, Boon JM. (1993). Congenital myotonic dystrophy; a report on thirteen cases and a review of the literature. $J$ Neurol Sci 115 (1):95101.

Hagerman R, Kemper M, Hudson M. (1985). Learning disabilities and attentional problems in boys with the fragile X syndrome. Am J Dis Child 139 (7):674-678.

Hagerman RJ, McBogg P. Hagerman PJ. (1983). The fragile X syndrome: history, diagnosis, and treatment. J Dev Behav Pediatr 4 (2):122-130.

Hagerman RJ, Chudley AE, Knoll JH, Jackson AWd, Kemper M, Ahmad R. (1986a). Autism in fragile X females. Am J Med Genet 23 (1-2):375-380.

Hagerman RJ, Jackson AWd, Levitas A, Rimland B, Braden M. (1986b). An analysis of autism in fifty males with the fragile $\mathrm{X}$ syndrome. Am J Med Genet 23 (1-2):359-374.

Hagerman RJ, Murphy MA, Wittenberger MD. (1988). A controlled trial of stimulant medication in children with the fragile X syndrome. Am J Med Genet 30 (1-2):377-392.

Hagerman RJ, Schreiner RA, Kemper MB, Wittenberger MD, Zahn B, Habicht K. (1989). Longitudinal IQ changes in fragile X males. Am J Med Genet 33 (4):513-518.

Hagerman RJ, Jackson C, Amiri K, Silverman AC, OConnor R, Sobesky W. (1992). Girls with fragile X syndrome: physical and neurocognitive status and outcome. Pediatrics 89 (3):395-400,

Hagerman RJ. Hull CE, Safanda JF, Carpenter I, Staley LW, OConnor RA, Seydel C, Mazzocco MM. Snow K. Thibodeau SN, et al. (1994). High functioning fragile X males: demonstration of an unmethylated fully expanded FMR-1 mutation associated with protein expression. Am J Med Genet 51 (4):298-308.

Hagerman RJ, Staley LW, OConner R, Lugenbeel K, Nelson D, McLean SD, Taylor A. (1996). Learning-disabled males with a fragile X CGG expansion in the upper premutation size range. Pediatrics 97 (1):122-126.

Hagerman RJ, Hills J, Scharfenaker S, Lewis H. (1999). Fragile X syndrome and selective mutism. Am J Med Genet 83 (4):313-317.

Hansen RS, Gartler SM, Scott CR, Chen SH, Laird CD. (1992). Methylation analysis of CGG sites in the CpG island of the human FMRI gene. Hum Mol Genet 1 (8):571-578.

Hanson DM, Jackson AWd, Hagerman RJ. (1986). Speech disturbances (cluttering) in mildly impaired males with the Martin-Bell/fragile X syndrome. Am J Med Genet 23 (1-2):195-206.

Harley HG, Brook JD, Rundle SA, Crow S, Reardon W, Buckler AJ, Harper PS, Housman DE, Shaw 
DJ. (1992a). Expansion of an unstable DNA region and phenotypic variation in myotonic dystrophy. Nature 355 (6360):545-546.

Harley HG, Rundle SA. Reardon W, Myring J, Crow S, Brook JD, Harper PS, Shaw DJ. (1992b). Unstable DNA sequence in myotonic dystrophy. Lancet 339 (8802):1125-1128.

Harley HG, Rundle SA, McMillan JC, Myring J, Brook JD, Crow S, Reardon W. Fenton L. Shaw DJ, Harper PS. (1993). Size of the unstable CTG repeat sequence in relation to phenotype and parental transmission in myotonic dystrophy. Am J Hum Genet 52:1164-1174.

Harper PS. (1975). Congenital myotonic dystrophy in Britain. 1. Clinical aspects. Arch Dis Child 50 (7):505-513.

Harper PS, Harley HG, Reardon W, Shaw DJ. (1992). Anticipation in myotonic dystrophy: new light on an old problem. Am J Hum Genet 51 (1):10-16.

Harper PS: Myotonic Dystrophy as a trinucleotide repeat disorder - a clinical perspective. (1998). In: Wells RD, Waren ST, eds. Hereditary Neurological Diseases. San Diego: Academic press.

Harper PS. (2001). Myotonic dystrohpy, Vol. 37. London: W.B. Saunders.

Harris JC. (1998a). Developmental Neuropsychiatry, Vol. II. New York: Oxford University Press.

Harris JC. (1998b). Developmental Neuropsychiatry, Vol. I. New York: Oxford University Press.

Hashimoto T. Tayama M. Miyazaki M, Murakawa K, Kawai H. Nishitani H, Kuroda Y. (1995). Neuroimaging study of myotonic dystrophy. L. Magnetic resonance imaging of the brain. Brain Dev 17 (1):24-27.

Heitz D, Devys D. Imbert G, Kretz C, Mandel JL. (1992). Inheritance of the fragile X syndrome: size of the fragile $\mathrm{X}$ premutation is a major determinant of the transition to full mutation. $J$ Med Genet 29 (11):794-801.

Higgins JJ, Rosen DR, Loveless JM, Clyman JC, Grau MJ. (2000). A gene for nonsyndromic mental retardation maps to chromosome 3p25-pter. Neurology 55 (3):335-340.

Homer. (1995). Odyssey, books 1-12, Vol. 1: Harvard College.

Howard-Peebles PN, Stoddard GR, Mims MG. (1979). Familial X-linked mental retardation, verbal disability, and marker X chromosomes. Am J Hum Genet 31 (2):214-222.

Howard-Peebles PN. (1982). Non-specific X-linked mental retardation: background, types, diagnosis and prevalence. J Ment Defic Res 26 (4):205-213.

Höweler CJ, Busch HF, Geraedts JP, Niermeijer MF, Staal A. (1989). Anticipation in myotonic dystrophy: fact or fiction? Brain 112 (3):779-797.

Huber SJ, Kissel JT, Shuttleworth EC, Chakeres DW, Clapp LE, Brogan MA. (1989). Magnetic resonance imaging and clinical correlates of intellectual impairment in myotonic dystrophy. Arch Neurol 46 (5):536-540.

Hull C, Hagerman RJ. (1993). A study of the physical, behavioral, and medical phenotype, including anthropometric measures, of females with fragile X syndrome. Am J Dis Child 147 (11):12361241.

Hunt A, Shepherd C. (1993). A prevalence study of autism in tuberous sclerosis. J Autism Dev Disord $23(2): 323-339$.

Hunter A, Tsilfidis C, Mettler G, Jacob P, Mahadevan M, Surh L, Korneluk R. (1992). The correlation of age of onset with CTG trinucleotide repeat amplification in myotonic dystrophy. $J$ Med Genet 29 (11):774-779.

Hurst JA, Baraitser M, Auger M, Graham F, Norell S. (1990). An extended family with a dominantly inherited speech disorder. Dev Med Child Neurol 32:347-355.

Irvine RA, Yu MC, Ross RK, Coetzee GA. (1995). The CAG and GGC microsatellites of the androgen receptor gene are in linkage disequilibrium in men with prostate cancer. Cancer Res 55 (9):1937-1940.

ISPG. (2002) International Society for Psychiatric Genetics. World Wide Web: http://www.ispg.net/.

Jamain S, Betancur C, Quach H, Philippe A, Fellous M, Giros B, Gillberg C, Leboyer M, Bourgeron T. (2002). Linkage and association of the glutamate receptor 6 gene with autism. Mol Psychiatry 7 (3):302-310.

Jin P, Warren ST. (2000). Understanding the molecular basis of fragile X syndrome. Hum Mol Genet 9 (6):901-908.

Johnson ER, Abresch RT, Carter GT, Kilmer DD, Fowler WM, Jr., Sigford BJ, Wanlass RL. (1995). Profiles of neuromuscular diseases. Myotonic dystrophy. Am J Phys Med Rehabil 74 (5 
Suppl):S104-116.

Johnson GE. (1897). Contribution to the psychology and pedagogy of feeble-minded children. Journal of psycho-asthenics 2:26-32.

Johnson K, Siciliano G. (1995). Report on MDA workshop on myotonic dystrophy, 10 October 1994, Montreal, Quebec, Canada. J Med Genet 32:662-665.

Johnston C, Eliez S, Dyer-Friedman J, Hessl D, Glaser B, Blasey C, Taylor A, Reiss A. (2001). Neurobehavioral phenotype in carriers of the fragile X premutation. Am J Med Genet 103 (4):314-319.

Kates WR, Abrams MT, Kaufmann WE, Breiter SN, Reiss AL. (1997). Reliability and validity of MRI measurement of the amygdala and hippocampus in children with fragile $\mathrm{X}$ syndrome. Psychiatry Res 75 (1):31-48.

Katsanis N, Lupski JR, Beales PL. (2001). Exploring the molecular basis of Bardet-Biedl syndrome. Hum Mol Genel 10 (20):2293-2299.

Kayl AE, Moore BD, 3rd. (2000). Behavioral phenotype of neurofibromatosis, type 1. Ment Retard Dev Disabil Res Rev 6 (2):117-124.

Kemper MB, Hagerman RJ, Ahmad RS, Mariner R. (1986). Cognitive profiles and the spectrum of clinical manifestations in heterozygous fra (X) females. Am J Med Genet 23 (1-2):139-156.

Kendler KS. (1996). Models for the joint effect of genotype and environment on liability to psychiatric illness. Am J Psychiatry 143:279-289.

Kenneson A, Zhang F, Hagedorn CH, Warren ST. (2001). Reduced FMRP and increased FMR1 transcription is proportionally associated with CGG repeat number in intermediate-length and premutation carriers. Hum Mol Genet 10 (14): 1449-1454.

Kimura T, Takahashi MP, Okuda Y, Kaido M, Fujimura H, Yanagihara T, Sakoda S. (2000). The expression of ion channel mRNAs in skeletal muscles from patients with myotonic muscular dystrophy. Neurosci Letl 295 (3):93-96.

Klesert TR, Cho DH, Clark JI, Maylie J, Adelman J, Snider L, Yuen EC, Soriano P, Tapscott SJ. (2000). Mice deficient in Six5 develop cataracts: implications for myotonic dystrophy. Nat Genet 25 (1): 105-109.

Kohen D, Burgess AP, Catalan J, Lant A. (1998). The role of anxiety and depression in quality of life and symptom reporting in people with diabetes mellitus. Qual Life Res 7 (3):197-204.

Kolb B, Whishaw I: Fundamentals of Human Neuropsychology. (1996)., Fourth edition ed. New York: W.H. Freeman and Company, 305-333.

Korenberg JR, Kurnit DM: Molecular and stochastic basis of congenital heart disease in Down syndrome. (1995). In: Clark EB, Markwald RR, Takao A, eds. Developmental mechanisms of heart disease. New York: Futura Publishing Co., Inc., 581-596.

Kortenbout van der Sluijs MJ, De Levita DJ, De Manen R, Defares BP. (1993). Amsterdams Diagnostisch Interview voor Kinderen en Adolescenten. Lisse (NI): Swets \& Zeitlinger.

Kwon H, Menon V, Eliez S, Warsofsky IS, White CD, Dyer-Friedman J, Taylor AK, Glover GH, Reiss AL. (2001). Functional neuroanatomy of visuospatial working memory in fragile $\mathrm{X}$ syndrome: relation to behavioral and molecular measures. Am J Psychiatry 158 (7):10401051.

La Spada AR, Wilson EM, Lubahn DB, Harding AE, Fischbeck KH. (1991). Androgen receptor gene mutations in X-linked spinal and bulbar atrophy. Nature 352:77-79.

Laberge C. (1989). Myotonic dystrophy in Quebec: geographical distribution and concept of genetic homogeneity. Can J Neurol Sci 16 (1):123-128.

Lai SL, Fisher S, Hurst JA, Vargha-Khadems F, Monaco AP. (2001). A forkhead-domain gene is mutated in a severe speech and language disorder. Nature 413:519-523.

Lavedan C, Hofmann-Radvanyi H, Shelbourne P, Rabes JP, Duros C, Savoy D, Dehaupas I, Luce S, Johnson K, Junien C. (1993). Myotonic dystrophy: size- and sex-dependent dynamics of CTG meiotic instability, and somatic mosaicism. Am J Hum Genet 52 (5):875-883.

Laxova R. (1994). Fragile X syndrome. Adv Pediatr 41:305-342.

Le Couteur A, Bailey A, Goode S, Pickles A, Robertson S, Gottesman I, Rutter M. (1996). A broader phenotype of autism: the clinical spectrum in twins. J Child Psychol Psychiatry 37 (7):785801.

Lehrke RG. (1974). X-linked mental retardation and verbal disability. Birth Defects Orig Artic Ser 10 
(1): $1-100$.

Li Z, Zhang Y, Ku L, Wilkinson KD, Warren ST, Feng Y. (2001). The fragile X mental retardation protein inhibits translation via interacting with mRNA. Nucleic Acids Res 29 (11):2276-2283.

Liquori CL, Ricker K, Moseley ML, Jacobsen JF, Kress W, Naylor SL, Day JW, Ranum LP. (2001). Myotonic dystrophy type 2 caused by a CCTG expansion in intron 1 of ZNF9. Science 293 (5531):864-867.

Loesch DZ. Hay DA, Mulley J. (1994). Transmitting males and carrier females in fragile X-revisited. Am J Med Genet 51 (4):392-399.

Lovell RW, Reiss AL. (1993). Dual diagnoses. Psychiatric disorders in developmental disabilities. Pediatr Clin North Am 40 (3):579-592.

Lubs HA. (1969). A marker X chromosome. Am J Hum Genet 21 (3):231-244.

Luteijn F. (1981). Kinder depressieschaal (Nederlandstalige bewerking van de Children's Depression Scale). Lisse: Swets \& Zeitlinger.

Maes B, Fryns JP, Van Walleghem M, Van den Berghe H. (1993). Fragile-X syndrome and autism: a prevalent association or a misinterpreted connection? Genet Couns 4 (4):245-263.

Maes B, Fryns JP, Van Walleghem M, Van den Berghe H. (1994). Cognitive functioning and information processing of adult mentally retarded men with fragile-X syndrome. Am J Med Genet 50 (2):190-200.

Malter HE, Iber JC, Willemsen R, de Graaff E, Tarleton JC, Leisti J, Warren ST, Oostra BA. (1997). Characterization of the full fragile $\mathrm{X}$ syndrome mutation in fetal gametes. Nat Genet is (2):165-169.

Manning JT, Baron-Cohen S, Wheelwright S, Sanders G. (2001). The 2nd to 4th digit ratio and autism. Dev Med Child Neurol 43 (3): 160-164.

Marozzi A, Vegetti W, Manfredini E, Tibiletti MG, Testa G, Crosignani PG, Ginelli E, Meneveri R, Dalpra L. (2000). Association between idiopathic premature ovarian failure and fragile $\mathrm{X}$ premutation. Hum Reprod 15 (1): 197-202.

Martin JP, Bell J. (1943). A pedigree of mental defect showing sex linkage. Journal of Neurological Psychiairy 6:154-157.

Martorell L, Monckton DG, Gamez J, Johnson KJ, Gich I, de Munain AL, Baiget M. (1998). Progression of somatic CTG repeat length heterogeneity in the blood cells of myotonic dystrophy patients. Hum Mol Genet 7 (2):307-312.

Mathieu J, Allard P, Potvin L, Prevost C, Begin P. (1999). A 10-year study of mortality in a cohort of patients with myotonic dystrophy. Neurology 52 (8):1658-1662.

Mazzocco MM, Hagerman RJ, Cronister-Silverman A, Pennington BF. (1992). Specific frontal lobe deficits among women with the fragile $\mathrm{X}$ gene. J Am Acad Child Adolesc Psychiatry 31 (6): 1141-1148.

Mazzocco MM, Holden JJ. (1996). Neuropsychological profiles of three sisters homozygous for the fragile X premutation. Am J Med Genet 64 (2):323-328.

McConkie-Rosell A, Spiridigliozzi GA, Rounds K, Dawson DV, Sullivan JA, Burgess D, Lachiewicz AM. (1999). Parental attitudes regarding carrier testing in children at risk for fragile $\mathrm{X}$ syndrome. Am J Med Genet 82 (3):206-211.

McGue M, Bouchard TJ, Jr. (1998). Genetic and environmental influences on human behavioral differences. Annu Rev Neurosci 21:1-24.

Merenstein SA, Shyu V, Sobesky WE, Staley L, Berry-Kravis E, Nelson DL, Lugenbeel KA, Taylor AK, Pennington BF, Hagerman RJ. (1994). Fragile X syndrome in a normal IQ male with learning and emotional problems. J Am Acad Child Adolesc Psychiatry 33 (9):1316-1321.

Miezejeski CM, Jenkins EC, Hill AL, Wisniewski K, French JH, Brown WT. (1986). A profile of cognitive deficit in females from fragile X families. Neuropsychologia 24 (3):405-409.

Miller LJ, McIntosh DN, McGrath J, Shyu V, Lampe M, Taylor AK, Tassone F, Neitzel K, Stackhouse T, Hagerman RJ. (1999). Electrodermal responses to sensory stimuli in individuals with fragile X syndrome: a preliminary report. Am J Med Genet 83 (4):268-279.

Moutou C, Vincent MC, Biancalana V, Mandel JL. (1997). Transition from premutation to full mutation in fragile X syndrome is likely to be prezygotic. Hum Mol Genet 6 (7):971-979.

Moxley RT, 3rd, Ricker K. (1995). Proximal myotonic myopathy. Muscle Nerve 18 (5):557-558.

Murphy DG, Mentis MJ, Pietrini P, Grady CL, Moore CJ, Horwitz B, Hinton V, Dobkin CS, Schapiro 
MB, Rapoport SI. (1999). Premutation female carriers of fragile X syndrome: a pilot study on brain anatomy and metabolism [see comments]. J Am Acad Child Adolesc Psychiatry 38 (10): 1294-1301.

Murphy M. Bolton PF, Pickles A. Fombonne E, Piven J, Rutter M. (2000). Personality traits of the relatives of autistic probands. Psychol Med 30 (6):1411-1424.

Murray A, Webb J, Grimley S, Conway G, Jacobs P. (1998). Studies of FRAXA and FRAXE in women with premature ovarian failure. J Med Genet 35 (8):637-640.

Murray A, Ennis S. MacSwiney F, Webb J, Morton NE. (2000). Reproductive and menstrual history of females with fragile X expansions. Eur J Hum Genet 8 (4):247-252.

Musumeci SA, Ferri R, Elia M, Colognola RM, Bergonzi P, Tassinari CA. (1991). Epilepsy and fragile X syndrome: a follow-up study. Am J Med Genet 38 (2-3):511-513.

Myers GF, Mazzocco MM, Maddalena A, Reiss AL. (2001). No widespread psychological effect of the fragile $\mathrm{X}$ premutation in childhood: evidence from a preliminary controlled study. $J$ Dev Behav Pediatr 22 (6):353-359.

Naătănen R, Sokolov EN. (1992). Altention and brain function. Hillsdale (N.J.): Lawrence Erlbaum Associates.

Nielsen KB, Tommerup N, Poulsen H, Mikkelsen M. (1981). X-linked mental retardation with fragile $\mathrm{X}$. A pedigree showing transmission by apparently unaffected males and partial expression in female carriers. Hum Genet 59 (1):23-25.

Nolin SL, Glicksman A, Houck GE, Jr., Brown WT, Dobkin CS. (1994). Mosaicism in fragile X affected males. Am J Med Genet 51 (4):509-512.

Nolin SL, Houck GE, Jr., Gargano AD, Blumstein H, Dobkin CS, Brown WT. (1999). FMR1 CGGrepeat instability in single sperm and lymphocytes of fragile- $\mathrm{X}$ premutation males. Am J Hum Genet 65 (3):680-688.

Norremolle A, Sorensen SA, Fenger K, Hasholt L. (1995). Correlation between magnitude of CAG repeat length alterations and length of the paternal repeat in paternally inherited Huntington's disease. Clin Genet 47 (3):113-117.

Nurnberger JI, Jr., Goldin LR, Gershon ES: Genetics of psychiatric disorders. (1994). In: Winokur G, Clayton P, eds. The medical basis of psychiatry. Philadelphia: W.B. Saunders, 459-492.

Nyhan WL. (1972). Behavioral phenotypes in organic genetic disease. Presidential address to the Society for Pediatric Research, May 1, 1971. Pediatr Res 6 (1):1-9.

O'Brien G. (1992). Behavioural Phenotypy in Developmental Psychiatry. European Child and Adolescent Psychiatry Suppl. 1:1-61.

O'Brien G, Yule W. (1995). Behavioural Phenotypes. Cambridge: Cambridge University Press.

OMIM. (2002) Online Mendelian Inheritance in Man. Mc.Kusick-Nathans Institute for Genetic Medicine, Johns Hopkins University, Baltimore (MD), and National Center for Biotechnology Information, National Library of Medicine, Bethesda (MD). World Wide Web: http://www.ncbi.nlm.nih.gov/omim/.

Ono S, Kurisaki H, Sakuma A. Nagao K. (1995). Myotonic dystrophy with alveolar hypoventilation and hypersomnia: a clinicopathological study. J Neurol Sci 128 (2):225-231.

Ono S, Takahashi K, Jinnai K, Kanda F, Fukuoka Y, Kurisaki H, Mitake S, Inagaki T, Yamano T, Nagao K. (1998). Loss of serotonin-containing neurons in the raphe of patients with myotonic dystrophy: a quantitative immunohistochemical study and relation to hypersomnia. Neurology $50(2): 535-538$.

Opitz JM, Westphal JM, Daniel A. (1984). Discovery of a connective tissue dysplasia in the MartinBell syndrome. Am J Med Genet 17 (1):101-109.

Palmer BW, Boone KB, Chang L, Lee A, Black S. (1994). Cognitive deficits and personality patterns in maternally versus paternally inherited myotonic dystrophy. J Clin Exp Neuropsychol 16 (5):784-795.

Penrose LS. (1938). A clinical and genetic study of 1280 cases of mental defect. London

Perini GI, Colombo G, Armani M, Pellegrini A, Ermani M, Miotti M, Angelini C. (1989). Intellectual impairment and cognitive evoked potentials in myotonic dystrophy. $J$ Nerv Ment Dis 177 (12):750-754.

Perini GI, Menegazzo E, Ermani M, Zara M, Gemma A, Ferruzza E, Gennarelli M. Angelini C. (1999). Cognitive impairment and (CTG)n expansion in myotonic dystrophy patients. Biol 
Psychiatry 46 (3):425-431.

Petronis A, Kennedy JL. (1995). Unstable genes-unstable mind? Am J Psychiatry 152 (2):164-172.

Pflugfelder GO. (1998). Genetic lesions in Drosophila behavioural mutants. Behav Brain Res 95 (1):315

Philips AV. Timchenko LT. Cooper TA. (1998). Disruption of splicing regulated by a CUG-binding protein in myotonic dystrophy. Science 280 (5364):737-741.

Phillips MF, Steer HM, Soldan JR, Wiles CM, Harper PS. (1999). Daytime somnolence in myotonic dystrophy. J Neurol 246 (4):275-282.

Plomin R, Owen MJ, McGuffin P. (1994). The genetic basis of complex human behaviors. Science 264 (5166): 1733-1739.

Plomin R, DeFries JC, McClearn GE, Rutter M. (2000). Behavioral Genetics. New York: W.H. Freeman and Company.

Portwood MM. Wicks JJ, Lieberman JS, Fowler WM, Jr. (1984). Psychometric evaluation in myotonic muscular dystrophy. Arch Phys Med Rehabil 65 (9):533-536.

Portwood MM, Wicks JJ, Lieberman JS, Duveneck MJ. (1986). Intellectual and cognitive function in adults with myotonic muscular dystrophy. Arch Phys Med Rehabil 67 (5):299-303.

Pronk JC, Leschot NJ, Bijlsma EK, Beemer FA, Geraedts JP, Liebaers I. (1999): Leerboek medische genetica. Maarssen: Elsevier/Bunge.

Ragazzoni A. Pinto F. Taiuti R, Silveri MC. (1991). Myotonic dystrophy: an electrophysiological study of cognitive deficits. Can J Neurol Sci 18 (3):300-306.

Ragazzoni A, Amantini A, Lombardi M, Macucci M, Mascalchi M. Pinto F. (1993). Electric and CO2 laser SEPs in a patient with asymptomatic syringomyelia. Electroencephalogr Clin Neurophysiol 88 (4):335-338

Raven JC, Court JH. Raven J. (1984): Manual for Raven's Progressive Matrices and Vocabulary Scales. London: Lewis.

Refsum S, Lonnum A, Sjaastad O, Engeset A. (1967). Dystrophia myotonica. Repeated pneumoencephalographic studies in ten patients. Neurology 17 (4):345-348.

Reich W, Herjanic B, Welner Z, Gandhy PR. (1982). Development of a structured psychiatric interview for children: agreement on diagnosis comparing child and parent interview. $J$ Abn Child Psychol 10:325-336.

Reich W, Shayka J, Taibleson C. (1991). Diagnostic Interview for Children and Adolescents: Revised Parent Form (DICA-R-P). St.Louis: Washington University.

Reiss AL, Aylward E, Freund LS, Joshi PK, Bryan RN. (1991a). Neuroanatomy of fragile X syndrome: the posterior fossa. Ann Neurol 29 (1):26-32.

Reiss AL, Freund L, Tseng JE, Joshi PK. (1991b). Neuroanatomy in fragile X females: the posterior fossa. Am J Hum Genet 49 (2):279-288.

Reiss AL, Freund L. (1992). Behavioral phenotype of fragile X syndrome: DSM-III-R autistic behavior in male children. Am J Med Genel 43 (1-2):35-46.

Reiss AL, Freund L, Abrams MT, Boehm C, Kazazian H. (1993). Neurobehavioral effects of the fragile X premutation in adult women: a controlled study. Am J Hum Genet 52 (5):884-894.

Reiss AL, Kazazian HH, Jr., Krebs CM, McAughan A, Boehm CD, Abrams MT, Nelson DL. (1994a). Frequency and stability of the fragile X premutation. Hum Mol Genet 3 (3):393-398.

Reiss AL, Lee J, Freund L. (1994b). Neuroanatomy of fragile X syndrome: the temporal lobe. Neurology 44 (7): 1317-1324.

Reiss AL, Abrams MT, Greenlaw R, Freund L, Denckla MB. (1995). Neurodevelopmental effects of the FMR-1 full mutation in humans. Nat Med 1 (2):159-167.

Reyniers E, Martin JJ, Cras P, Van Marck E, Handig I, Jorens HZ, Oostra BA, Kooy RF, Willems PJ. (1999). Postmortem examination of two fragile X brothers with an FMR 1 full mutation. Am J Med Genel 84 (3):245-249.

Richards BW, Sylvester PE, Brooker C. (1981). Fragile X-linked mental retardation: the Martin-Bell syndrome. J Ment Defic Res 25 Pt 4:253-256.

Richards RI. (2001). Dynamic mutations: a decade of unstable expanded repeats in human genetic disease. Hum Mol Genet 10 (20):2187-2194.

Richardson SA. (1989). Issues in the definition of mental retardation and the representativeness of studies. Res Dev Disabil 10 (3):285-294. 
Ricker K. (1999). Myotonic dystrophy and proximal myotonic myophathy. J Neurol 246 (5):334-338.

Riddle JE, Cheema A, Sobesky WE, Gardner SC, Taylor AK, Pennington BF, Hagerman RJ. (1998). Phenotypic involvement in females with the FMRI gene mutation. Am J Ment Retard 102 (6):590-601.

Risch N. (1990). Linkage strategies for genetically complex traits. I. Multilocus models. Am J Hum Genet 46:222-228.

Risch N, Spiker D, Lotspeich L, Nouri N, Hinds D, Hallmayer J, Kalaydjieva L, McCague P, Dimiceli S, Pitts T, Nguyen L, Yang J, Harper C, Thorpe D, Vermeer S, Young H, Hebert J, Lin A, Ferguson J, Chiotti C, Wiese-Slater S, Rogers T, Salmon B, Nicholas P, Myers RM, et al. (1999). A genomic screen of autism: evidence for a multilocus etiology. Am J Hum Genet 65 (2):493-507.

Roig M, Balliu PR, Navarro C, Brugera R, Losada M. (1994). Presentation, clinical course, and outcome of the congenital form of myotonic dystrophy. Pediatr Neurol 11 (3):208-213.

Rosman NP, Rebeiz JJ. (1967). The cerebral defect and myopathy in myotonic dystrophy. A comparative clinicopathological study. Neurology 17 (11):1106-1112.

Rousseau F, Heitz D, Tarleton J, MacPherson J, Malmgren H, Dahl N, Barnicoat A, Mathew C, Mornet E, Tejada I, Maddalena A, Spiegel R, Schinzel A, Marcos J, Schorderet DF, Schaap T, Maccioni L. Russo S, Jacobs P. Schwartz C, Mandel JL. (1994a). A multicenter study on genotype-phenotype correlations in the fragile $\mathrm{X}$ syndrome, using direct diagnosis with probe StB 12.3: the first 2,253 cases. Am J Hum Genet 55 (2):225-237.

Rousseau F, Robb LJ, Rouillard P, Der Kaloustian VM. (1994b). No mental retardation in a man with $40 \%$ abnormal methylation at the FMR- 1 locus and transmission of sperm cell mutations as premutations. Hum Mol Genet 3 (6):927-930.

Rousseau F, Rouillard P, Morel ML, Khandjian EW, Morgan K. (1995). Prevalence of carriers of premutation-size alleles of the FMRI gene-- and implications for the population genetics of the fragile X syndrome [see comments]. Am J Hum Genet 57 (5): 1006-1018.

Rubinszlein JS, Rubinsztein DC, McKenna PJ, Goodburn S, Holland AJ. (1997). Mild myotonic dystrophy is associated with memory impairment in the context of normal general intelligence. J Med Genet 34 (3):229-233.

Rubinsztein JS, Rubinsztein DC, Goodburn S, Holland AJ. (1998). Apathy and hypersomnia are common features of myotonic dystrophy. J Neurol Neurosurg Psychiatry 64 (4):510-515.

Rugtveit J. (1986). X-linked mental retardation and infantile spasms in two brothers. Dev Med Child Neurol 28 (4):544-546.

Rutter M, Macdonald H. Le Couteur A. Harrington R, Bolton P. Bailey A. (1990). Genetic factors in child psychiatric disorders--Il. Empirical findings. J Child Psychol Psychiatry 31 (1):39-83.

Rutter M. (1994). Psychiatric genetics: research challenges and pathways forward. Am J Med Genet 54 (3): 185-198.

Rutter M, Simonoff E, Plomin R. (1996). Genetic influences on mild mental retardation: concepts, findings and research implications. J Biosoc Sci 28 (4):509-526.

Rutter M, Silberg J, OConnor T, Simonoff E. (1999a). Genetics and child psychiatry: II Empirical research findings. J Child Psychol Psychiatry 40 (1):19-55.

Rutter M, Silberg J, OConnor T, Simonoff E. (1999b). Genetics and child psychiatry: I Advances in quantitative and molecular genetics. J Child Psychol Psychiatry 40 (1):3-18.

Rutter M. (2000). Genetic studies of autism: from the 1970s into the millennium. J Abnorm Child Psychol 28 (1):3-14.

Sabaratnam M, Vroegop PG, Gangadharan SK. (2001). Epilepsy and EEG findings in 18 males with fragile X syndrome. Seizure 10(1):60-63.

Sarkar PS, Appukuttan B, Han J, lto Y, Ai C, Tsai W, Chai Y, Stout JT, Reddy S. (2000). Heterozygous loss of Six 5 in mice is sufficient to cause ocular cataracts. Nat Genet 25 (1):110114.

Savkur RS, Philips AV, Cooper TA. (2001). Aberrant regulation of insulin receptor alternative splicing is associated with insulin resistance in myotonic dystrophy. Nat Genet 29 (1):40-47.

Schatz J, Hamdan-Allen G. (1995). Effects of age and IQ on adaptive behavior domains for children with autism. J Autism Dev Disord 25 (1):51-60.

Schrander-Stumpel C, Howeler C, Jones M, Sommer A, Stevens C, Tinschert S, Israel J, Fryns JP. 
(1995). Spectrum of X-linked hydrocephalus (HSAS), MASA syndrome, and complicated spastic paraplegia (SPG1): Clinical review with six additional families. Am J Med Genet 57 (1): 107-116.

Schwartz C, Stevenson R. (2002) XLMR Update - April 2002. Greenwood Genetic Center. World Wide Web: http://www gge.org/xlmr.html.

Sergeant J. (2000). The cognitive-energetic model: an empirical approach to attention- deficit hyperactivity disorder. Neurosei Biobehav Rev 24 (1):7-12.

Shaw DJ, McCurrach M, Rundle SA, Harley HG, Crow SR, Sohn R. Thirion JP, Hamshere MG, Buckler AJ. Harper PS, et al. (1993). Genomic organization and transcriptional units at the myotonic dystrophy locus. Genomics 18 (3):673-679.

Sherman SL, Jacobs PA, Morton NE, Froster-Iskenius U, Howard-Peebles PN, Nielsen KB, Partington MW, Sutherland GR. Turner G, Watson M. (1985). Further segregation analysis of the fragile $X$ syndrome with special reference to transmitting males. Hum Genet 69 (4):289-299.

Simon EW, Finucane BM. (1996). Facial emotion identification in males with fragile X syndrome. Am $J$ Med Genel 67 (1):77-80.

Sinforiani E, Sandrini G, Martelli A, Mauri M, Uggetti C, Bono G, Nappi G. (1991). Cognitive and neuroradiological findings in myotonic dystrophy. Funct Neurol 6 (4):377-384.

Smalley SL. (1998). Autism and tuberous sclerosis. J Autism Dev Disord 28 (5):407-414.

Smits A. Smeets D, Dreesen J. Hamel B, de Haan A, van Oost B. (1992). Parental origin of the fra(X) gene is a major determinant of the cytogenetic expression and the CGG repeat length in female carriers. Am J Med Genet 43 (1-2):261-267.

Sobesky WE, Hull CE, Hagerman RJ. (1994a). Symptoms of schizotypal personality disorder in fragile X women. J Am Acad Child Adolesc Psychiatry 33 (2):247-255.

Sobesky WE, Pennington BF. Porter D, Hull CE, Hagerman RJ. (1994b). Emotional and neurocognitive deficits in fragile X. Am J Med Genet 51 (4):378-385.

Sobesky WE, Taylor AK, Pennington BF, Bennetto L, Porter D, Riddle J, Hagerman RJ. (1996). Molecular/clinical correlations in females with fragile X. Am J Med Genet 64 (2):340-345.

Sparrow SS, Balla DA, Cicchetti D. (1984). Vineland Adaptive Behavior Scales. Circle Pines, MN.: American Guidance Service.

Sreeram N, Wren C, Bhate M, Robertson P, Hunter S. (1989). Cardiac abnormalities in the fragile X syndrome. Br Heart J 61 (3):289-291.

SSBP. (2002) Society for the Study of Behavioural Phenotypes. World Wide Web: http://www psychiatry.cam ac.uk/SSBP/.

Steyaert J, Borghgraef M, Gaulthier C, Fryns JP, Van den Berghe H. (1992). Cognitive profile in adult, normal intelligent female fragile X carriers. Am J Med Genet 43 (1-2):116-119.

Steyaert J, Decruyenaere M, Borghgraef M, Fryns JP. (1994). Personality profile in adult female fragile X carriers: assessed with the Minnesota Multiphasic Personality Profile (MMPI). Am J Med Genet 51 (4):370-373.

Steyaert J, Borghgraef M, Legius E, Fryns JP. (1996). Molecular-intelligence correlations in young fragile X males with a mild CGG repeat expansion in the FMR1 gene. Am J Med Genet 64 (2):274-277.

Steyaert J, Umans S, Willekens D, Legius E, Pijkels E, de Die-Smulders C, Van den Berghe H, Fryns JP. (1997). A study of the cognitive and psychological profile in 16 children with congenital or juvenile myotonic dystrophy. Clin Genet 52 (3):135-141.

Steyaert J, de Die-Smulders C, Fryns JP, Goossens E, Willekens D. (2000). Behavioral phenotype in childhood type of dystrophia myotonica. Am J Med Genet 96 (6):888-889.

Steyaert J, Legius E, Borghgraef M, Fryns J-P. (2002). A distinct neurocognitive phenotype in female fragile X premutation carriers asssessed with visual attention tasks. Am J Med Genet In press.

Stinissen J, Willem PJ, Coetsier P, Hulsman WL. (1970). Handleiding bij de Nederlandse Bewerking van de Wechsler Adult Intelligence Scale (WAIS). Lisse: Swets \& Zeitlinger BV.

Stromme P, Valvatne K. (1998). Mental retardation in Norway: prevalence and sub-classification in a cohort of 30037 children born between 1980 and 1985. Acta Paediatr 87 (3):291-296.

Sutcliffe JS, Nelson DL, Zhang F, Pieretti M, Caskey CT, Saxe D, Warren ST. (1992). DNA methylation represses FMR-1 transcription in fragile X syndrome. Hum Mol Genet 1 (6):397400 . 
Sutherland GR. (1977). Fragile sites on human chromosomes: demonstration of their dependence on the type of tissue culture medium. Science 197 (4300):265-266.

Sutherland GR. (1978). Carrier detection in X-linked mental retardation. Med J Aust 2 (14):624.

Sutherland GR, Gedeon A, Kornman L, Donnelly A, Byard RW, Mulley JC, Kremer E, Lynch M, Pritchard M, Yu S, et al. (1991). Prenatal diagnosis of fragile X syndrome by direct detection of the unstable DNA sequence [see comments]. N Engl J Med 325 (24):1720-1722.

Swillen A, Devriendt K, Legius E, Eyskens B, Dumoulin M, Gewillig M, Fryns JP. (1997). Intelligence and psychosocial adjustment in velocardiofacial syndrome: a study of 37 children and adolescents with VCFS. JMed Genet 34 (6):453-458.

Swillen A, Devriendt K, Legius E, Prinzie P, Vogels A, Ghesquiere P, Fryns JP. (1999). The behavioural phenotype in velo-cardio-facial syndrome (VCFS): from infancy to adolescence. Genet Couns 10 (1):79-88.

Swillen A, Vogels A, Devriendt K, Fryns JP. (2000). Chromosome 22q11 deletion syndrome: update and review of the clinical features, cognitive-behavioral spectrum, and psychiatric complications. Am JMed Genet 97 (2):128-135.

SYSTAT®. (1997): SYSTAT 7.0 for Windows.: SPSS Inc., Chicago.

Szymanski L, King BH. (1999). Practice parameters for the assessment and treatment of children, adolescents, and adults with mental retardation and comorbid mental disorders. American Academy of Child and Adolescent Psychiatry Working Group on Quality Issues. J Am Acad Child Adolesc Psychiatry 38 (12 Suppl):5S-31S.

Tapscott SJ, Thornton CA. (2001). Biomedicine. Reconstructing myotonic dystrophy. Science 293 (5531):816-817.

Tassone F, Hagerman RJ, Gane LW, Taylor AK. (1999a). Strong similarities of the FMR1 mutation in multiple tissues: postmortem studies of a male with a full mutation and a male carrier of a premutation. Am J Med Genet 84 (3):240-244.

Tassone F, Longshore J, Zunich J. Steinbach P, Salat U, Taylor AK. (1999b). Tissue-specific methylation differences in a fragile X premutation carrier. Clin Genet 55 (5):346-351.

Tassone F, Hagerman RJ, Loesch DZ, Lachiewicz A, Taylor AK, Hagerman PJ. (2000a). Fragile X males with unmethylated, full mutation trinucleotide repeat expansions have elevated levels of FMRI messenger RNA. Am J Med Genet 94 (3):232-236.

Tassone F, Hagerman RJ, Taylor AK, Gane LW, Godfrey TE, Hagerman PJ. (2000b). Elevated levels of FMRI mRNA in carrier males: a new mechanism of involvement in the fragile-X syndrome. Am J Hum Genet 66 (1):6-15.

Tassone F, Hagerman RJ, Taylor AK, Mills JB, Harris SW, Gane LW, Hagerman PJ. (2000c). Clinical involvement and protein expression in individuals with the FMR1 premutation. Am J Med Genet 91 (2):144-152.

Theuns J, Van Broeckhoven C. (2000). Transcriptional regulation of Alzheimer's disease genes: implications for susceptibility. Hum Mol Genet 9 (16):2383-2394.

Thompson NM, Gulley ML, Rogeness GA, Clayton RJ, Johnson C, Hazelton B, Cho CG, Zellmer VT. (1994). Neurobehavioral characteristics of CGG amplification status in fragile $\mathrm{X}$ females. Am JMed Genet 54 (4):378-383.

Thompson NM, Rogeness GA, McClure E, Clayton R, Johnson C. (1996). Influence of depression on cognitive functioning in Fragile X females. Psychiatry Res 64 (2):97-104.

Thornton C. (1999). The myotonic dystrophies. Semin Neurol 19 (1):25-33.

Tuikka RA, Laaksonen RK, Somer HV. (1993). Cognitive function in myotonic dystrophy: a followup study. Eur Neurol 33 (6):436-441.

Turner G, Webb T, Wake S, Robinson H. (1996). Prevalence of fragile X syndrome. Am J Med Genet 64 (1):196-197.

Turnpenny P. Clark C. Kelly K. (1994). Intelligence quotient profile in myotonic dystrophy, intergenerational deficit, and correlation with CTG amplification. J Med Genet 31 (4):300305.

Uchida IA, Joyce EM. (1982). Activity of the fragile X in heterozygous carriers. Am J Hum Genet 34 (2):286-293.

Uzielli ML, Guarducci S, Lapi E, Cecconi A, Ricci U, Ricotti G, Biondi C, Scarselli B, Vieri F, Scarnato P, Gori F, Sereni A. (1999). Premature ovarian failure (POF) and fragile X 
premutation females: from $\mathrm{POF}$ to to fragile $\mathrm{X}$ carrier identification, from fragile $\mathrm{X}$ carrier diagnosis to POF association data. Am J Med Genet 84 (3):300-303.

van der Meche FG, Bogaard JM, van der Sluys JC, Schimsheimer RJ, Ververs CC, Busch HF, (1994). Daytime sleep in myotonic dystrophy is not caused by sleep apnoea. $J$ Neurol Neurosurg Psychiatry 57 (5):626-628.

Van Haasen PP, De Bruyn EEJ, Pijl YJ, Poortinga YH, Spelberg HC, Vander Steene G, Coetsier P, Spoelders-Claes R. Stinissen J. (1974). Wechsler intelligence scale for children - Revised. Nederlandstalige uitgave. Lisse: Swets \& Zeitlinger.

Van Spaendonck KP. Ter Bruggen JP, Weyn Banningh EW, Maassen BA, Van de Biezenbos JB, Gabreels FJ. (1995). Cognitive function in early adult and adult onset myotonic dystrophy. Acta Neurol Scand 91 (6):456-461.

Vander Steene G, Van Haasen PP, De Bruyn EE, Coetsier P, Pijl YJ, Poortingha YH, Spelberg HC, Stinissen J. (1986). Wechsler Intelligence Scale for Children-Revised Nederlandstalige uitgave, Lisse: Swets \& Zeitlinger, BV.

Vanier TM. (1960). Dystrophia myotonica in childhood. Br Med J 2:1284-1288.

Verhoeven WM, Curfs LM, Tuinier S. (1998). Prader-Willi syndrome and cycloid psychoses. $J$ Intellect Disabil Res 42 (Pt 6):455-462.

Verhoeven WM. Tuinier S, Curfs LM. (2000). Prader-Willi psychiatric syndrome and Velo-CardioFacial psychiatric syndrome. Genet Couns 11 (3):205-213.

Verhoeven WM. Tuinier S. (2001). Two steps forward, one step back; paradigmatic changes in psychiatry. J Neural Transm 108 (6):617-627.

Verhoeven WM, Curfs LM. Tuinier S. (2002a). Prader-Willi syndrome and atypical psychoses. Eur Psychiatry 17 Suppl 1:171.

Verhoeven WM, Tuinier S, Vogels A, Swillen A, Curfs LM, Frijns JP. (2002b), The psychopathological phenotype of Velo-Cardio-Facial Syndrome. Eur Psychiatry 17 Suppl 1:154.

Verhulst FC, Koot JM, Akkerhuis GW, Veerman JW. (1990). Handboek voor de Child Behaviour Checklist. Assen: Van Gorcum.

Verhulst FC, Van der Ende J, Koot HM. (1996). Handleiding voor de CBCL/4-18. Rotterdam: Sophia Kinderziekenhuis, Erasmus Universiteit Rotterdam.

Verkerk AJ, Pieretti M, Sutcliffe JS, Fu YH, Kuhl DP, Pizzuti A, Reiner O, Richards S, Victoria MF, Zhang FP. (1991). Identification of a gene (FMR-1) containing a CGG repeat coincident with a breakpoint cluster region exhibiting length variation in fragile X syndrome. Cell 65 (5):905914.

Ververs CC, Van der Meche FG, Verbraak AF, van der Sluys HC, Bogaard JM. (1996). Breathing pattern awake and asleep in myotonic dystrophy. Respiration 63 (1):1-7.

Vianna-Morgante AM. (1999). Twinning and premature ovarian failure in premutation fragile X carriers [letter]. Am JMed Genet 83 (4):326.

Vincent JB, Yuan QP, Schalling M, Adolfsson R, Azevedo MH, Macedo A, Bauer A, DallaTorre C, Medeiros HM, Pato MT, Pato CN, Bowen T, Guy CA, Owen MJ, ODonovan MC, Paterson AD, Petronis A, Kennedy JL. (2000). Long repeat tracts at SCA8 in major psychosis. Am J Med Genet 96 (6):873-876.

Vincent MC, Heitz F, Tricoire J, Bourrouillou G, Kuhlein E, Rolland M, Calvas P. (1999). 22q11 deletion in DGS/VCFS monozygotic twins with discordant phenotypes. Genet Couns 10 (1):43-49.

Vogels A, Verhoeven WM, Tuinier S, DeVriendt K, Swillen A, Curfs LM, Frijns JP. (2002). The psychopathological phenotype of velo-cardio-facial syndrome. Ann Genet 45 (2):89-95.

Wang PP, Woodin MF, Kreps-Falk R, Moss EM. (2000). Research on behavioral phenotypes: velocardiofacial syndrome (deletion 22q11.2). Dev Med Child Neurol 42 (6):422-427.

Warren ST. (1997). Trinucleotide Repetition and fragile X syndrome. Hosp Pract 32 (4): 73.

Weller S, Gartner J. (2001). Genetic and clinical aspects of X-linked hydrocephalus (L1 disease): Mutations in the LICAM gene. Hum Mutat 18 (1):1-12.

Welner Z, Reich W, Herjanic B, Jung KG, Amado H. (1987). Reliability, validity, and parent-child agreement studies of the Diagnostic Interview for Children and Adolescents (DICA). J Am Acad Child Adolesc Psychiatry 26:649-653. 
Wieringa B. (1994). Myotonic dystrophy reviewed: back to the future? Hum Mol Genet 3 (1):1-7.

Willemsen R, Olmer R, De Diego Otero Y, Oostra BA. (2000). Twin sisters, monozygotic with the fragile X mutation, but with a different phenotype. J Med Genet 37 (8):603-604.

Winchester CL, Ferrier RK, Sermoni A, Clark BJ, Johnson KJ. (1999). Characterization of the expression of DMPK and SIX5 in the human eye and implications for pathogenesis in myotonic dystrophy. Hum Mol Genet 8 (3):481-492.

Winsberg BG, Comings DE. (1999). Association of the dopamine transporter gene (DAT1) with poor methylphenidate response. J Am Acad Child Adolesc Psychiatry 38 (12):1474-1477.

Wohrle D, Hirst MC, Kennerknecht I, Davies KE, Steinbach P. (1992). Genotype mosaicism in fragile $X$ fetal tissues. Hum Genet 89 (1):114-116.

Wohrle D, Salat U, Hameister H. Vogel W, Steinbach P. (2001). Demethylation, reactivation, and destabilization of human fragile $x$ full-mutation alleles in mouse embryocarcinoma cells. Am J Hum Genet 69 (3):504-515.

Wolff PH, Gardner J, Lappen J, Paccia J, Meryash D. (1988). Variable expression of the fragile X syndrome in heterozygous females of normal intelligence. Am J Med Genet 30 (1-2):213-225.

Woodward JB, 3rd, Heaton RK, Simon DB, Ringel SP. (1982). Neuropsychological findings in myotonic dystrophy. J Clin Neuropsychol 4 (4):335-342.

Woolf CM. (1995). Influence of stochastic events on the phenotypic variation of common white leg markings in the Arabian horse: implications for various genetic disorders in humans. $J$ Hered $86(2): 129-135$.

Yu S, Pritchard M, Kremer E, Lynch M, Nancarrow J, Baker E, Holman K, Mulley JC, Warren ST, Schlessinger D, et al. (1991). Fragile X genotype characterized by an unstable region of DNA. Science 252 (5010):1179-1181. 


\section{Summary}

\section{a) Part I: introduction and literature}

Behavioural genetics developed as a new science in the $20^{\text {th }}$ century. It links quantitative, molecular and clinical genetics with behavioural sciences. One aim of behavioural genetics is to unravel the genetic mechanisms that underlie neuropsychiatric disorders, such as autism, mental retardation and others. Only a few pathofysiological pathways between gene and behaviour have been demonstrated until now. Quantitative genetics point at the important role of polygenic heredity in combination with environmental factors in the development of most behaviour characteristics and neuropsychiatric disorders. According to this model, an array of genes (Quantitative Trait Loci, QTL) each partly contribute to the development of a trait or disorder. Molecular genetic research based on QTL models has not yet been able to expose much of the molecular mechanisms underlying neuropsychiatric disorders and the phenotypical variance observed in these disorders. De expression of a single gene is very complex en can in some genes vary widely. In models involving a large number of genes, the complexity and variance will increase in an exponential way. This problem will only increase if most of the involved genes are still unknown. In chapter 3 of this thesis, we advocate that research on the effect of single genes on pathology of behaviour and the variance herein, is fundamental for research with polygenic models in combination with environmental factors. The impact of one gene (defect) on neuropsychiatric disorders is almost never a none-or-all phenomenon. Molecular mechanisms and stochastic effects in the development of the brain may result in a wide variance in the impact of a single gene (defect) on the phenotype. Dynamic mutations are one of these molecular mechanisms. These consist of an abnormal expansion of a poly-, mostly tri- nucleotide repeat. Such trinucleotide repeats are present at several sites in the genome of normal individuals. Different abnormal repeat lengths can be associated with differences in the severity of the disorder, a different age at onset of the disorder, or even to different disorders. Consequently, in dynamic mutation disorders, the same type of mutation in the same gene may be associated with important differences in the phenotype.

We studied the effect of dynamic mutations on the behavioural phenotype in two genetic conditions: Fragile X Syndrome (FXS) and Myotonic Dystrophy (Dystrophia myotonica, DM). In FXS (chapter 4), there are two different forms of the dynamic mutation in the FMRI 
gene on $\mathrm{Xq} 27.3$ : the premutation and the full mutation. The premutation consists of an abnormal expansion of a trinucleotide repeat (55-200 CGG repeats), which does not stop transcription and translation of the gene. Until recently, it was supposed that the premutation did not affect the phenotype. In the full mutation (> 200 CGG repeats), hypermethylation occurs in the promoter region of the gene, and transcription stops. The FMR1 protein cannot be produced anymore. This protein has an important role in growth and differentiation of neurones and dendrites. Male carriers of the full mutation will usually have the classical fragile X phenotype: moderate mental retardation, craniofacial features, and macro-orchidism. More than $50 \%$ of the female full mutation carriers have a milder expression of the same phenotype.

DM (chapter 5) is a progressive muscle disorder with multisystemic involvement. It has an autosomal dominant inheritance. Progressive muscle wasting and myotonia are associated with symptoms in the eye, the heart, the gastro-intestinal tract and other systems. The dynamic mutation in an untranslated region of the $D M P K$ gene on chromosome $19 \mathrm{q} 13$ consists of a CTG trinucleotide repeat of variable length. Four subtypes or phenotypes are differentiated, based on age-at-onset and severity. To some extent, these phenotypes correlate with different CTG repeat lengths. The four phenotypes are: de congenital form of DM, the juvenile or childhood form, the adult or classical form, and the late onset or mild form. The congenital form is systematically associated with mental retardation, and the adult form with increasing apathy and somnolence. These findings suggested that in DM the central nervous system is probably affected.

\section{b) Part II: research in Fragile X Syndrome}

In chapter 6 to 8 , we report research findings that have led to new insights in the genebehaviour relation in FXS. (1) In a group of 11 obligate carriers of the fragile X gene (chapter 6), we found a discrepancy between measured intelligence and level of education and job, as well as a number of mild cognitive deficits. This suggests that even in clinically non-affected female carriers, the gene defect may influence the central nervous system. (2) In chapter 7, we describe seven boys with the full mutation, but with a milder cognitive impairment than what is usually observed. Hypermethylation of the promoter region of the FMRI gene proved to be incomplete in these boys, and the degree of hypermethylation correlated negatively with full scale IQ. This finding demonstrated indirectly that there is a relation between degree of transcription of the FMRI gene into messenger RNA and IQ in males with FXS. Unfortunately, we did not have the opportunity to measure FMR1 protein production in these 
subjects. (3) In chapter 8, we describe a group of female carriers of the fragile $\mathrm{X}$ premutation who display neurocognitive characteristics that are both different from what is observed in normal female controls and in full mutation carriers. We observed differences in controlled information processing requiring focused attention in approximately $18 \%$ of these premutation carriers. Although these characteristics do not seem to have clinical implications. they suggest that in a substantial part of the female carriers the premutation has an effect on the development of the central nervous system. These findings are in line with recent gynaecological findings, which show that in approximately $20 \%$ of the female carriers the premutation leads to premature ovarian failure. This suggests that expansions of the CGG repeat in the FMRI gene may result in two different phenotypes: one associated with the premutation, and one with the full mutation.

\section{c) Part II: research in Myotonic Dystrophy}

In chapter 9 we report the results of standardized cognitive and behaviour testing in a referred group of 14 youngsters with juvenile DM and two youngsters with congenital DM. All subjects had an IQ below average. A significant number of them are socially withdrawn and/or have attention problems. We observed a mild decline of intelligence in the two subjects with congenital DM. In chapter 10 we report a replication and refinement of the previous study in 24 not clinically referred subjects with juvenile DM. A significant number of these subjects have behaviour problems in the child psychiatric range, principally ADHD and anxiety disorders. In this group too, average intelligence was significantly below the population mean, and 17 of the 21 youngsters who were still at school, had been placed in special education programs because of academic problems. In almost all these youngsters, academic problems had preceded neuromuscular symptoms. Unlike the predominantly maternal inheritance of the mutation found in congenital DM, in these subjects with juvenile DM paternal inheritance was as frequent as maternal inheritance. Remarkably, a number of subjects had inherited the mutation from an unaffected parent with a relatively small mutation. In some of these cases, DM had not been diagnosed in the core family yet, but only in more distant relatives. All these findings are quite different from the clinical picture in classical type DM. Referral bias is unlikely the cause of this as the group consisted of nonclinical subjects. The findings demonstrate the wide variance in the DM phenotype. It draws attention to the fact that one should always think of the possibility of juvenile DM when there is a family history of the condition, even if the parents are unaffected. On the other hand, learning and behaviour difficulties should receive the necessary attention in youngsters in 
whom juvenile DM has been diagnosed, even if their neuromuscular symptoms are still minimal. The finding that behaviour and cognitive problems often come before neuromuscular complaints is a strong argument that suggest that DM affects the brain directly. Not much is known about the pathofysiology of brain involvement in DM, en to clarify this further, molecular research, brain imaging and longitudinal studies in DM are necessary.

\section{d) Part III: Conclusions}

The research presented in this thesis illustrates the phenotypical variation that may exist in the expression of a mutation in a single gene. In FXS, molecular differences in the mutation have important effects on the phenotype: female carriers have two different phenotypes depending on the length of the mutation - premutation or full mutation-, and each of these phenotypes is different from what is found in the normal population. In male FXS full mutation carriers, cognitive impairment is strongly determined by another molecular variable: the degree of methylation in the promoter region of the gene. In DM, we saw that juvenile DM has a different presentation than the classical form of DM. The effect of the mutation length on the occurrence of two different phenotypes is not so distinct as in FXS. It is likely that other molecular, stochastic, or perhaps environmental factors play a role in the development towards the one or the other phenotype. 


\section{Samenvatting}

\section{e) Deel 1 : inleiding en literatuar}

In de twintigste eeuw ontstond de gedragsgenetica als nieuwe wetenschap. $\mathrm{Zij}$ vormt een brug tussen de kwantitatieve, de moleculaire en de klinische genetica enerzijds, en de gedragswetenschappen anderzijds. Een belangrijk doel van de gedragsgenetica is het ontrafelen van genetische mechanismen die een rol spelen in het ontstaan van neuropsychiatrische aandoeningen, o.m. psychiatrische ontwikkelingsstoornissen bij kinderen. Tot op heden werden slechts een beperkt aantal pathofysiologische mechanismen tussen genen en gedrag aangetoond. De bevindingen van de kwantitatieve genetica wijzen in de richting van een hoofdzakelijk polygene overerving van de meeste gedragskenmerken en stoornissen, in combinatie met milieu-invloeden. Verschillende genen (Quantitative Trait Loci, QTL) zouden een deel van het ontstaan van een kenmerk of stoornis verklaren. Moleculair genetisch onderzoek dat op dergelijke modellen gebaseerd is heeft tot nu echter weinig kunnen verklaren van het ontstaan van en de variantie binnen neuropsychiatrische ziektebeelden. De expressie van één enkel gen is al zeer complex en kan bij een aantal genen erg wisselend zijn. In een model met een groot aantal genen zullen complexiteit en variantie exponentieel toenemen met het aantal genen. Dit probleem wordt nog groter wanneer de meerderheid van de genen in een dergelijk model nog onbekend zijn. Wij betogen dan ook (hoofdstuk 3) dat onderzoek naar de invloeden van één enkel gen op het gedrag en de ontwikkeling, en naar de oorzaken van de variantie hierin, een onmisbaar fundament is voor het onderzoek van complexe modellen met meerdere genen in combinatie met milieufactoren. De invloed van één gen op het ontstaan van neuropsychiatrische stoornissen, is meestal geen alles-of-niets fenomeen. Diverse moleculaire mechanismen en stochastische effecten in de ontwikkeling van de hersenen leiden tot grote verschillen in de impact van een genafwijking op het fenotype. Eén van deze mechanismen vormen de zogenaamde dynamische mutaties: pathologische mutaties in een gen die bestaan uit een abnormale expansie van een anderzijds normale poly- en meestal tri-nucleotide repeat. Verschillende expansielengtes kunnen leiden tot verschillen in de ernst van het fenotype, verschillen in de beginleeftijd waarop de stoornis zich manifesteert, of soms tot totaal verschillende pathologische fenotypes. Eén type mutatie in hetzelfde gen is hier dus geassocieerd met belangrijke verschillen in het fenotype. 
Wij onderzochten het effect van dynamische mutaties in twee genetische aandoeningen: het Fragiel-X Syndroom (FXS) en Myotone Dystrofie (Dystrophia Myotonica, DM). Bij FXS (hoofdstuk 4) worden twee vormen van de fragiel-X mutatie in het FMRI-gen op chromosoom Xq27.3 onderscheiden: de premutatie en de volledige mutatie. Bij de premutatie is er sprake van een abnormale expansie van de trinucleotide sequentie (55-200 CGG repeats), waarbij transcriptie en translatie van het gen nog mogelijk zijn. Tot dusver werd aangenomen dat dit geen effect had op het fenotype. Bij de volledige mutatie (>200 CGG repeats) treedt een hypermethylatie op in de promotorregio van het gen, waardoor transcriptie niet meer mogelijk is. Het FMRI-eiwit wordt niet meer aangemaakt. Dit eiwit speelt een rol in de groei en differentiatie van neuronen en hun dendrieten. Nagenoeg alle mannelijke dragers van de volledige mutatie vertonen het klassieke FXS fenotype -matige mentale retardatie, macroörchidie, craniofaciale kenmerken- en ruim de helft van de vrouwelijke draagsters hebben een mildere expressie van dit fenotype.

DM (hoofdstuk 5) is een autosomaal dominante aandoening, die gekenmerkt wordt door een progressieve spierdystrofie gepaard met myotonie, en aantasting van verschillende systemen: oog, maag-darm, hart en andere. De dynamische mutatie in het DMPK-gen op chromosoom $19 q 13$ bestaat uit een CTG-trinucleotide repeat van variabele lengte. Op basis van ernst en aanvangsleeftijd van de aandoening worden vier verschillende fenotypes beschreven, die in beperkte mate correleren met de expansielengte van de CTG-repeat. Deze fenotypes zijn: de congenitale vorm, de juveniele vorm, de adulte vorm (of klassiek fenotype), en de laattijdige of milde vorm. Bekend was dat de congenitale vorm gepaard gaat met mentale retardatie, en dat bij de volwassen vorm meestal toenemende apathie en somnolentie optreden. Deze bevindingen wijzen erop dat er bij DM ook sprake kan zijn van een aantasting van het centraal zenuwstelsel.

\section{f) Deel II: onderzoeksbevindingen bij Fragiel Xsyndroom}

In hoofdstukken 6 t.e.m. 8 worden een aantal onderzoeksbevindingen gerapporteerd die tot nieuwe inzichten hebben geleid op het vlak van gen-gedragsrelaties in FXS. (1) Bij een groep van 11 obligate draagsters van het fragiel-X gen vonden wij een discrepantie tussen gemeten intelligentie en niveau van scholing en functioneren, evenals een aantal milde cognitieve afwijkingen. Dit suggereerde dat ook bij klinisch niet-aangetaste draagsters het gen toch een invloed kan hebben op het centraal zenuwstelsel. (2) In hoofdstuk 7 beschreven wij 7 jongens met de volledige fragiel-X mutatie, maar met mildere cognitieve aantasting dan wat doorgaans vastgesteld wordt. Bij hen bleek dat er geen of slechts gedeeltelijke 
hypermethylatie in de promotorregio van $F M R I$-gen was, en dat de gemeten intelligentie negatief correleerde met de graad van hypermethylatie. Deze bevinding pleitte ervoor dat een verband bestaat tussen de transcriptie van het FMRI-gen naar messenger RNA, en de intelligentie van personen met FXS. Wij waren helaas niet in de gelegenheid om het FMRIeiwit bij deze jongens te bepalen. (3) In hoofdstuk 8 beschreven wij een groep vrouwelijke draagsters van de fragiel-X premutatie, die neurocognitieve kenmerken hebben die zowel verschillen van wat vastgesteld wordt bij de normale vrouwelijke populatie, als van wat vastgesteld wordt bij draagsters van de volledige mutatie. Deze afwijkingen waren op het vlak van gecontroleerde informatieverwerking met gefocuste aandacht, en we troffen ze aan bij circa $18 \%$ van de draagsters van de premutatie. Alhoewel het hier ging om een afwijking die niet onmiddellijk klinische implicaties lijkt te hebben, suggereerden deze bevindingen dat bij een substantieel deel van de draagsters, de premutatie een invloed heeft op de ontwikkeling van het centraal zenuwstelsel. Deze bevinding ligt in de lijn van eerdere gynecologische bevindingen, die aantonen dat bij circa $20 \%$ van de draagsters de premutatie leidt tot vroegtijdige menopauze. Dit suggereert dat expansies van de CGG-repeat in het FMRI-gen kunnen leiden tot twee verschillende fenotypes: één geassocieerd aan de premutatie, en één geassocieerd aan de volledige mutatie.

\section{g) Deel II: onderzoeksbevindingen bij Myotone Dystrofie}

In hoofdstuk 9 rapporteerden we het gestandaardiseerd cognitief en gedragsonderzoek bij een klinische groep van 14 jongeren met juveniele DM en twee jongeren met congenitale DM. De gemeten intelligentie was bij alle subjecten benedengemiddeld. Een significant aantal subjecten was sociaal teruggetrokken en/of heeft aandachtsstoornissen. Bij de twee subjecten met congenitale DM werd longitudinaal een lichte daling van de gemeten intelligentie vastgesteld. In hoofdstuk 10 beschreven we een verfijning van dit onderzoek, waarbij het gedrag bij een niet-klinische groep van 24 subjecten met juveniele DM onderzocht werd. Hierbij bleek dat een belangrijk aantal subjecten gedragsproblemen had in de kinderpsychiatrische range, voornamelijk ADHD of angststoornissen. Ook bij deze groep was de gemiddelde intelligentie significant beneden het populatiegemiddelde, en 17 van de 21 jongeren die nog onderwijs volgden, waren wegens leermoeilijkheden naar speciaal onderwijs verwezen. Bij nagenoeg al deze jongeren gingen leerproblemen vooraf aan enige neuromusculaire klachten. In tegenstelling tot wat doorgaans wordt vastgesteld bij congenitale DM, was er bij deze groep geen overwegend maternele overerving van de mutatie, maar was deze even frequent van paternele oorsprong. Opvallend was dat in deze groep een aantal 
jongeren de mutatie overerfden van een niet-aangetaste ouder met een relatief kleine mutatie. De aandoening was dan niet eerder bekend in het gezin, maar alleen in de verdere familie.

De combinatie van deze bevindingen verschilt erg van de klassieke presentatie van DM. Het is onwaarschijnlijk dat dit aan referral bias kan worden toegeschreven, aangezien de groep niet-klinisch was gerekruteerd. Eén en ander toont de grote variatie in het DM-fenotype aan, en onderstreept dat aan de mogelijkheid van juveniele DM gedacht moet worden in risicofamilies. Bij jongeren met juveniele DM dient aandacht te worden besteed aan psychologische, psychiatrische en leerproblemen, ook als zij nog maar minimale neuromusculaire symptomen hebben. De bevindingen bij juveniele DM, in het bijzonder de bevinding dat leerproblemen de spierklachten vaak voorafgaan, tonen aan dat DM het centraal zenuwstelsel aantast onafhankelijk van de spiersymptomen. Er is nog maar weinig bekend over het pathofysiologisch mechanisme van deze aantasting, en om dit verder te onderzoeken zijn moleculair onderzoek, brain imaging en longitudinaal onderzoek bij DM nodig.

\section{h) Deel III: Conclusies}

Het onderzoek beschreven in dit proefschrift onderstreept de fenotypische variatie die bestaat in de expressie van mutaties in één enkel gen. Bij FXS zien we dat moleculaire verschillen in een genafwijking belangrijke gevolgen kunnen hebben op het fenotype: bij vrouwelijke draagsters van de fragiel-X mutatie zijn er twee kwalitatief verschillende fenotypes in functie van de lengte van de mutatie (premuatie versus volledige mutatie), die elk verschillen van het fenotype bij de normale populatie. Bij mannelijke dragers van de volledige mutatie zijn de cognitieve beperkingen sterk afhankelijk van een andere moleculaire variabele: de graad van hypermethylatie in de promotor van het gen. Bij DM zien we dat een bepaalde fenotypische vorm van de aandoening, met name de juveniele vorm, een beduidend verschillende klinische presentatie heeft vergeleken met de klassieke vorm van DM. Andere moleculaire, stochastische, en eventueel omgevingsfactoren spelen hier wellicht een rol in het ontstaan van het één of het andere fenotype. 


\section{Dankwoord}

Het uitspreken van een dankwoord lijkt in het begin van de weg naar een proefschrift misschien een formaliteit. Bij het beèindigen van het proefschrift heb je er sterk behoefte aan. Vooreerst wil ik mijn promotoren danken. Beste Jean-Pierre, prof. dr. J.-P. Fryns, je vond het steeds vanzelfsprekend dat in de klinische genetica ook naar psychologische en gedragsaspecten gekeken moet worden, en dat dit een volwaardig onderzoeksaspect is. Ook een psychiater bij wetenschappelijk onderzoek begeleiden vond je heel gewoon. Wat dit proefschrift betreft, wist je echter dat van een lange wandeling niet alleen de eerste, maar elke stap gezet dient te worden. En je liet dan ook nooit na aanmoedigingen te geven, en in mindere tijden het tempo te doseren. Steeds had je vertrouwen, en geduld.

Voor dit geduld en vertrouwen ben ik ook jou Joep, prof.dr. J.P.M.Geraedts, bijzonder dankbaar. Het heeft mij toegelaten de weg op mijn manier te gaan en hierdoor tot inzichten te komen die ik niet had gehad als ik de kortste weg had gevolgd. Joep, jij zorgde als vanzelfsprekend voor de nodige aanstellingen en financièn, zodat ik zonder zorgen deze weg kon gaan.

Willem, prof. dr. W.M.A Verhoeven, je kwam er later bij. Maar meteen sprong je in actie en ik dank je voor de opmerkingen en andere klemtonen die je als collega psychiater legde toen het proefschrift gestalte begon te krijgen.

Connie, prof. dr. C.T.R.M. Schrander-Stumpel, en Christine, dr. C.E.M. de Die-Smulders, jullie waren een soort zusters of gidsen, die feilloos uittekenden welke hindernissen je ontmoet bij het schrijven van een proefschrift, en mij steeds deden vertrouwen in de goede afloop. Ik ben jullie hiervoor heel dankbaar.

Collega's in Leuven en Maastricht, in het bijzonder Martine Borghgraef, Diane Willekens, en Eric Legius, mijn dank voor jullie inhoudelijke bijdragen aan het onderzoek. Jullie zorgden met zijn allen echter ook voor een goed sfeer, waardoor de dagen op genetica een soort wekelijks uitstapje zijn, hoe hard er ook gewerkt wordt.

Mijn dank aan Elly Pijkels, Maureen Holvoet, en Vera Hovers, die de families contacteerden die aan het onderzoek deelnamen.

Bijzondere dank aan de families met fragiel $\mathrm{X}$ syndroom of myotone dystrofie, die steeds met belangstelling deelnamen aan onderzoek.

Thomy de Ravel, je las dit proefschrift door en voorzag het waar nodig van taalkundige en andere commentaar. Rita Logist, dank voor je goede zorgen voor de manuscripten en alle administratie die erbij hoort.

Mijn vader en moeder, vaak heb ik aan jullie gedacht. Jullie geloof heeft mij hier gebracht.

Marie, vaak was ik niet helemaal bij als je mij nodig had, en je aanvaardde het.

Charlotte, ....jij was er de laatste jaren steeds. 


\section{Curriculum Vitae}

Jean Steyaert werd geboren op 15 april 1959 in Gent (Belgiē). Hij behaalde het humanioradiploma in 1977 aan het Sint-Jozefscollege in Hasselt, en studeerde vervolgens geneeskunde aan de Rijksuniversiteit Gent en de Katholieke Universiteit Leuven. In 1984 behaalde hij aan deze laatste universiteit de titel van Doctor in de Genees-, Heel-, en Verloskunde. Onder leiding van prof.dr. K. Pyck specialiseerde hij in de kinder- en jeugdpsychiatrie. Hij werd erkend als geneesheer-specialist in de neuropsychiatrie in 1989, en als revalidatiearts in 1992. Hij is was staflid in de afdeling kinder- en jeugdpsychiatrie van de Universitaire Ziekenhuizen Leuven van 1989 tot 1992. Van 1992 tot 2001 was hij kinderpsychiater bij het Kinder- en Jeugdpsychiatrisch centrum Wickraderheem (Mondriaan Zorggroep) en de nieuwe afdeling kinderpsychiatrie van het Academisch Ziekenhuis Maastricht. Sinds 1993 is hij als onderzoeker verbonden aan de Stichting Klinische Genetica Zuid Oost Nederland, Maastricht. Vanuit deze functie en onder leiding van prof.dr. J.-P. Fryns had hij de gelegenheid gehad onderzoek te verrichten op het vlak van gedrag bij genetische aandoeningen, wat tot dit proefschrift leidde. Sinds 2001 werkt hij terug als staflid bij de afdeling kinder- en jeugdpsychiatrie van het U.Z.Leuven.

Jean Steyaert is gehuwd met Charlotte Aerts, en de vader van Marie en Johanna. 


\section{Publicaties}

Steyaert J: Concentratie en leerstoornissen: diagnostiek en therapeutische implicaties vanuit een medischpedagogisch kader. (1990). In: International Reading Association, ed. Leesmoeilijkheden vamuit een neurologisch perspectief. Leuven.: ACCO.

Steyaert J, Pyck K. (1990). Beschouwingen bij de biologische aspecten van de aandachtsopbouw, Tydschrift wan de Vrije PMS-centra "Caleidscoop" vol. 3

Steyaert J, Van Damme-Lombaerts R. (1990). Coping of children in chronic haemodialysis and patterns of functioning of their families. Journal of the European Dialysis \& Thansplantation Nurses Association 18:24-26.

De Scheemaeker MJ, Volcke P. Steyact J. Fryns JP. (1992). Attention Deficit Hoperactivity Disorder: een multidisciplinaire nosologische benadering. Leuven: CME.

Steyaert J. (1992a). Recente trends in het onderzoek over aandachts- en hyperactiviteitstoornissen. Tydschrif voor Orthopedagogie, Kinderpsychiatrie en Kinderpsychologie 17

Steyaert J: Hoe erfelijk is dyslexie ? (1992b). In: Ruyssenaar A. ed. Dyslexie, nieune inzichten in onderzoek en behandeling. Leuven: ACCO.

Steyaen J, Borghgraef M, Gaulthier C. Fryns JP, Van den Berghe H. (1992). Cognitive profile in adult, normal intelligent female fragile X carriers. Am J Med Genet 43 (1-2):116-119.

Steyaert J. Borghgraef M. Fryns JP. (1994a). Apparently enhanced visual information processing in female fragile X carriers: preliminary findings. Am J Med Genet 51 (4):374-377.

Steyaert J, Decruyenaere M. Borghgraef M. Fryns JP. (1994b). Personality profile in adult female fragile X carriers: assessed with the Minnesota Multiphasic Personality Profile (MMPI). Am J Med Genet 51 (4):370-373.

Legius E, Descheemaeker MJ, Steyaer J, Spaepen A, Vlietinck R, Casaer P, Demaerel P, Fryns JP. (1995). Neurofibromatosis type 1 in childhood: correlation of MRI findings with intelligence. $J$ Neurol Neurosurg Psychiatry 59 (6):638-640.

Borghgraef M, Umans S, Steyaert J, Legius E, Fryns JP. (1996). New findings in the behavioral profile of young FraX females. Am J Med Genet 64 (2):346-349.

Steyaert J, Borghgraef M, Legius E, Fryns JP. (1996). Molecular-intelligence correlations in young fragile X males with a mild CGG repeat expansion in the FMR1 gene. Am J Med Genet 64 (2):274-277.

Swillen A, Hellemans H, Steyaert J, Fryns JP. (1996). Autism and genetics: high incidence of specific genetic syndromes in 21 autistic adolescents and adults living in two residential homes in Belgium. Am J Med Genet 67 (3):315-316.

Steyaert J, Umans S, Willekens D, Legius E, Pijkels E, de Die-Smulders C, Van den Berghe H, Fryns JP. (1997). A study of the cognitive and psychological profile in 16 children with congenital or juvenile myotonic dystrophy. Clin Genet 52 (3):135-141.

Fisch GS, Carpenter N, Holden JJ, Howard-Peebles PN, Maddalena A, Borghgraef M, Steyaert J, Fryns JP. (1999). Longitudinal changes in cognitive and adaptive behavior in fragile $\mathbf{X}$ females: a prospective multicenter analysis. Am J Med Genet 83 (4):308-312.

Goossens E, Steyaert J, De Die-Smulders C, Willekens D. Fryns JP. (2000). Emotional and behavioral profile and child psychiatric diagnosis in the childhood type of myotonic dystrophy. Genet Couns 11 (4):317. 327.

Steyaert J, de Die-Smulders C, Fryns JP, Goossens E, Willekens D. (2000). Behavioral phenotype in childhood type of dystrophia myotonica. Am J Med Genet 96 (6):888-889.

Kalff AC, Kroes M, Vles JS, Bosma H, Feron FJ, Hendriksen JG, Steyaert J, van Zeben TM, Crolla IF, Jolles J. (2001a). Factors affecting the relation between parental education as well as occupation and problem behaviour in Dutch 5- to 6-year-old children. Soc Psychiatry Psychiatr Epidemiol 36 (7):324-331.

Kalff AC, Kroes M, Vles JS, Hendriksen JG, Feron FJ, Steyaert J, van Zeben TM, Jolles J, van Os J. (2001b), Neighbourhood level and individual level SES effects on child problem behaviour: a multilevel analysis. J Epidemiol Community Health 55 (4):246-250.

Kroes M, Kalff AC, Kessels AG, Steyaert J, Feron FJ, van Someren AJ, Hurks PP, Hendriksen JG, van Zeben TM, Rozendaal N, Crolla IF, Troost J, Jolles J, Vles JS. (2001). Child psychiatric diagnoses in a population of Dutch schoolchildren aged 6 to 8 years. $J$ Am Acad Child Adolesc Psychiatry 40 (12): 1401-1409.

Kroes M, Kalff AC, Steyaert J, Kessels AG, Feron FJ, Hendriksen JG, van Zeben TM, Troost J, Jolles J, Vles JS. (2002). A longitudinal community study: do psychosocial risk factors and child behavior checklist scores at 5 years of age predict psychiatric diagnoses at a later age? $\mathrm{J} \mathrm{Am} \mathrm{Acad} \mathrm{Child} \mathrm{Adolesc}$ Psychiatry 41 (8):955-963. 
Steyaen J: The behavioural phenotype in Myotonic Dystrophy. (2002). In: Fisch GS, ed. Genetics and Genomics of Neurobehavioral Disorders, vol In Press. NY: Humana Press.

Steyaen J, Fryns JP. (2002). Review: Psychiatric Genetics: the case of single gene disorders. European Child and Adolescent Psychiatry In Press.

Steyaer J, Legius E, Borghgraef M, Fryns JP. (2002). A distinct neurocognitive phenotype in female fragile X premutation carriers asssessed with visual attention tasks. Am J Med Genet In Press 

